Aus der Klinik für Anästhesiologie

(Prof. Dr. med. M. Quintel)

der Medizinischen Fakultät der Universität Göttingen

\title{
ZENTRALE SENSIBILISIERUNG, SCHMERZLOKALISATION UND -AUSDEHNUNG BEI ARTHROSE DES HÜFTGELENKS
}

\author{
INAUGURAL-DISSERTATION \\ zur Erlangung des Doktorgrades \\ der Medizinischen Fakultät der \\ Georg-August-Universität zu Göttingen
}

vorgelegt von

\author{
Mathias Walter Siebertz \\ aus
}

Neuss am Rhein

Göttingen 2019 
Dekan:

Referent

Ko-Referent/in:

Drittreferent/in:
Prof. Dr. H. K. Kroemer

Prof. Dr. F. Petzke

Prof. Dr. M. Sommer

Prof. Dr. Th. Meyer

Datum der mündlichen Prüfung: 04.06.2020 
Hiermit erkläre ich, die Dissertation mit dem Titel "Zentrale Sensibilisierung, Schmerzlokalisation und -ausdehnung bei Arthrose des Hüftgelenks" eigenständig angefertigt und keine anderen als die von mir angegebenen Quellen und Hilfsmittel verwendet zu haben.

Göttingen, den 09.01.2019 
Publikation aus Teil-Ergebnissen dieser Arbeit:

Willett M, Siebertz M, Petzke F, Erlenwein J, Rushton A, Soldini E, Barbero M, Falla $D$ (2019): The extent of pain is associated with signs of central sensitization in patients with hip osteoarthritis. Pain Pract. 2020 Mar;20(3): 277-288. 


\section{Inhaltsverzeichnis}

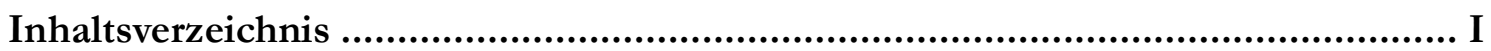

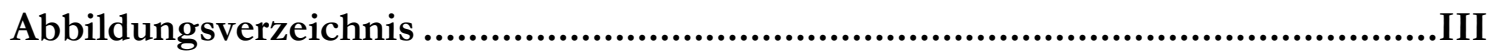

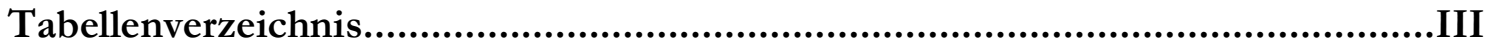

Abkürzungsverzeichnis .............................................................................

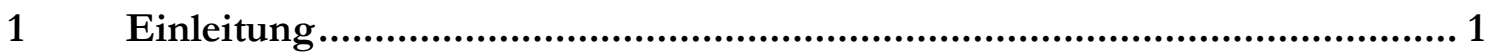

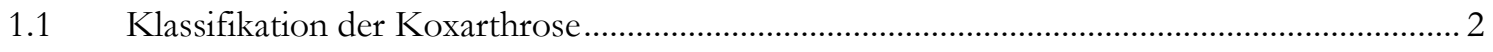

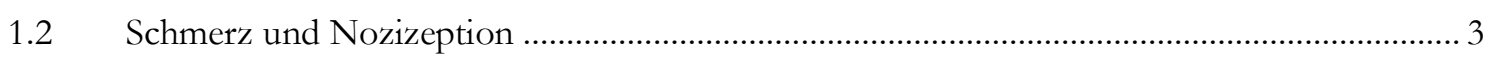

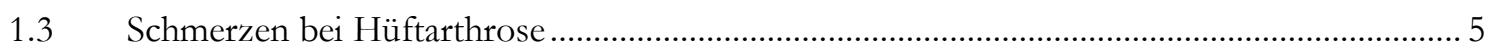

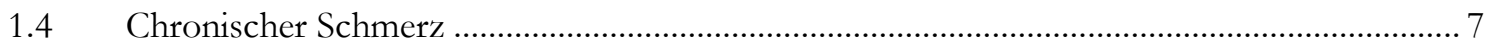

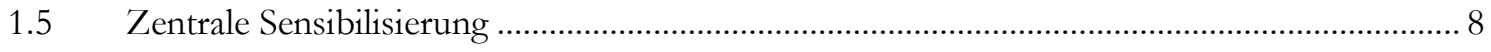

1.6 Klinische Kriterien der zentralen Sensibilisierung ............................................................. 9

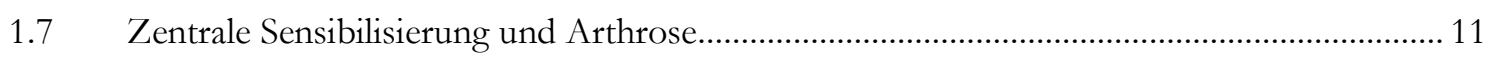

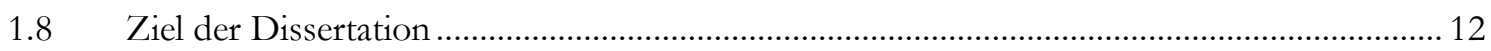

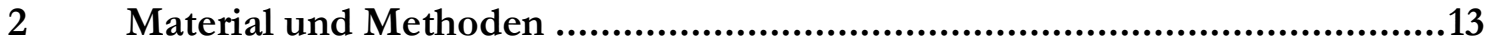

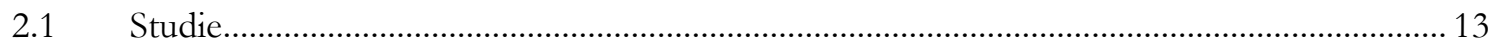

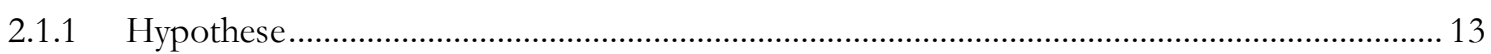

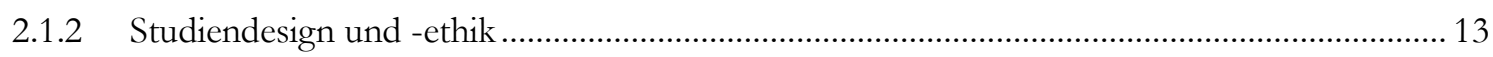

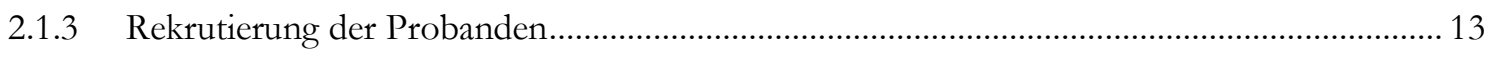

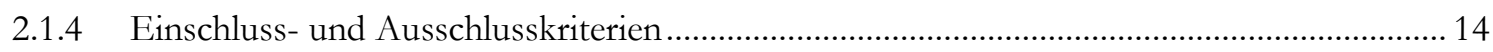

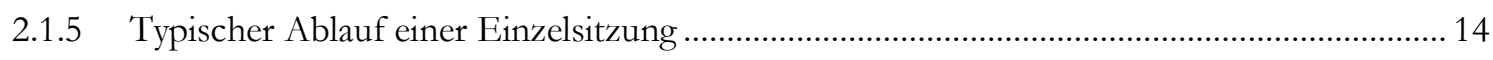

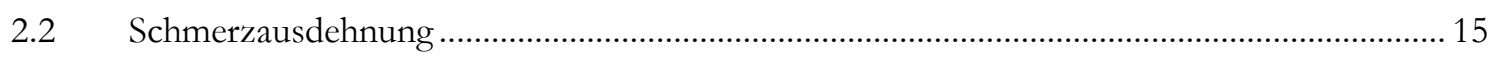

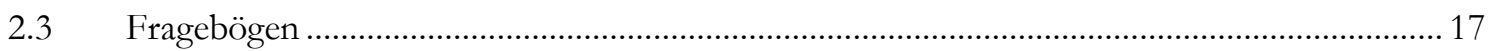

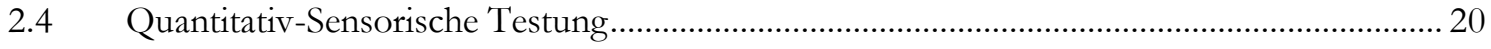

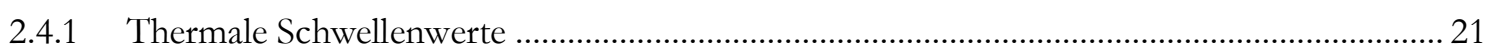

2.4.1.1 Cold Detection Threshold (CDT) und Warm Detection Threshold (WDT) ........................ 22

2.4.1.2 Cold Pain Threshold (CDT) und Heat Pain Threshold (HPT) ............................................. 22

2.4.2 Druckschmerzschwellen/ Pressure Pain Threshold (PPT) ………….................................. 24

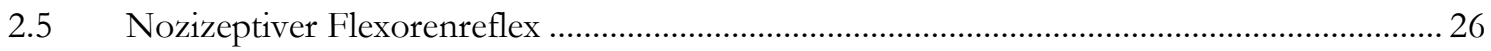

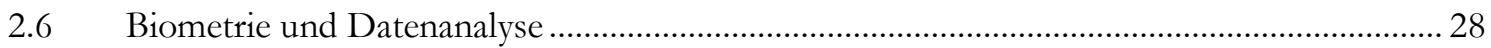

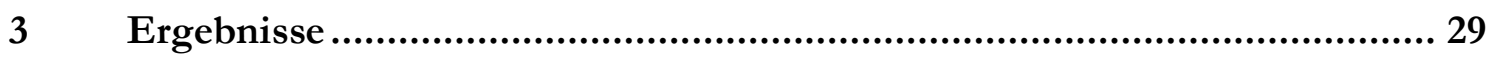

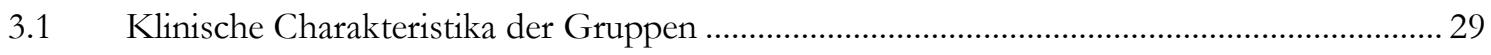

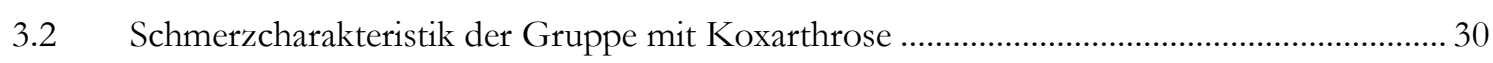

3.3 Ergebnisse des Fibromyalgie-Syndrom Survey Questionnaires ............................................ 33 


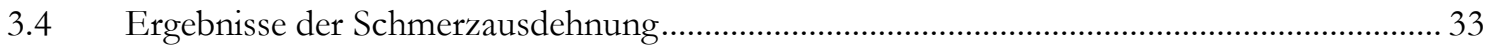

3.5 Ergebnisse der psychologischen Fragebögen .............................................................. 35

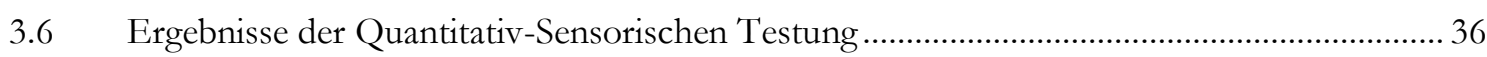

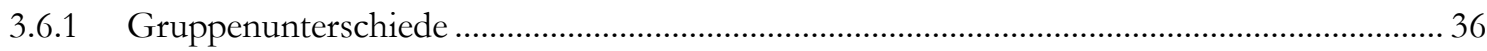

3.6.2 Unterschiede zwischen betroffener und nicht betroffener Seite ........................................ 38

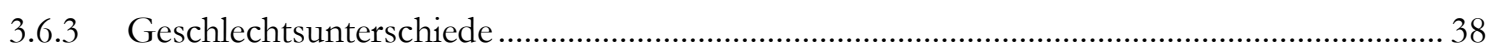

3.7 Korrelationen zwischen verschiedenen Mess-Parametern.................................................. 42

3.7.1 Korrelationen zwischen der Schmerzausdehnung und Ergebnissen der Fragebögen .......... 42

3.7.2 Korrelationen zwischen der Schmerzausdehnung und Ergebnissen der QST .................... 42

3.7.3 Korrelationen zwischen der Druckschmerzempfindlichkeit und weiteren Variablen ..........42

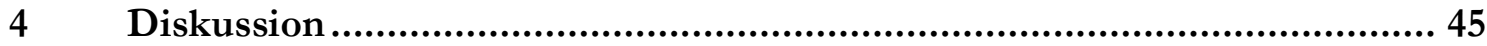

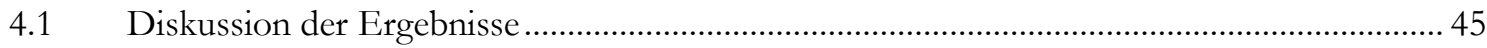

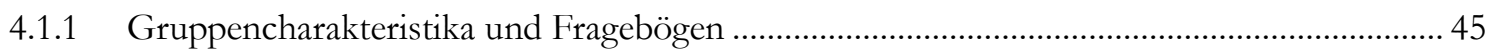

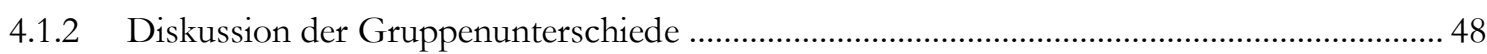

4.1.3 Prüfung der klinischen Kriterien für zentrale Sensibilisierung ......................................... 49

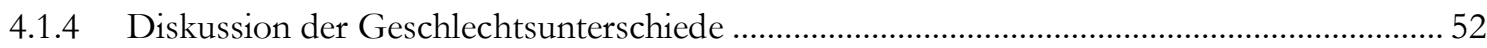

Einschränkungen und Stärken der Arbeit .................................................................. 53

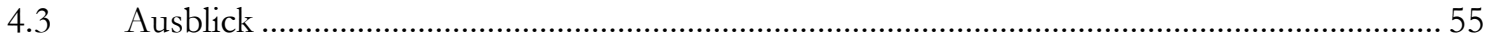

$5 \quad$ Zusammenfassung........................................................................ 58

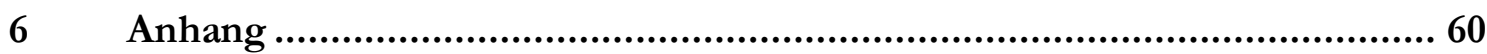

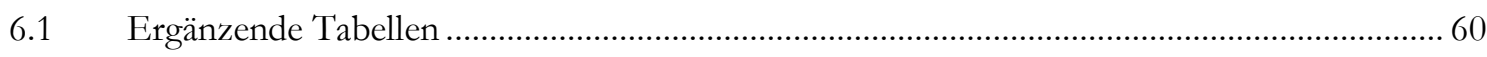

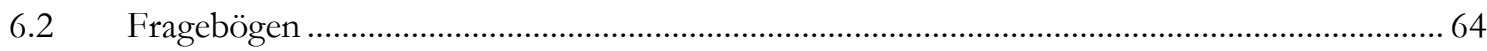

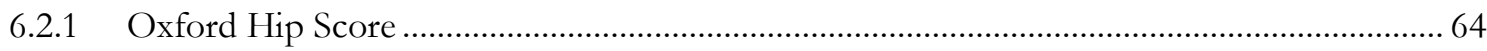

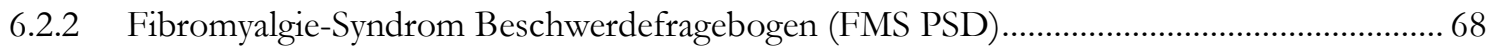

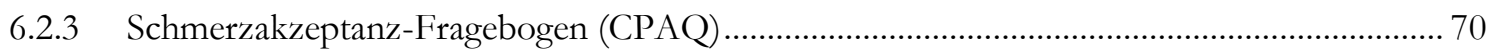

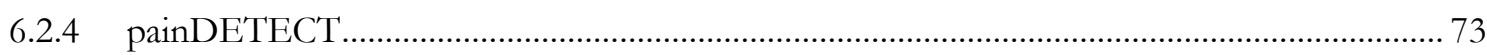

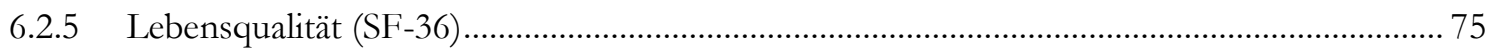

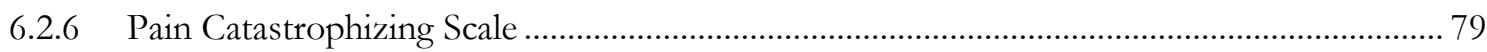

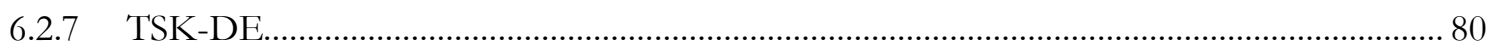

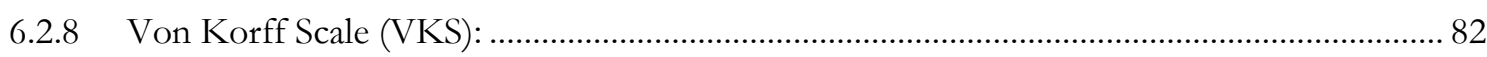

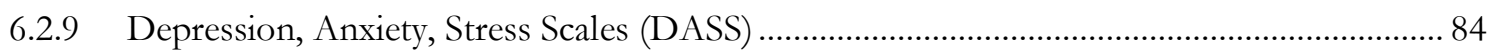

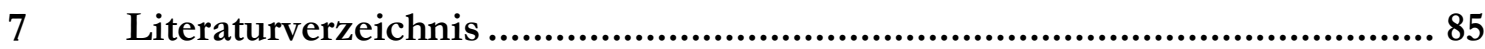




\section{Abbildungsverzeichnis}

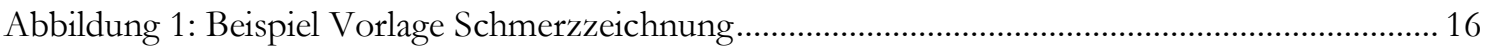

Abbildung 2: Beispiele der vorgefertigten Schablonen für beide Geschlechter .................................. 16

Abbildung 3: Übersicht über die verschiedenen Untersuchungspunkte von ventral........................... 21

Abbildung 4: Der Thermal Sensory Analyzer TSA-II, Firma Medoc ................................................. 23

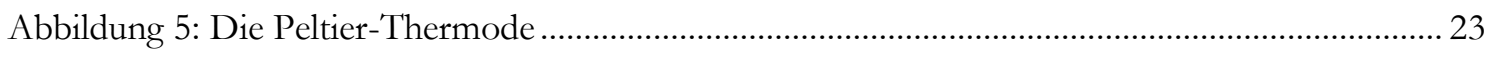

Abbildung 6: Das Algometer der Firma Somedic, Sweden ............................................................. 25

Abbildung 7: Position der Reiz- und Messelektroden beispielhaft am linken Bein ............................ 27

Abbildung 8: Display des Paintracker vor Beginn einer Messung....................................................... 27

Abbildung 9: Zeitliches Schmerzmuster der Patienten mit Koxarthrose ............................................. 30

Abbildung 10: Verteilung des Oxford Hip Scores innerhalb der Hüftarthrosegruppe ........................ 31

Abbildung 11: Verteilung der Probanden mit Hüftarthrose auf die Von-Korff-Grade..................... 32

Abbildung 12: Ergebnisse der Schmerzkarten bei weiblichen Probanden ........................................... 34

Abbildung 13: Ergebnisse der Schmerzkarten bei männlichen Probanden............................................ 34

Abbildung 14: Hüftgelenksferne Druckschmerzschwellen .................................................................. 37

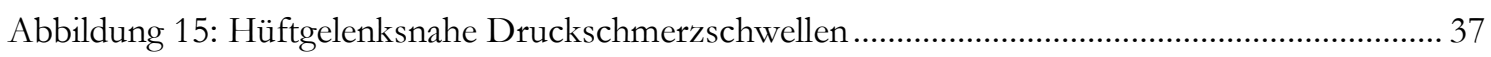

Abbildung 16: Hüftgelenksferne Druckschmerzschwellen im Vergleich zwischen Frauen und Männern innerhalb der Hüftarthrosegruppe ......................................................................... 40

Abbildung 17: Hüftgelenksnahe Druckschmerzschwellen im Vergleich zwischen Frauen und Männern innerhalb der Hüftarthrosegruppe .......................................................................... 40

Abbildung 18: Wärmedetektions- (WDT) und -Wärmeschmerzschwellen (HPT) innerhalb der Gruppe mit Hüftarthrose .................................................................................................. 41

Abbildung 19: Kältedetektions- (CDT) und Kälteschmerzschwellen (CPT) innerhalb der Gruppe

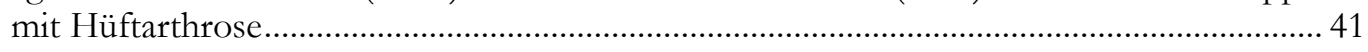

Abbildung 20: Korrelation zwischen Oxford Hip Score und hüftgelenksnahem PPT ....................... 43

Abbildung 21: Korrelation zwischen Oxford Hip Score und hüftgelenksfernem PPT ..................... 43

\section{Tabellenverzeichnis}

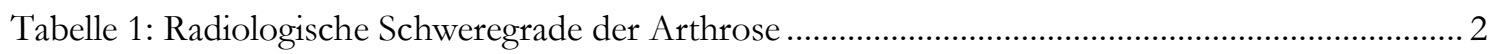

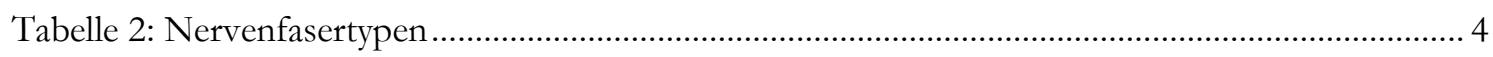

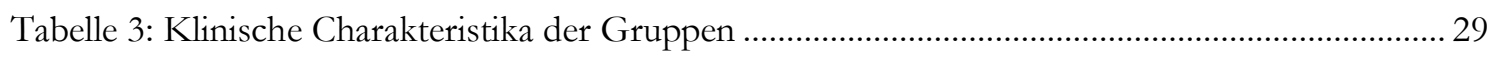

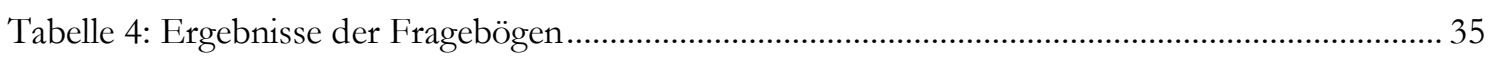

Tabelle 5: Übersicht der statistisch signifikanten QST-Gruppenunterschiede..................................... 36

Tabelle 6: Signifikante Korrelationen des Body Mass Index mit Variablen der QST vor und mit

Korrektur für den Faktor Geschlecht ................................................................................... 44

Tabelle 7: QST-Ergebnisse für betroffene und nicht betroffene Seite innerhalb der Gruppe mit

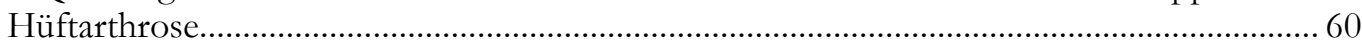

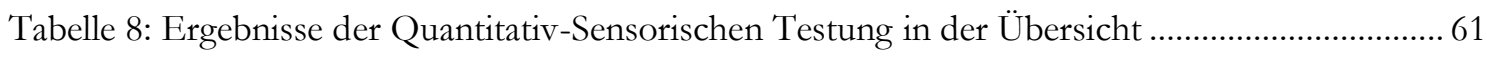

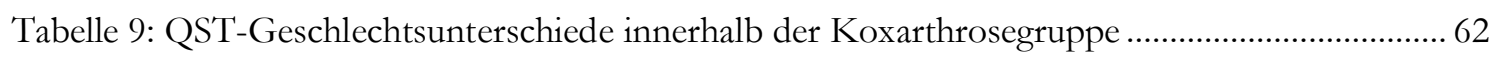

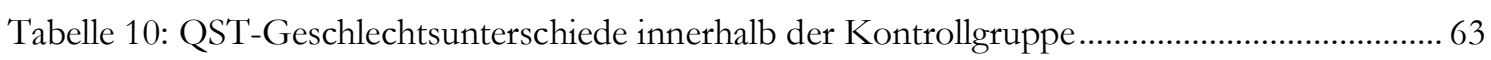




\section{Abkürzungsverzeichnis}

ACR

ANOVA

BMI

CDT

CPT

CPAQ

CSI

DASS

DFNS

FMS

HPT

ICD-(10), 11

OHS

PCS

PPT

QST

RA

SF-36

SSS

TSK

VKS

WDT

WDR-Neurone

WPI

WOMAC

ZNS
American College of Rheumatology

Analysis of variance

Body Mass Index

Cold Detection Threshold

Cold Pain Threshold

Chronic Pain Acceptance Questionnaire

Central Sensitization Inventory

Depression, Anxiety, Stress Scale

Deutscher Forschungsverband neuropathischer Schmerz

Fibromyalgie Syndrom

Heat Pain Threshold

International Statistical Classification of Diseases and Related Health

Problems, Version (10), 11

Lendenwirbelsäule

Oxford Hip Score

Pain Catastrophizing Scale

Pressure Pain Threshold

Quantitativ-Sensorische Testung

Rheumatoide Arthritis

Short Form-36 Health (Related Quality of Life) Survey

Symptom Severity Score (Fibromyalgie-Syndrom Survey-Questionnaire)

Tampa Scale for Kinesiophobia

Von Korff Scale

Warm Detection Threshold

Wide Dynamic Range Neurone

Widespread Pain Index (Fibromyalgie-Syndrom Survey-Questionnaire)

Western Ontario \& McMaster Universities Osteoarthritis Index

Zentrales Nervensystem 


\section{Einleitung}

Die Osteoarthrose des Hüftgelenks (Koxarthrose) stellt im Kontext der zunehmenden Alterung der Gesellschaft eine wichtige medizinische und sozioökonomische Herausforderung dar. Neben hohen direkten Kosten für das Gesundheitssystem durch die Inanspruchnahme von diagnostischen, kurativen und rehabilitativen Leistungen entstehen auch indirekte Kosten durch reduzierte berufliche Leistungsfähigkeit und persönliche Einbußen in der Lebensqualität (Dunlop et al. 2003). Während epidemiologische Daten zur Verbreitung der Arthrose deutschlandweit stark variieren, konnte gezeigt werden, dass die Erkrankung zu den weltweit häufigsten Gelenkerkrankungen zählt (Hootman und Helmick 2006) und im Rahmen der demographischen Entwicklung zunehmend weiter an Relevanz gewinnen wird (Rabenberg 2013). Zwar wird die Erkrankung häufiger bei den Älteren unserer Gesellschaft diagnostiziert, doch weisen Studien darauf hin, dass durchaus auch jüngere Menschen betroffen sein können (Harris und Coggon 2015). Hierdurch kann es neben relevanten Einbußen der Arbeitskraft bei Erwerbstätigen auch zu Limitierungen der Lebenszufriedenheit im Alter kommen. Bezüglich der Verteilung auf die beiden Geschlechter finden sich in den meisten Untersuchungen insgesamt mehr betroffene Frauen als Männer (Quintana et al. 2008, Cross et al. 2014).

Definiert ist die Arthrose der Hüfte als degenerative Erkrankung des Hüftgelenks, welche initial vorzugsweise den Gelenkknorpel betrifft. Weiterhin charakterisiert wird sie durch „Schmerzen und Funktionsstörungen (...)“, die „durch die degenerative Zerstörung des Gelenkknorpels und der Schädigung angrenzender Strukturen wie Knochen, Muskeln, Kapseln und Bänder“ (Rabenberg 2013, S. 7) pathophysiologisch erklärt werden können. Durch den lokalen Verschleiß der Gelenk-Binnenstrukturen und inkongruente Gelenkflächen kann der Knorpel innerhalb des betroffenen Gelenks seiner Funktion als mechanischer Stoßdämpfer häufig nicht mehr adäquat nachkommen. Da dem Gelenk physiologisch die Fähigkeit fehlt, Knorpel suffizient nachzubilden, gab es zuletzt auch immer wieder Versuche, über die Transplantation von Knorpel eine Heilung zu erreichen (Bobic 1999, Fontana et al. 2012). Bislang fehlt es dieser bislang noch eher experimentellen Option für eine regelmäßige klinische Anwendung allerdings an prospektiven Langzeitstudien. Stattdessen stehen therapeutisch neben operativen Interventionen konservativ vor allem Allgemeinmaßnahmen wie Gewichtsreduktion, physikalische Therapie (Peeters et al. 2017) und medikamentöse Supportivmaßnahmen (Neogi et al. 2018) im Vordergrund. 
Wichtig ist allerdings, die Arthrose nicht nur als Erkrankung des Knorpels zu verstehen, sondern vielmehr als eine Einschränkung, die das gesamte Gelenk und seine Strukturen betrifft (Loeser et al. 2012). Während bei Ursachen für die primären Formen der Arthrose am ehesten auf genetische Faktoren verwiesen wird, ist die Genese der sekundären Arthrose häufig eng mit den Faktoren Übergewicht, Traumata, berufliche Überbelastung, Gelenkfehlstellungen und biomechanische Instabilität verknüpft (Rehart und Lehnert 2008). Auch eine metabolische, endokrinologische oder posttraumatische Ätiologie werden beschrieben (Rabenberg 2013).

\subsection{Klassifikation der Koxarthrose}

Es existieren zahlreiche funktionelle Klassifikationen zur Einteilung der Hüftarthrose wie beispielsweise der Harris Hip Score, der Score nach Merle d'Aubigne (D’Aubigne und Postel 1954) oder der WOMAC-Score (Stucki et al. 1996). Mit diesen werden häufig Aspekte wie Schmerz, Steifheit sowie körperliche Funktion bzw. körperliche Einschränkungen in verschiedenen Alltagssituationen evaluiert. Nach den Kriterien des American College of Rheumatology (ACR) wird anhand verschiedener klinischer Symptome wie beispielsweise einer eingeschränkten Innenrotation des Hüftgelenks oder Schmerzen bei Innenrotationsbewegungen in der Anamnese oder der körperlichen Untersuchung die Diagnose Koxarthrose gestellt. Zusätzlich gehen Laborwerte wie beispielsweise eine erhöhte Blutsenkungsgeschwindigkeit oder Röntgenbilder in die Diagnosefindung mit ein (Altman et al. 1991).

Klinisch wird die (Hüft)Arthrose häufig anhand eines Röntgenbildes nach der Kellgren and Lawrence-Klassifikation eingestuft. In dieses Schema (Tabelle 1) gehen lediglich röntgenologische Auffälligkeiten wie beispielsweise Gelenkspaltveränderungen oder Osteophyten, also Knochenneubildungen, die keine Gelenkfunktion haben, mit ein (Kellgren und Lawrence 1957).

\begin{tabular}{llll}
\hline Grad I & Grad II & Grad III & Grad IV \\
\hline $\begin{array}{l}\text { geringfügige } \\
\text { subchondrale } \\
\text { Sklerosierungen }\end{array}$ & $\begin{array}{l}\text { beginnende } \\
\text { Verschmälerung des } \\
\text { Gelenkspaltes }\end{array}$ & $\begin{array}{l}\text { deutlich inkongruente } \\
\text { Gelenkfläche }\end{array}$ & $\begin{array}{l}\text { vollständige } \\
\text { Gelenkdestruktion }\end{array}$ \\
keine Osteophyten & Osteophyten & Osteophyten & ggf. Nekrosen \\
$\begin{array}{l}\text { keine Veränderungen } \\
\text { am Gelenkspalt }\end{array}$ & & kräftige & \\
& & Verschmälerung des & \\
& & Gelenkspaltes & \\
\hline
\end{tabular}

Tabelle 1: Radiologische Schweregrade der Arthrose, modifiziert nach Kellgren und Lawrence 1957 
So häufig diese radiologische Klassifikation zur Diagnostik im klinischen Alltag auch verwendet wird, für die Schweregradeinteilung der Schmerzen von Betroffenen mit Koxarthrose ist sie nicht unbedingt geeignet. Es konnte vielmehr gezeigt werden, dass Diskordanzen zwischen dem Grad der Destruktion des Gelenkknorpels und der geklagten Schmerzintensität bei Patienten existieren (Finan et al. 2013, Kim et al. 2015). So ist keinesfalls ein höherer Grad der Gelenkzerstörung auch automatisch mit stärkeren Schmerzen assoziiert.

\subsection{Schmerz und Nozizeption}

Schmerz wird als „ein unangenehmes Sinnes- und Gefühlserlebnis, das mit aktueller oder potentieller Gewebsschädigung verknüpft ist“ (IASP 2015, S.1), definiert. Davon abzugrenzen ist der Begriff der Nozizeption.

In der Physiologie beschreibt Nozizeption die neuronalen Prozesse der Kodierung und Verarbeitung noxischer Reize, die durch die Präsenz von freien Nervenendigungen im Gewebe, in der Haut und anderen Organen gewährleistet werden. Nozizeptoren entsprechen den ersten Neuronen im schmerzverarbeitenden System, deren Zellkörper entweder im Spinalganglion oder in den sensiblen Hirnnervenkernen liegen (Grafe 2008). Über unterschiedliche Mechanismen der Transduktion, also der Umwandlung eines Reizes in ein körpereigenes Signal (Meßlinger 2010), wird das Neuron aktiviert. So werden in dem Moment einer Gewebszerstörung oder -schädigung durch thermische, mechanische oder chemische Noxen eine Vielzahl von Botenstoffe wie beispielsweise Bradykinin, Histamin oder Prostaglandin, aber auch $\mathrm{H}+-_{+}$, und Kalium-Ionen aus geschädigten Zellen, freigesetzt. Über Öffnung von Ionenkanälen und intrazelluläre Signalkaskaden führt dies $\mathrm{zu}$ einer Bildung von Rezeptorpotenzialen an den Nozizeptoren. Diese Signale werden in ein Aktionspotenzial umgewandelt und über nozizeptive Neurone, nicht myelinisierte (C-Fasern) oder nur dünn myelinisierte Axone (A $\delta$-Fasern) weiter ins Rückenmark geleitet, um dort das sensible Hinterhorn zu erreichen. Während nach der Einteilung von (Erlanger und) Gasser (Tabelle 2) über die A $\delta$-Fasern vornehmlich ein gut lokalisierbarer Schmerz sowie mechanozeptive Informationen übertragen werden, leiten die C-Fasern primär thermische Informationen und den sogenannten ,zweiten Schmerz“.

Dieser ist durch einen eher dumpfen und nur schlecht lokalisierbaren Charakter bekannt. AßFasern hingegen scheinen über die Verbindung mit hemmenden Interneuronen eine Beteiligung an der segmentalen endogenen Schmerzhemmung auf Rückenmarksebene zu haben (Melzack und Wall 1965), leiten selbst aber keine spezifisch noxischen Signale. 
Nach einer ersten synaptischen Umschaltung im Hinterhorn des Rückenmarks steigen die Informationen in den Vorderseitensträngen (Tractus spinothalamicus anterior und Tractus spinothalamicus lateralis) über Kerne des Thalamus in Richtung kortikaler Areale.

Über die Projektion in die Formatio reticularis werden die autonom-vegetativen Komponenten einer Schmerzerfahrung erklärt. Typische begleitende Stressreaktionen wie beispielsweise eine Tachykardie oder verstärkte Transpiration können somit verstanden werden (Traue et al. 2013). Affektiv-emotionale Bewertungen werden durch die Projektion ins limbische System, kognitive Bewertungen unter anderem über die Verbindung in den präfrontalen Kortex vorgenommen (Loggia et al. 2015).

\begin{tabular}{|c|c|c|c|c|c|}
\hline $\begin{array}{l}\text { Erlanger \& } \\
\text { Gasser }\end{array}$ & $\begin{array}{l}\text { Lloyd \& } \\
\text { Hunt }\end{array}$ & $\begin{array}{l}\text { Myelisierungs- } \\
\text { grad }\end{array}$ & $\begin{array}{l}\text { Durch- } \\
\text { messer } \\
(\mu \mathrm{m})\end{array}$ & $\begin{array}{l}\text { Leitungsge- } \\
\text { schwindigkeit } \\
(\mathrm{m} / \mathrm{s})\end{array}$ & Vorkommen \\
\hline $\mathrm{A} \alpha$ & I & ++ & $10-20$ & $60-120$ & $\begin{array}{l}\text { Muskelspindelafferenzen, } \\
\alpha \text {-Motoneurone }\end{array}$ \\
\hline$A B$ & II & + & $7-15$ & $30-70$ & Mechanoafferenzen der Haut \\
\hline $\mathrm{A} \gamma$ & & + & $4-8$ & $15-50$ & Muskelspindelefferenzen \\
\hline A $\delta$ & III & + & $2-7$ & $10-40$ & $\begin{array}{l}\text { Thermoafferenzen, } \\
\text { "heller Sofortschmerz" }\end{array}$ \\
\hline B & & + & $1-3$ & $3-20$ & präganglionäre vegetative Fasern \\
\hline $\mathrm{C}$ & IV & marklos & $0,5-1,5$ & $0,5-2$ & $\begin{array}{l}\text { "dumpfer Spätschmerz", } \\
\text { postganglionäre vegetative Fasern }\end{array}$ \\
\hline
\end{tabular}

Tabelle 2: Nervenfasertypen modifiziert nach Gasser 1941

Unter der endogenen Schmerzhemmung wird im Allgemeinen ein hochkomplexes System aus verschiedenen Bahnen verstanden, die zentral von den Raphe-Kernen, dem periaquäduktalen Grau und dem Locus coeruleus ausgehen und vornehmlich auf Rückenmarksebene einkommende nozizeptive Informationen über noradrenerge oder serotonerge Verschaltungen regulieren können. Dies ist entwicklungsgeschichtlich am ehesten durch die Notwendigkeit zu erklären, den Organismus auch bei stärksten Schmerzen zunächst aus unmittelbarer Lebensgefahr zu bringen (Locher et al. 2011).

Im Rückenmark gibt es neben den spezifischen nozizeptiven Neuronen, die ausschließlich Synapsen mit entsprechenden C-Fasern oder A $\delta$-Fasern ausbilden, sogenannte Wide-Dynamic Range-Neurone (WDR), die synaptisch mit allen Nervenfasertypen der norizeptiven Bahn verbunden sind. Diese WDR-Neurone werden somit nicht ausschließlich durch die typischen Noxen aktiviert, sondern vielmehr durch die gesamte Bandbreite der sensorischen Stimuli, beispielsweise auch durch leichte Berührungen (D’Mello und Dickenson 2008). Der Nachweis 
solcher Neurone erleichtert das Verständnis typischer Phänomene bei chronischen Schmerzen wie zum Beispiel der Allodynie und Hyperalgesie, auf die im Verlauf weiter eingegangen werden wird.

\subsection{Schmerzen bei Hüftarthrose}

Neben einer massiven Limitierung der Lebensqualität durch Einschränkung der Funktion bei Patienten mit Arthrose (Jinks et al. 2007) ist häufig vor allem der assoziierte Schmerz krankheitsbestimmend. Typischerweise klagen Patienten mit Arthrose über progrediente Schmerzen bei Belastung und weisen einen sogenannten Anlaufschmerz auf. Dieser äußert sich zu Beginn einer Bewegung aus einer Ruhestellung heraus nach beispielsweise längerem Sitzen. Menschen mit Hüftgelenksarthrose erleben typischerweise Schmerzen im Bereich des Gesäßes, der Leiste und des Oberschenkels bis zum Knie (Khan et al. 2004). Im Bereich des Musculus gluteus medius (Amaro et al. 2007), des Trochanter major und des lateralen Oberschenkels (Kosek und Ordeberg 2000b) lässt sich häufig ein maximaler Schmerz beobachten. Das klassische Verständnis von Schmerzen bei Arthrose ist ein primär mechanisches, das durch die Reizung peripherer Nozizeptoren in der Gelenkkapsel und vor allem im vorderen Synovium (Dye et al. 1998 ) entsteht. Der Knorpel selbst ist ein bradytrophes Gewebe und primär nicht schmerzempfindlich. Aufgrund der Irritation innerhalb des Gelenkes und der veränderten Biomechanik scheint ein solch rein mechanisches Schmerzerleben plausibel. Über die Jahre wurden zahlreiche weiterführende Theorien veröffentlicht, die Schmerzen bei Arthrose unter anderem durch eine Beteiligung von Nervenanteilen des Periosts (Grönblad et al. 1984), chronischen Entzündungen der Synovia (Smith et al. 1997), Läsionen des Knochenmarks (Felson et al. 2001) oder erhöhtem intraossärem Druck nahe geschädigter Gelenke (Arnoldi et al. 1980), erklären sollten.

Allerdings konnte auch gezeigt werden, dass sich Schmerz gleichfalls ohne fortwährenden nozizeptiven Input dauerhaft präsentieren und damit chronifizieren kann (Treede 2013b). Weiterhin hat die hohe interindividuelle Schwankung der Symptome und häufige Diskrepanz zwischen dem Grad des strukturellen Schadens im Gelenk und der damit verbundenen Schmerzintensität dazu geführt, dass der Verdacht auf eine zentrale Komponente der Schmerzen bei Arthrose geäußert wurde (Lluch Girbes et al. 2013). So scheint der ständige Input von peripheren Nozizeptoren der Gelenke auf Neurone des sensiblen Hinterhorns zu einem Umbau zentraler neuronaler Verbindungen beizutragen (Lluch Girbes et al. 2013). Hierzu passt, dass in Tierversuchen nachgewiesen werden konnte, dass strukturell-arthrotische Veränderungen des Kniegelenks im Verlauf auch zu zentralen Umbauprozessen des Rückenmarks führen können (Im et al. 2010). 
In Kapitel 1.7 wird genauer auf den Kenntnisstand zu zentralen Veränderungen der Schmerzverbreitung bei Osteoarthose der Hüfte eingegangen. 


\subsection{Chronischer Schmerz}

Im Vergleich zu akutem Schmerz fehlt bei der chronischen Form eine einheitliche Definition. Häufig wird chronischer Schmerz über die Dauer definiert (typischerweise $>3$ Monate) oder erst bei Beschwerdepersistenz als solcher bezeichnet (Treede et al. 2015). Aktuell wird eine Vereinheitlichung u.a. der erhöhten generalisierten Schmerzempfindung bei chronischen Schmerzen im Rahmen der Novellierung der internationalen Klassifikation der Krankheiten (ICD-11) angestrebt (Butler et al. 2016). Dies soll vor allem eine verbesserte Vergleichbarkeit zwischen neu gewonnenen Studienerkenntnissen in Zukunft gewährleisten (Erlenwein et al. 2017).

Bei der Chronifizierung von Schmerzen tritt auch der Zusammenhang zwischen einer Warnund Hinweisfunktion und der Schmerzempfindung in den Hintergrund (Von Korff und Miglioretti 2005).

Obwohl die Prävalenz chronischer Schmerzen (von mehr als drei Monaten Dauer) in Deutschland auf etwa $27 \%$ geschätzt wird (Häuser et al. 2015) und damit durchaus als häufig bezeichnet werden kann, bleibt das pathophysiologische Verständnis der Zusammenhänge einer chronifizierten Schmerzerkrankung weiter verbesserungsbedürftig. So liegt in der Studie von Häuser die Häufigkeit chronischer Schmerzen mit starker Einschränkung der Funktion bei 7,4 $\%$, bei noch zusätzlicher psychischer Symptomatik bei 2,8 \%. Auch weltweit wird die Prävalenz aller chronischer Schmerzerkrankungen zusammengefasst auf etwa $20 \%$ geschätzt (Breivik et al. 2006, Gureje et al. 2008).

Diese heterogene Gruppe besteht neben chronischen muskuloskelettalen Schmerzen unter anderem auch aus chronisch tumorassoziierten, chronisch neuropathischen und chronisch posttraumatischen bzw. postinterventionellen Schmerzen. Immer häufiger wurden zuletzt auch Klassifikationen geschaffen, die Schmerzen bei Patienten in Kategorien wie nozizeptiv, peripher neuropathisch und mit zentraler Beteiligung einteilen (Smart et al. 2010a) und die im Kontext degenerativer Erkrankungen aus dem muskuloskelettalen Formenkreis wie dem Kreuzschmerz validiert wurden (Smart et al. 2010b). Inwieweit diese Erkenntnisse auf andere Erkrankungen wie die Arthrose übertragbar sind, bleibt bislang unbekannt.

Sicher dagegen ist, dass Patienten mit chronischen Schmerzen präoperativ ein erhöhtes Risiko für postoperative Schmerzexazerbationen haben (Gerbershagen et al. 2014, Lavand'homme 2017). Für zahlreiche operative Eingriffe konnte zudem gezeigt werden, dass die Entwicklung von postoperativ persistierenden chronischen Schmerzen keine Seltenheit ist (Perkins und Kehlet 2000, Erlenwein et al. 2017). Dies unterstreicht wie wichtig es ist, verbesserte Methoden zu entwickeln, um Anzeichen für bestehenden chronischen Schmerz oder einem erhöhten 
Risiko für eine Chronifizierung beispielsweise vor Operationen besser zu erkennen und präventiv rechtzeitig entgegenwirken zu können.

Studien zeigen, dass frühe klinische Warnhinweise auf einen derart chronischen Verlauf von Schmerzen existieren und Patienten Zeichen einer sogenannten zentralen Sensibilisierung zeigen können (Mease et al. 2011, Suokas et al. 2012).

\subsection{Zentrale Sensibilisierung}

Die zentrale Sensibilisierung wird als potentiell reversibler Anstieg der synaptischen Effizienz und Erregbarkeit von Neuronen in nozizeptiven Bahnen des zentralen Nervensystems (ZNS) bezeichnet (Woolf 2011). Der ursächliche neuronale Umbau und die daraus resultierende veränderte strukturelle Plastizität des ZNS wird durch zahlreiche pathophysiologische Theorien erklärt: Unter anderem konnten veränderte spinale Reflexe (Emery et al. 2006; Courtney et al., 2009), auffällig veränderte proinflammatorische Zytokinspiegel (Lee et al. 2011), schmerzspezifische Lern- und Gedächtnisleistungen (Pfingsten et al. 2015) und andere psychologische Kofaktoren (Bennell et al. 2015) im Zusammenhang mit dem Auftreten von zentraler Sensibilisierung nachgewiesen werden. Auch dysfunktionale körpereigene analgetische Mechanismen (Meeus et al. 2008) und Phänomene wie die Langzeitpotenzierung (Sandkühler 2000) werden als mögliche Erklärungsansätze gesehen. In den meisten Fällen resultiert, womöglich vermittelt durch die bereits oben beschriebenen WDR-Neurone, ein verändertes Schmerzempfinden. Dieses ist insbesondere durch Phänomene wie Hyperalgesie und Allodynie charakterisiert. Während die Hyperalgesie als unverhältnismäßig starke Schmerzreaktion auf typische Schmerzreize (zum Beispiel Nadelstiche) beziehungsweise Verletzungen bezeichnet wird, ist die Allodynie als Schmerzerleben auf per se eigentlich nicht schmerzhafte Reize zu sehen, wie beispielsweise einfache Berührung.

Weiterhin kann aus einer zentralen Sensibilisierung ein objektiv diskrepantes Verhältnis zwischen Schmerzempfinden und nachvollziehbar geschädigtem Gewebe resultieren.

Trotz der Fülle an möglichen Mechanismen und Erklärungsansätzen bleibt der Großteil der komplexen Zusammenhänge dieses sehr heterogenen Phänomens unverstanden und ist daher weiterhin Gegenstand aktueller Forschung.

Unklar ist auch die Frage der Quantifizierung und Erfassung im klinischen Kontext. Nachgewiesen ist, dass Mechanismen der zentralen Sensibilisierung Einfluss auf Schmerzentstehung und -erleben chronischer Schmerzerkrankungen nehmen können, wie bei chronischen Schmerzen im Bereich der Lendenwirbelsäule (LWS) (Sanzarello et al. 2016), der rheumatoiden Arthritis und dem Fibromyalgiesyndrom (Gracely et al. 2002; Petzke et al. 2005; Staud 2011). 
Auch für Arthrose konnten ähnliche Zusammenhänge bereits nachgewiesen werden (Nijs et al. 2014). Leider mangelt es darüber hinaus im klinischen Alltag an Möglichkeiten der strukturierten Erfassung von Anzeichen zentraler Sensibilisierung bei Patienten mit muskuloskelettalen, aber auch anderen Schmerzerkrankungen. Neben Versuchen, mithilfe bildgebender Verfahren (Giesecke et al. 2005, Parks et al. 2011) oder richtungsweisender laborchemischer Marker (Arendt-Nielsen et al. 2014) Einblicke in die Ursachen zentraler Sensibilisierung bei chronischen Schmerzerkrankungen zu erhalten, wurden vor geraumer Zeit auch klinische Kriterien vorgeschlagen, die die Klassifikation einer zentralen Sensibilisierung vereinfachen könnten (Nijs et al. 2014).

\subsection{Klinische Kriterien der zentralen Sensibilisierung}

Anhand klinischer Kriterien werden die oben beschriebenen Phänomene wie Allodynie, Hyperalgesie und im Ausmaß zur nachvollziehbaren Schädigung unverhältnismäßig erscheinende Schmerzen systematisiert und durch zusätzliche Fragebögen wie dem Central Sensitization Inventory (CSI) ergänzt. Hierdurch werden sowohl somatische Symptome wie beispielsweise Schlafstörungen, als auch emotionale Symptome erfasst (Mayer et al. 2012, Cuesta-Vargas et al. 2018). Der entsprechende Fragebogen (CSI) wird derzeit erst in deutscher Übersetzung validiert.

Bisher sind diese Kriterien allerdings in der klinischen Anwendung und Erfahrung kaum validiert und etabliert. Das Ziel unserer Studie a priori war zwar nicht direkt die Kriterien von Nijs et al. 2014 auf klinische Anwendbarkeit zu prüfen, allerdings kann anhand dieser Kriterien eine systematische Herangehensweise an das komplexe Thema der zentralen Sensibilisierung bei Menschen mit einer chronisch-degenerativen muskuloskelettalen Schmerzerkrankung erfolgen. Daher wird im Verlauf dieser Arbeit an verschiedenen Stellen Bezug auf die in der Folge beschriebenen Kriterien genommen.

Einschränkend postulieren die Autoren das Vorliegen neuropathischer Schmerzen, also Schmerzen, die durch „mechanische, metabolische, toxische und entzündliche Einwirkungen“ (Binder et al. 2013, S. 364) direkt auf sensible Nervenzellen und damit deren Schädigung entstehen, zunächst im Vorfeld auszuschließen.

Im Anschluss werden drei Kriterien beschrieben, die helfen sollen klinische Anzeichen einer zentralen Sensibilisierung systematisch zu erkennen (Nijs et al. 2014). 


\section{Kriterium 1 ("Disproportion of pain to the nature and extent of injury or pathology")}

Als erstes Kriterium für die Klassifikation der zentralen Sensibilisierung ist eine unverhältnismäßig hohe Schmerzempfindung in Relation zum Ausmaß der eigentlichen Verletzung/Schädigung angeführt. Darunter zählt auch die Diskrepanz zwischen Grad der Alltagseinschränkung im Vergleich zu dem typischen pathophysiologischen Ausmaß einer vergleichbaren Schädigung (Nijs et al. 2014).

\section{Kriterium 2 ("Diffuse pain distribution + hyperalgesia / allodynia")}

In einem zweiten Schritt wird der Patient auf Hinweise für eine diffuse Schmerzausbreitung untersucht. Diese umfasst vornehmlich Schmerzen, die spiegelbildlich auf beiden Körperseiten vorkommen und neuroanatomisch nur schwer nachvollziehbar sind, sowie chronic widespread pain (Nijs et al. 2014). Dieser ist zum Beispiel durch Schmerz für mindestens drei Monate im Achsenskelett und in mindestens zwei kontralateralen Quadranten des Körpers definiert (Hunt et al. 1999) und wird häufig im Rahmen der Fibromyalgie-Diagnostik beispielsweise mithilfe von Fragebögen als Widespread Pain Index ermittelt (Häuser et al. 2012). Aber auch Schmerzzeichnungen sind ein probates Hilfsmittel, mit dem die Ausbreitung der Schmerzsymptomatik bei Patienten möglichst präzise dargestellt werden kann (Margolis et al. 1986). In unserer Studie wurden daher zur Diagnostik von generalisiertem Schmerzempfinden sowohl Anteile des Fibromyalgie-Fragebogens als auch digitale Schmerzkarten (Bryner 1994) genutzt. Die in den Kriterien genannten Schmerzphänomene wie die Allodynie und Hyperalgesie wurden in unserer Studie primär sowohl durch die Fragebögen als auch durch die Quantitativ-Sensorische Testung erfasst.

\section{Kriterium 3 ("Hypersensitivity of senses unrelated to the musculoskeletal system")}

Das dritte Kriterium prüft eine generelle Überempfindlichkeit auf Umweltreize wie Licht, Wärme, Kälte, elektrische Stimuli sowie Stress, Überladung und Emotion (Nijs et al. 2014). Dies kann einerseits durch eine ausführliche Anamnese, ergänzt durch den oben bereits angesprochenen Central Sensitization Inventory (Mayer et al. 2012) erfolgen. In dieser Arbeit wurden stattdessen Bausteine der Quantitativ-Sensorischen Testung genutzt, um Reize wie beispielsweise Kälte, Wärme und mechanischen Druck zu simulieren. In der Schmerzforschung etablierte Fragebögen, mit denen emotionale und psychologische Aspekte beleuchtet werden sollten, wurden entsprechend eingesetzt. 


\subsection{Zentrale Sensibilisierung und Arthrose}

Chronische Schmerzen bei muskuloskelettalen Erkrankungen wie der Arthrose waren in den vergangenen Jahrzehnten immer wieder Gegenstand der Forschung. Mittlerweile ist bekannt, dass zentrale Mechanismen Anteil am Übergang von akuten zu chronischen Schmerzen und deren Aufrechterhaltung haben können. Allerdings sind die pathophysiologischen Vorgänge weiterhin nur in Ansätzen verstanden und erfordern weitere Untersuchungen. Auch die systematische Beschreibung und einheitliche klinische Definition der zentralen Sensibilisierung befindet sich bisher erst in den Anfängen. Anwendbare Kriterien wie die oben beschriebenen sind wichtig für den klinischen Alltag, erfordern allerdings noch weiterführende Prüfung bevor diese regelhaft eingesetzt werden können. Im Verhältnis zu Schmerzen bei Kniearthrose wurde die Hüftarthrose zudem wissenschaftlich lange vernachlässigt. Bis auf wenige ältere Studien die sich zumindest in Teilen mit chronic widespread pain bei Koxarthrose beschäftigten (O’Driscoll und Jayson 1974, Kosek und Ordeberg 2000a) wurde lange Zeit wenig Neues publiziert. Aktuell wird wieder mehr auf dem Gebiet geforscht, allerdings vornehmlich mit Probanden die unmittelbar vor einem Gelenksersatz stehen (Wylde et al. 2017) oder diesen bereits erhalten haben (Erlenwein et al. 2017), häufig mit einem Fokus auf die postoperative Situation. Kaum etwas ist bekannt über Anzeichen zentraler Komponenten in der Schmerzgenese bei Menschen im oft jahrelangen Vorlauf einer operativen Therapie und im Rahmen der Stellung einer OPIndikation selbst. Kenntnisse zur Rolle der zentralen Sensibilisierung könnten zu Strategien zur Verzögerung und Optimierung der OP-Indikation führen.

Zur Operationalisierung von Zeichen der zentralen Sensibilisierung bei Menschen mit Hüftarthrose wurden in dieser Studie etablierte Verfahren aus der Methodik der QuantitativSensorischen Testung verwendet, die in der Lage sind prinzipiell alle Submodalitäten der Somatosensorik zu erfassen (Rolke et al. 2006a). So können die Ergebnisse der Testung Hinweise auf Defizite in verschiedenen Bereichen der Leitungsbahnen der Somatosensorik, wie z.B. in „Afferenzen (...) des Hinterstrangsystems, (...) oder des Vorderseitenstrangsystems, (in der) periphere(n) Sensibilisierung, (...) (oder) der endogenen Schmerzhemmung“" geben (Treede 2013a, S. 88). 


\subsection{Ziel der Dissertation}

Ziel dieser Dissertation war es daher, (1) mithilfe von hüftgelenksnah und distal gemessenen Temperatur- und Druckschmerzschwellen das Vorliegen einer generalisiert erhöhten Schmerzwahrnehmung bei Menschen mit Hüftgelenksarthrose im Gruppenvergleich mit schmerzfreien Menschen als Hinweis auf eine zentrale Sensibilisierung zu prüfen. Die ursprünglich zusätzlich geplante Messung des nozizeptiven Flexorenreflexes konnte aus technischen Gründen nicht valide durchgeführt werden. Als weiteres Anzeichen einer zentralen Sensibilisierung wurde (2) die Schmerzausdehnung standardisiert erfasst. Da im Rahmen einer zentralen Sensibilisierung auch die Verarbeitung von Stress, Emotionen und anderen Sinneseindrücken verändert sein kann, wurden mithilfe von Fragebögen (3) Hinweise auf schmerzpsychologische Komorbiditäten und Chronifizierungsfaktoren, sowie das Vorliegen einer neuropathischen Schmerzcharakteristik gesucht.

Erwartet wurde dabei ein Nachweis einer generalisierten und damit zentralen Sensibilisierung innerhalb des nozizeptiven Systems, messbar als erniedrigte Druck- und Hitzeschwellen und erhöhte Schmerzschwellen für Kältereize, wie zuvor auch schon für andere chronische Schmerzerkrankungen beschrieben. Ebenso konnte ein korrelativer Zusammenhang mit der Schmerzausdehnung und anderen Zeichen einer zentralen Sensibilisierung bzw. Hinweise auf eine beginnende Chronifizierung erwartet werden. Die Ergebnisse sollten folglich im Kontext von anderen chronischen Schmerzerkrankungen bewertet und sofern möglich, klinische Konsequenzen des Vorliegens einer zentralen Sensibilisierung bei Menschen mit Koxarthrose abgeleitet werden. 


\section{Material und Methoden}

\subsection{Studie}

\subsubsection{Hypothese}

Studienhypothese war, dass Menschen mit Hüftarthrose im direkten Vergleich mit einer Kontrollgruppe in den Untersuchungen der Quantitativ-Sensorischen Testung Hinweise auf eine Übererregbarkeit innerhalb des schmerzverarbeitenden Systems und damit Zeichen einer zentralen Sensibilisierung zeigen. Die Schmerzempfindlichkeit wurde lokoregional im Bereich der betroffenen Hüfte sowie gelenkfern ipsi- und kontralateral gemessen. Die Schmerzausdehnung wurde mittels Fragebogen und Schmerzzeichnung quantifiziert, schmerzpsychologische Faktoren mittels Fragebogen. Es wurde postuliert, dass Patienten mit Koxarthrose eine zentrale Sensibilisierung aufweisen, die sich in einer generalisierten Schmerzausdehnung und einer erhöhten Schmerzempfindlichkeit zeigt. Der Einfluss von Geschlecht und psychologischen Faktoren sollte dabei berücksichtigt werden.

\subsubsection{Studiendesign und -ethik}

Bei der durchgeführten Studie handelte es sich um eine explorative Querschnittsstudie mit insgesamt 30 Patienten mit einer nach ICD-10 diagnostizierten Koxarthrose und 18 gesunden Probanden im Vergleich.

Die Datenerhebung fand von Oktober 2015 bis März 2016 in den Räumlichkeiten der SchmerzTagesklinik und -Ambulanz, Klinik für Anästhesiologie, der Universitätsmedizin Göttingen statt. Die Studie wurde entsprechend der Declaration of Helsinki (WMA 2013) durchgeführt und von der Ethik-Kommission der Universitätsmedizin Göttingen durch Antragsnummer 27/8/15 im Vorfeld geprüft und positiv bewertet. Einschlussfähige Probanden wurden vor Beginn der Studie über mögliche Risiken aufgeklärt und gaben ihr schriftliches Einverständnis.

\subsubsection{Rekrutierung der Probanden}

Die Rekrutierung der Probanden erfolgte über die Schmerz-Tagesklinik und -Ambulanz, Klinik für Anästhesiologie der Universitätsmedizin Göttingen, ambulante orthopädische und physiotherapeutische Fachpraxen, ambulante Rehabilitationseinrichtungen und Zeitungsannoncen in regionalen Tageszeitungen. Probanden für die Kontrollgruppe wurden über Aushänge in der Universitätsmedizin Göttingen und Zeitungsannoncen geworben. 


\subsubsection{Einschluss- und Ausschlusskriterien}

Teilnahmevoraussetzung war die fachorthopädische ICD-Diagnose einer einseitig betonten Koxarthrose. Auf die Beurteilung einer neuen Bildgebung bei Studieneinschluss zur Korrelation mit den Studienparametern wurde bewusst verzichtet. Ausschlusskriterium war eine andere schmerzassoziierte Diagnose wie beispielsweise eine rheumatoide Arthritis, chronische Nackenoder Rückenschmerzen oder ein Fibromyalgie-Syndrom. Auch schwerwiegende kardiovaskuläre, respiratorische, neurologische oder psychiatrische und kognitive Vorerkrankungen bzw. Einschränkungen waren Grund zum Ausschluss.

Probanden mit einem BMI $>32 \mathrm{~kg} / \mathrm{m} 2$ oder einem Alter unter 40 bzw. über 70 Jahren wurden ebenfalls ausgeschlossen. Für die Kontrollgruppe wurden in Alter und Geschlecht passende Probanden rekrutiert. Vor Einladung zur Studienteilnahme wurden die Probanden telefonisch auf das Vorliegen von Ein- und Ausschlusskriterien geprüft.

\subsubsection{Typischer Ablauf einer Einzelsitzung}

Die durchschnittliche Dauer einer Sitzung betrug etwa zwei Stunden. Nach Begrüßung des Probanden erfolgte zunächst die erforderliche Aufklärung über Datenschutz und die bevorstehenden Untersuchungen inklusive möglicher Risiken. Nach Gabe des schriftlichen Einverständnisses wurde allen Probanden mit Hüftarthrose zunächst der Fragenbogenkatalog ausgehändigt, für den die Teilnehmer ausreichend Zeit zur Bearbeitung erhielten. Fragen konnten im Anschluss beantwortet werden. Als nächstes wurden die Patienten mit Koxarthrose gebeten, eine digitale Schmerzzeichnung der Schmerzausbreitung in den letzten vier Wochen zu erstellen. Nach Entkleidung begann die eigentliche Untersuchung. Dafür wurden die Teilnehmer gebeten, sich auf eine für alle Probanden gleichen Untersuchungsliege mit circa $30^{\circ}$ erhöhtem Kopfteil zu legen. Nach circa fünf minütiger Ruhepause wurde am rechten Arm der Blutdruck nach Riva Rocci gemessen. Im Anschluss folgten nach den jeweiligen Instruktionen die Quantitativ-Sensorische Testung (QST), zunächst mit den Thermotestungen, dann mit den Messungen der Druckschmerzschwellen und zuletzt die Messung des norizeptiven Flexorreflexes. 


\subsection{Schmerzausdehnung}

Durch den Probanden mit Hüftarthrose wurde eine möglichst realistische Schmerzzeichnung erstellt, für die eine standardisierte Abbildung eines frontal, dorsal und lateral abgebildeten Menschen in Neutral-Null-Position vorgegeben war.

Verwendet wurde hierfür die Software SketchBook. Pro auf einem Ipad Air 2, Model 1566, Late 2014 mit einem CS100B Stift für Tablets. Für diese Methode konnte eine gute Test-Retest-Reliabilität für Schmerzausmaß und Schmerzlokalisation erfasst werden (Barbero et al. 2015).

Der Proband wurde unter standardisierter Versuchserklärung gebeten, in den entsprechenden Abbildungen mit der vorgegebenen Markierhilfe all diejenigen Orte in blau einzufärben, an denen in den letzten vier Wochen bei Alltagstätigkeiten Schmerzen auftraten. Es wurde darauf hingewiesen die Schmerzkarte sorgfältig und vollständig auszufüllen.

Im Anschluss sollte der Punkt des maximalen Schmerzes mit der Markierhilfe in grün markiert werden.

Die Probanden hatten im Vorfeld ausreichend Zeit sich mit der Handhabung vertraut zu machen und konnten im Anschluss solange Änderungen vornehmen, bis die erstellte Karte der eigenen Schmerzsituation entsprach. Die Auswertung erfolgte über die Freeware P_Bochan, die anhand der gefärbten Pixel das Ausmaß der Schmerzlokalisation erkennt und eine SchmerzFrequenz-Karte erstellt. Eine Farbcodierung ermöglicht die Angabe, wie viel Prozent der Teilnehmer in einer bestimmten Körperregion Schmerzen angaben. Beispiele der Vorlagen sind in Abbildung 1 und 2 gezeigt.

Weiterhin berechnet das Programm einen Gesamtwert der Schmerzausdehnung im Verhältnis zu schmerzfreien Regionen in Prozent. In dieser Arbeit fand für die weiteren Berechnungen nur dieser Gesamtwert für jeden Teilnehmer Verwendung. 


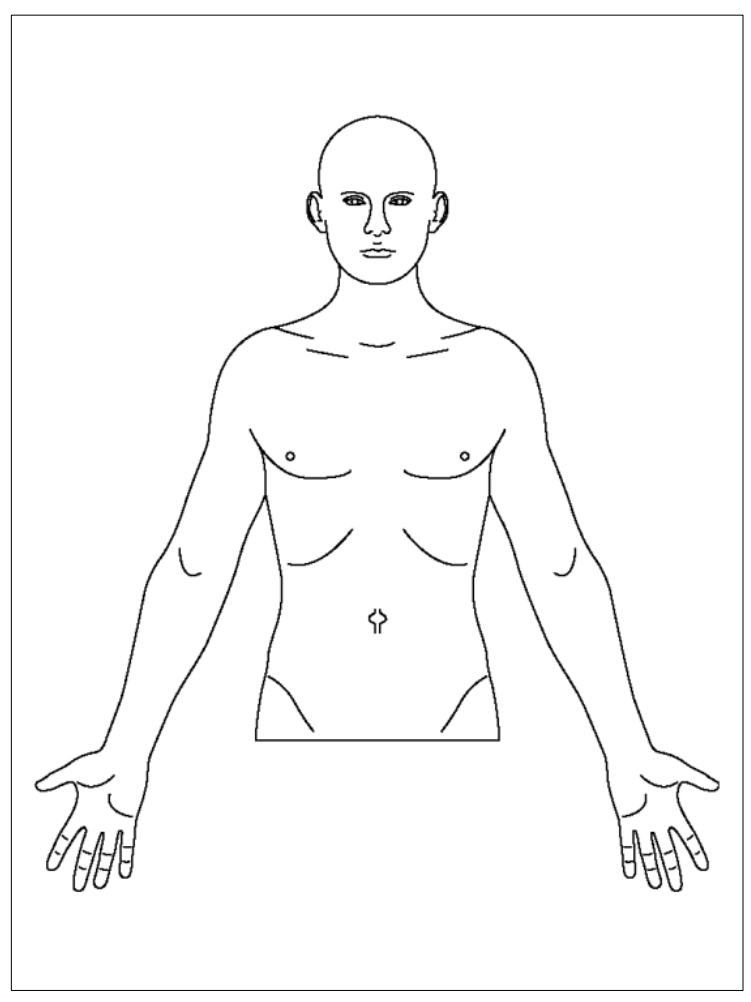

Abbildung 1: Beispiel Vorlage Schmerzzeichnung

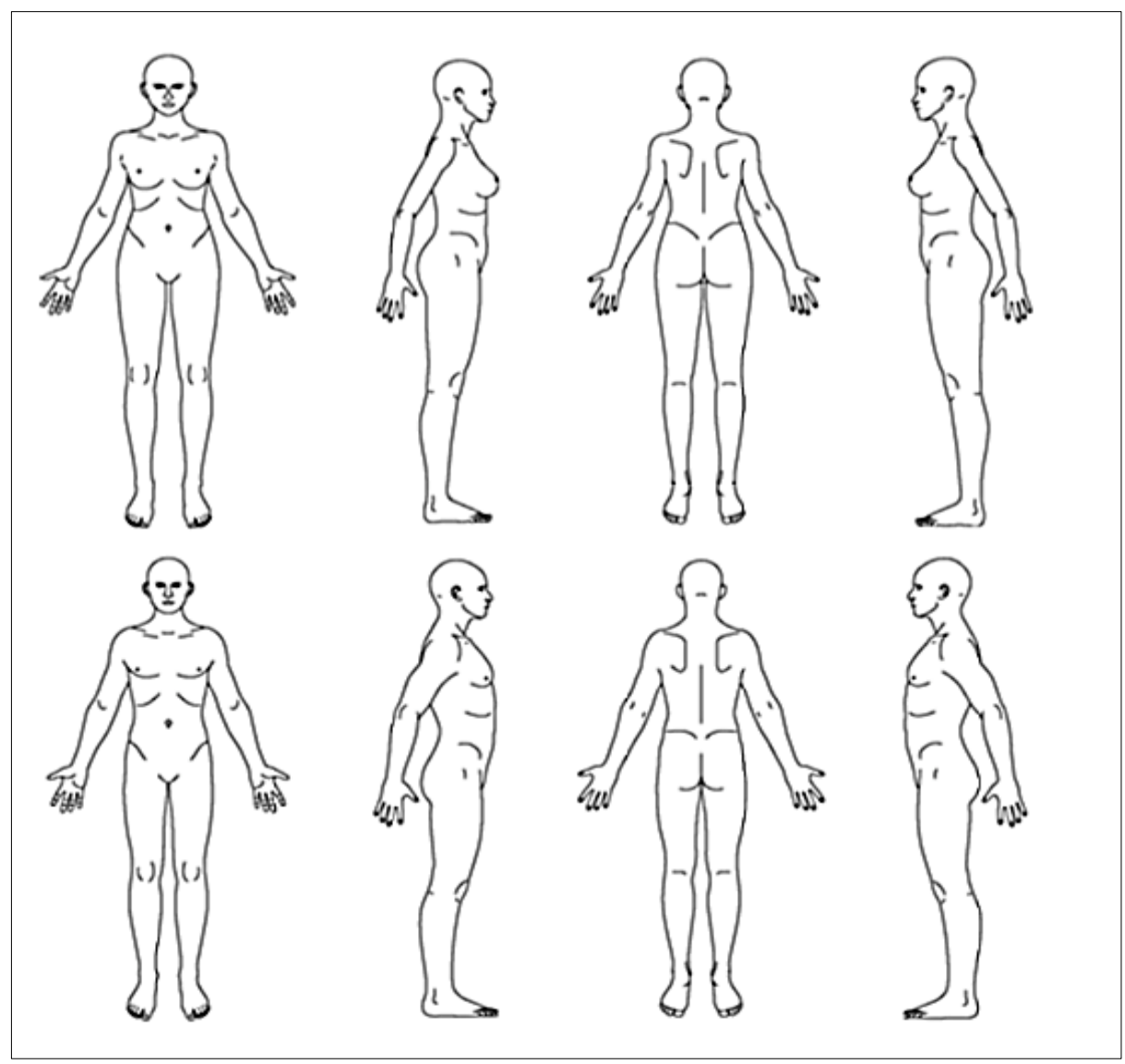

Abbildung 2: Beispiele der vorgefertigten Schablonen für beide Geschlechter 


\subsection{Fragebögen}

Wie oben bereits angedeutet, sollten die in der Studie verwendeten Fragebögen Hinweise auf mögliche Begleitmorbiditäten und Chronifizierungsfaktoren aus dem schmerzpsychologischen oder emotionalen Kontext geben. Alle Fragebögen bis auf die verwendeten Anteile des Deutschen Schmerzfragebogens sind im Anhang beigefügt.

Die Probanden der Gruppe mit Koxarthrose wurden gebeten folgende Fragebögen in standardisierter Reihenfolge zu bearbeiten:

1. Anteile des Deutschen Schmerafragebogens (DSF): Hier wurden zunächst allgemeine Fragen zu Alter, Geschlecht, Zeitpunkt der Diagnose der Arthrose, sowie weitere Fragen zu Schmerzintensität und -häufigkeit gestellt (Pfingsten et al. 2006).

2. Oxford Hip Score (OHS): In der deutschen Version des Oxford Hip-Scores (OHS-D) nach der Übersetzung von Naal et al. 2009 werden insgesamt zwölf Fragen (mit bis zu 5 Punkten pro Frage, maximal 60 Punkte) zu Schmerzen in der Hüfte im Alltag und eventuellen Einschränkungen bezüglich täglicher Aktivitäten wie Ankleiden, Essen oder Laufen gestellt (Naal et al. 2009). Je höher der Wert, desto höher der Grad der Alltagseinschränkung der Patienten mit Hüftschmerzen.

3. Pain Catastrophizing Scale (PCS): Die Pain Catastrophizing Scale wurde in deutscher Version von Meyer et al. 2008 veröffentlicht und hinsichtlich ihrer Objektivität, Validität und Reliabilität evaluiert. In diesem Fragebogen werden dem Befragten insgesamt dreizehn Fragen gestellt, die auf affektive Komponenten, Gedanken und Gefühle bezüglich des Schmerzerlebens abzielen. Insgesamt existieren in der Auswertung die drei Subskalen helplessness, rumination und magnification die alle mit bis zu 4 Punkten pro Frage bewertet werden. Eine höhere Punktzahl bedeutet einen höheren Grad des Katastrophisierens. Es werden drei Punktwerte für die Subskalen und eine Gesamtpunktzahl mit maximal 52 Punkten ermittelt (Meyer et al. 2008). Eine Gesamtpunktzahl $>28$ gilt als auffällig.

4. Fibromyalgie-Syndrom Survey-Questionnaire: Dieser Fragebogen erfasst die vorläufigen Kriterien des American College of Rheumatology (ACR) zur Diagnose eines Fibromyalgie-Syndroms (FMS) und umfasst zwei Subskalen.

Mit dem (1) Widespread-Pain Index (WPI) werden neunzehn schmerzhafte oder berührungsempfindliche Orte des Körpers durch den Patienten ausgewählt und getrennt nach rechter und linker Körperseite bewertet und aufsummiert. 
Weiterhin werden mithilfe der Subskala (2) Symptom Severity Score (SSS) Kopfschmerzen, depressive Verstimmungen und viszerale Schmerzen des letzten halben Jahres sowie Tagesmüdigkeit, nicht erholsamer Schlaf und Gedächtnisprobleme bezogen auf die letzte Woche evaluiert. Für den Fragebogen in der deutschen Übersetzung konnten gute psychometrische Eigenschaften auch für andere muskuloskelettale Erkrankungen nachgewiesen werden (Häuser et al. 2012). Patienten, die die Kriterien dieses Fragebogens für das Vorliegen eines FMS erfüllen sollten einen Widespread Pain Index (WPI) mit $\geq 7 / 19$ Schmerzorten und einen Symptom Severity Score (SSS) $\geq 5 / 12$ bzw. einen WPI zwischen $\geq 3-6 / 19$ und SSS $\geq 9 / 12$ aufzeigen (Häuser et al. 2012).

5. painDETECT: Dieser Fragebogen ist vornehmlich im Kontext von neuropathischen Schmerzen validiert (Freynhagen et al. 2016) und wurde daher in unserer Studie vor allem für die Schmerzcharakterisierung und den Ausschluss wahrscheinlich neuropathischer Schmerzen verwendet. Allerdings ist er auch bei Patienten mit FMS häufig positiv, was als Hinweis auf eine zentrale Sensibilisierung gewertet wird (Amris et al. 2010).

6. Tampa Scale for Kinesiophobia (TSK): Die deutsche Version der Tampa Scale for Kinesiophobia, die als geeignetes Instrument für die Bestimmung von Ängsten vor erneuten Verletzungen bei Aktivität gilt (Rusu et al. 2014), soll Verhaltensweisen erkennen, in denen Patienten mit Schmerz typischerweise Angst vermeiden.

7. Chronic Pain Acceptance Questionnaire $(C P A Q) /$ Schmerzakzeptanz Fragebogen: Der CPAQ misst die Schmerzakzeptanz bei Menschen mit chronischem Schmerz und wurde in der deutschen Fassung hinsichtlich seiner psychometrischen Eigenschaften für gut bewertet (Nilges et al. 2007).

8. SF-36 Health Survey (Lebensqualität): Der SF-36 wurde als sinnvolles generisches Instrument zum Nachweis von gesundheitsspezifischer Lebensqualität validiert (Bullinger et al. 1995) und liefert mit den Summationsskalen Mental Component Scale (MCS) und Physical Component Scale (PCS) sowohl Hinweise auf körperliche als auch psychische Beeinträchtigungen.

9. Depression, Anxiety, Stress Scales (DASS): Nach Nilges und Essau 2015 stellt der DASS eine ,zuverlässige und ökonomisch(e), sowohl hinsichtlich Zeitaufwand als auch Kosten(,) (...) Alternative zu bisherigen Verfahren“ (Nilges und Essau 2015, S. 1) dar, 
um erste Anzeichen einer Depression oder dysthymer Verstimmung bei Patienten mit chronischem Schmerz zu detektieren. Die Subskalen Depression (cut-off $>10$ Punkte), Angst (cut-off $>6$ Punkte) und Stress (cut-off $>10$ Punkte) können hierbei unterschieden werden.

10. Von Korff Scale (VKS): Die VKS wird als ein Instrument gesehen mit dem chronischer Schmerz in verschiedene Schweregrade (I-IV), abgestuft getrennt nach Beeinträchtigung durch Schmerz und Schmerzintensität, eingeteilt werden kann (Klasen et al. 2004).

Grad I entspricht dabei einer niedrigen Schmerzintensität bei geringer körperlicher Behinderung, Grad II einer hohen Schmerzintensität bei noch geringer körperlicher Behinderung, III einer hohen körperlichen Behinderung mit moderater Beeinträchtigung und Grad IV einer starken körperlichen Beeinträchtigung die zu sehr starken Einschränkungen der Alltagsfunktionen führt.

Während Grad I und II klinisch als funktionaler chronischer Schmerz interpretiert werden, wird ab Grad III von einem dysfunktionalen chronischen Schmerz gesprochen (Von Korff et al. 1992). 


\subsection{Quantitativ-Sensorische Testung}

Die Batterie der Quantitativ-Sensorischen Testung, entwickelt durch den Deutschen Forschungsverband neuropathischer Schmerz (DFNS), stellt eine standardisierte Möglichkeit dar, Wahrnehmungsschwellen, Reiz- und Antwortkurven, sowie Schmerzschwellen zu erfassen. Sowohl Sensibilitätsverluste, aber auch typische Phänomene wie Allodynie, Hyperalgesie und Hyperpathie können hiermit detektiert werden (Rolke et al. 2006a). Es ist möglich, gegebenenfalls vorhandene Hinweise auf zentrale Schmerzursachen wie z.B. mechanische und thermische Hyperalgesie oder andere Positivzeichen $\mathrm{zu}$ messen. Als klinisches Standardinstrument findet es daher Verwendung im Rahmen der Diagnostik neuropathischer Schmerzen (Pfau et al. 2012) und anderer chronischer Schmerzerkrankungen (Pfau et al. 2009). Ein weiterer Vorteil der nicht-invasiven Testserie ist, dass diese durch hohe Standardisierung reproduzierbare Ergebnisse relativ unabhängig vom Untersucher liefert und die gesamte Bandbreite der nozizeptiven Achse analysieren kann (Verdugo und Ochoa 1992, Ziegler et al. 1999, Rolke et al. 2006b). Es konnte ferner gezeigt werden, dass die Quantitativ-Sensorische Testung einen hohen Stellenwert für die Forschung im Kontext von Schmerzen bei muskuloskelettalen Erkrankungen wie der Arthrose hat (Pavlaković und Petzke 2010). Zudem wurden längst Normalbereiche definiert, die eine Interpretation gewonnener Daten erleichtern können (Magerl et al. 2010).

In unserer Studie wurden nur eine Auswahl von Tests nach dem modifizierten Protokoll nach Rolke et al. 2006 verwendet. In einer standardisierten Reihenfolge (Rolke et al. 2006a) erfolgte die multilokuläre Bestimmung von Schwellenwerten für Kälte- und Hitzeschmerz, sowie Druckschmerz (Abbildung 3).

Aufgrund der Messung an mehreren Orten verzichteten wir aus Praktikabilitätsgründen auf die Bestimmung der übrigen Parameter. 


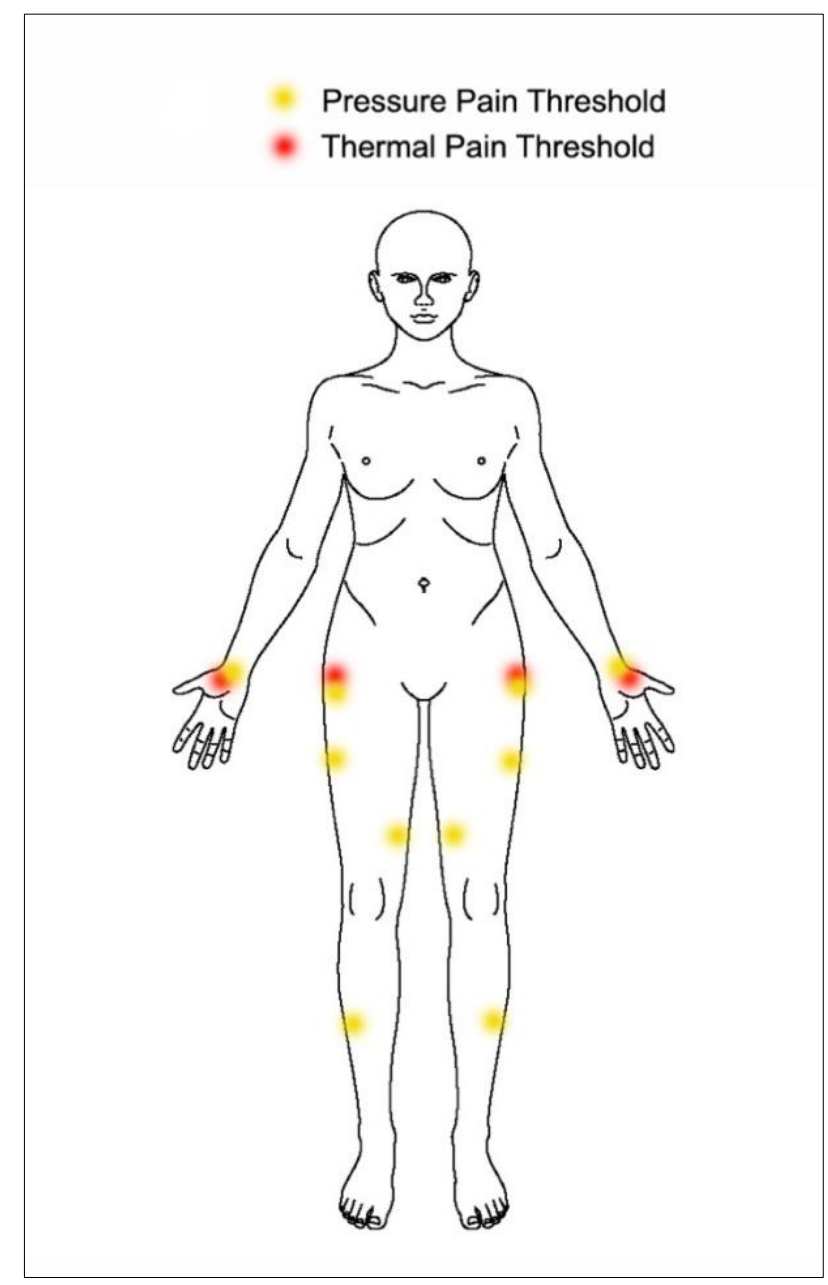

Abbildung 3: Übersicht über die verschiedenen Untersuchungspunkte von ventral (Der M. gluteus medius feblt in dieser Ansicht.)

\subsubsection{Thermale Schwellenwerte}

Dem QST-Protokoll folgend, wurden zunächst mithilfe des Gerätes Medoc TSA-II (Ramat Yishai, Israel, Abbildung 4) die thermischen Schwellenwerte beidseits bestimmt.

Dafür wurde die Thermode (Abbildung 5) mit einer etwa $3 \mathrm{~cm}$ x $3 \mathrm{~cm}$ großen Metallfläche auf der betreffenden Körperstelle fixiert. Das Gerät kann mithilfe des Peltier-Effektes (Fruhstorfer et al. 1976) die der Haut zugewandten Fläche einerseits erhitzen, aber auch abkühlen. Vor Beginn der eigentlichen Erhebung wurde jeweils eine Probemessung an einem neutralen Hautareal zur Gewöhnung an die Prozedur durchgeführt.

Mithilfe eines Schalters konnte der Patient die Messung jederzeit stoppen. Eine obere Temperaturgrenze wurde bei $50{ }^{\circ} \mathrm{C}$ gesetzt, während die untere Grenze bei $0{ }^{\circ} \mathrm{C}$ lag. Die Ausgangstemperatur lag vor jedem Durchgang bei $32{ }^{\circ} \mathrm{C}$. Die Temperatur veränderte sich mit $1{ }^{\circ} \mathrm{C} / \mathrm{s}$. Die Messungen an der Hand wurden bilateral am Thenar des Daumens durchgeführt, an der Hüfte wurde beidseits im Bereich des Trochanter major gemessen (Abbildung 3). 
Folgende Untersuchungen wurden unter Verwendung der standardisierten Instruktionen durchgeführt (Rolke et al. 2006a):

\subsubsection{Cold Detection Threshold (CDT) und Warm Detection Threshold (WDT)}

Zunächst wurde die Thermode pro Sekunde um etwa $1{ }^{\circ} \mathrm{C}$ abgekühlt. Bei dieser ersten Messung sollte der Patient sofort auf die Stopptaste drücken, sobald er erstmals eine Abkühlung auf der Haut spüren konnte. Im Anschluss wurde nach kurzer Pause in gleicher Weise die Schwelle bestimmt, ab der der Patient erstmals eine Erwärmung spüren konnte.

\subsubsection{Cold Pain Threshold (CDT) und Heat Pain Threshold (HPT)}

In der nächsten Testung sollte der Proband sofort auf die Stopptaste drücken, sobald sich während der langsamen Abkühlung der Thermode $\left(1^{\circ} \mathrm{C} / \mathrm{s}\right)$ zu der Empfindung von Kälte, eine schmerzhafte Empfindung in Form eines Brennens, Stechens, Bohrens oder Ziehens einstellte. Bei der anschließenden Testung der Hitzeschmerzschwelle sollte der Proband die Temperaturrampe stoppen, wenn zu der Hitze- oder Wärmeempfindung eine schmerzhafte Empfindung in Form eines Brennens, Stechens, Bohrens oder Ziehens hinzukam. 


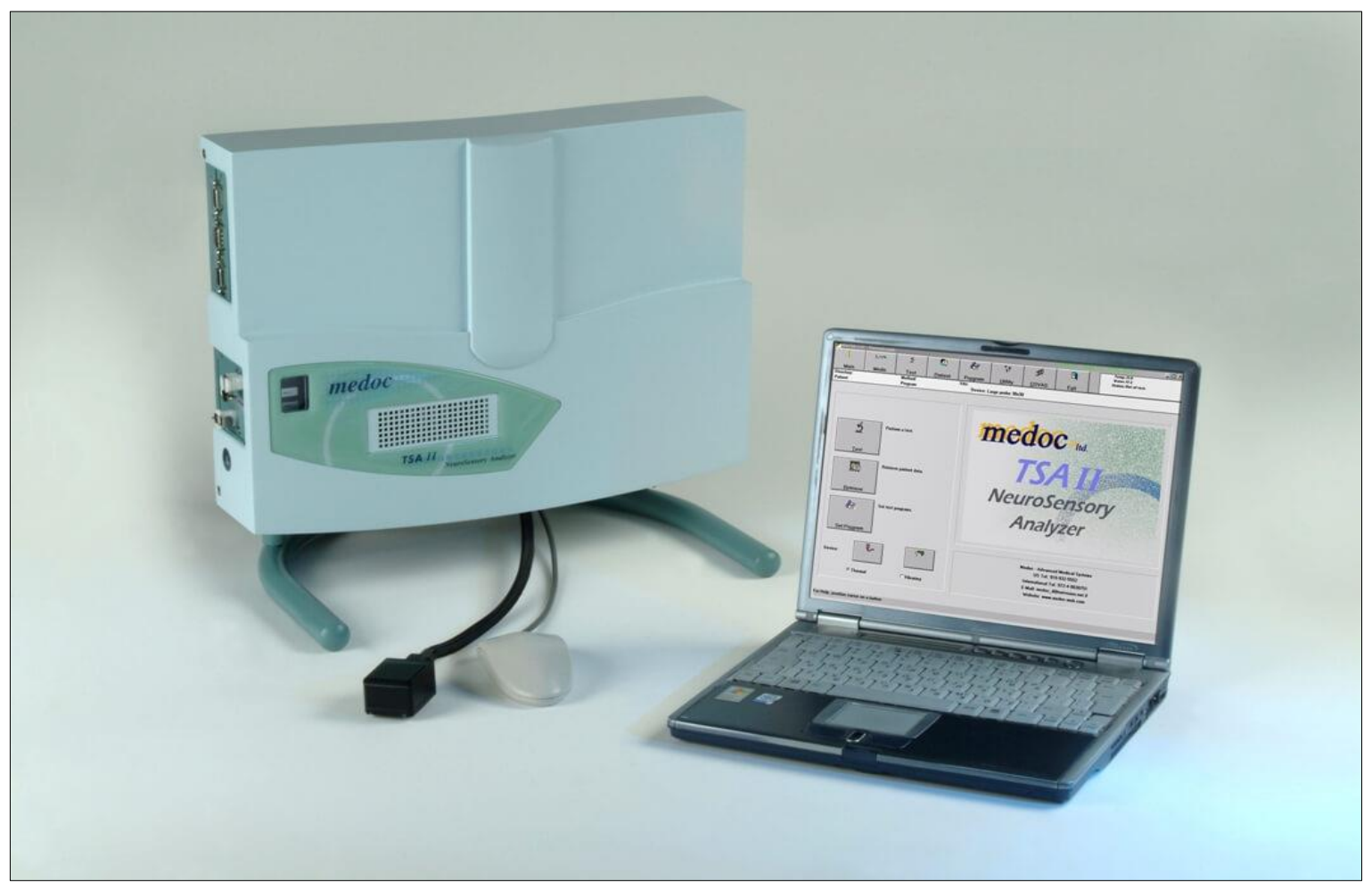

Abbildung 4: Der Thermal Sensory Analyzer TSA-II, Firma Medoc

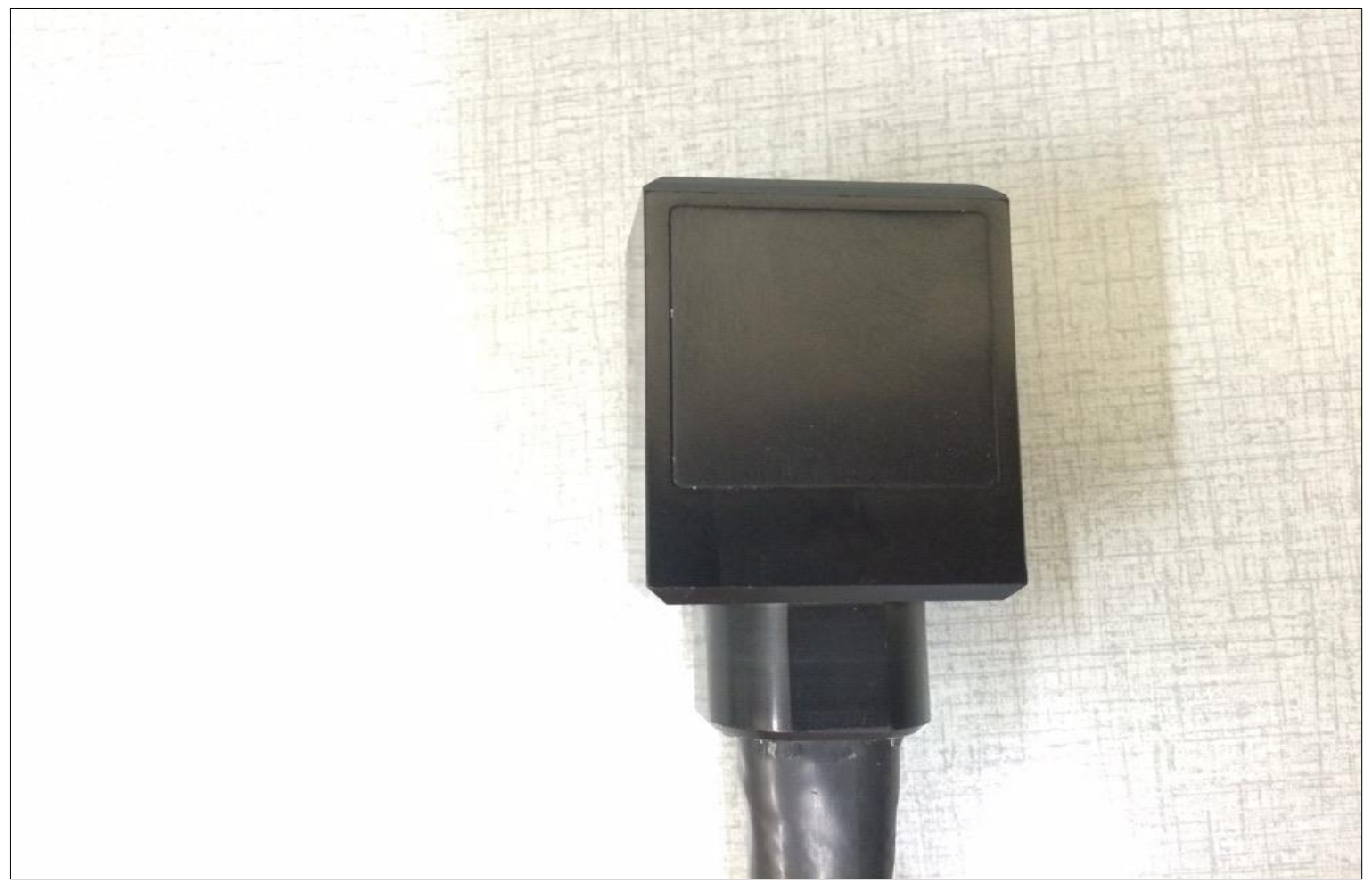

Abbildung 5: Die Peltier-Thermode 


\subsubsection{Druckschmerzschwellen/ Pressure Pain Threshold (PPT)}

Die Druckschmerzschwelle ist definiert als der lokal applizierte Druck, welcher Schmerz oder Unbehagen erzeugt (Fischer 1987). Nachgewiesen wurde, dass die manuelle Messung von Druckschmerz mithilfe dieser einfachen und verhältnismäßig preiswerten Methode Hinweise auf muskuloskelettalen Tiefenschmerz mit reproduzierbaren Ergebnissen liefern kann (Rolke et al. 2005). Sie findet heutzutage als klinisches Standardinstrument in der Schmerzforschung und -diagnostik Anwendung. Die Druckschmerzschwelle wird methodisch von der Größe der Kontaktplatte und der Druckanstiegsgeschwindigkeit beeinflusst.

In dieser Studie wurde mit einer $1 \mathrm{~cm} 2$ große Kontaktfläche eines stumpfen mechanischen Stimulators (Algometer by Somedic, Schweden, Abbildung 6) die in $90^{\circ}$ zur Hautoberfläche applizierte Kraft pro Sekunde kontrolliert um etwa $50 \mathrm{kPa} / \mathrm{s}$ gesteigert bis der Patient erstmalig angab, dass zu der Qualität von Druck zusätzlich die Qualität eines Brennens, Bohrens, Stechens oder Ziehens hinzugekommen ist. In diesem Fall wurde die Messung gestoppt und der erreichte Schwellenwert über ein integriertes Display direkt abgelesen. Zur Kontrolle der Druckanstiegsgeschwindigkeit bietet das Gerät eine optische Darstellung. Pro Körperregion wurde die Testung drei Mal durchgeführt und ein Mittelwert generiert. Über folgenden Messorten wurde jeweils im Seitenvergleich die Druckschmerzschwelle bestimmt (Abbildung 3).

- Medialer Anteil des Daumenballens (Thenar)

- Trochanter major

- M. gluteus medius (über dem proximalen Muskelbauch, etwa $3 \mathrm{~cm}$ distal des Beckenkammes, zwischen Spina iliaca anterior superior und Trochanter major)

- M. vastus medialis (etwa $3 \mathrm{~cm}$ oberhalb der Patella, mediale Seite des Oberschenkels)

- M. vastus lateralis (in etwa der Mitte zwischen dem Trochanter major und dem Epicondylus lateralis femoris)

- M. tibialis anterior (in etwa der Mitte zwischen seiner oberen Befestigung an der Tibia und seiner Sehne im oberen Drittel des Muskelbauches). 
Die Reihenfolge der Testung wurde insofern randomisiert, indem die Bestimmung der Druckschmerzschwellen von Proband zu Proband im Wechsel von proximal nach distal oder umgekehrt erfolgte. Aufgrund der besseren Übersichtlichkeit wurden innerhalb des Ergebnisteils hüftgelenksnahe Druckschmerzschwellen, also der PPT oberhalb des Trochanter major und des M. gluteus medius, zu einem Messpunkt zusammengefasst (Mittelwert beider Messungen). Auch für periphere, nicht direkt mit dem Hüftgelenk assoziierte Druckschmerzschwellen wurden zwei Druckschmerzschwellen (PPT über dem Thenar und PPT über dem M. tibialis anterior der nicht betroffenen Seite) zu einem Punkt als Mittelwert der Messpunkte zusammengefasst.

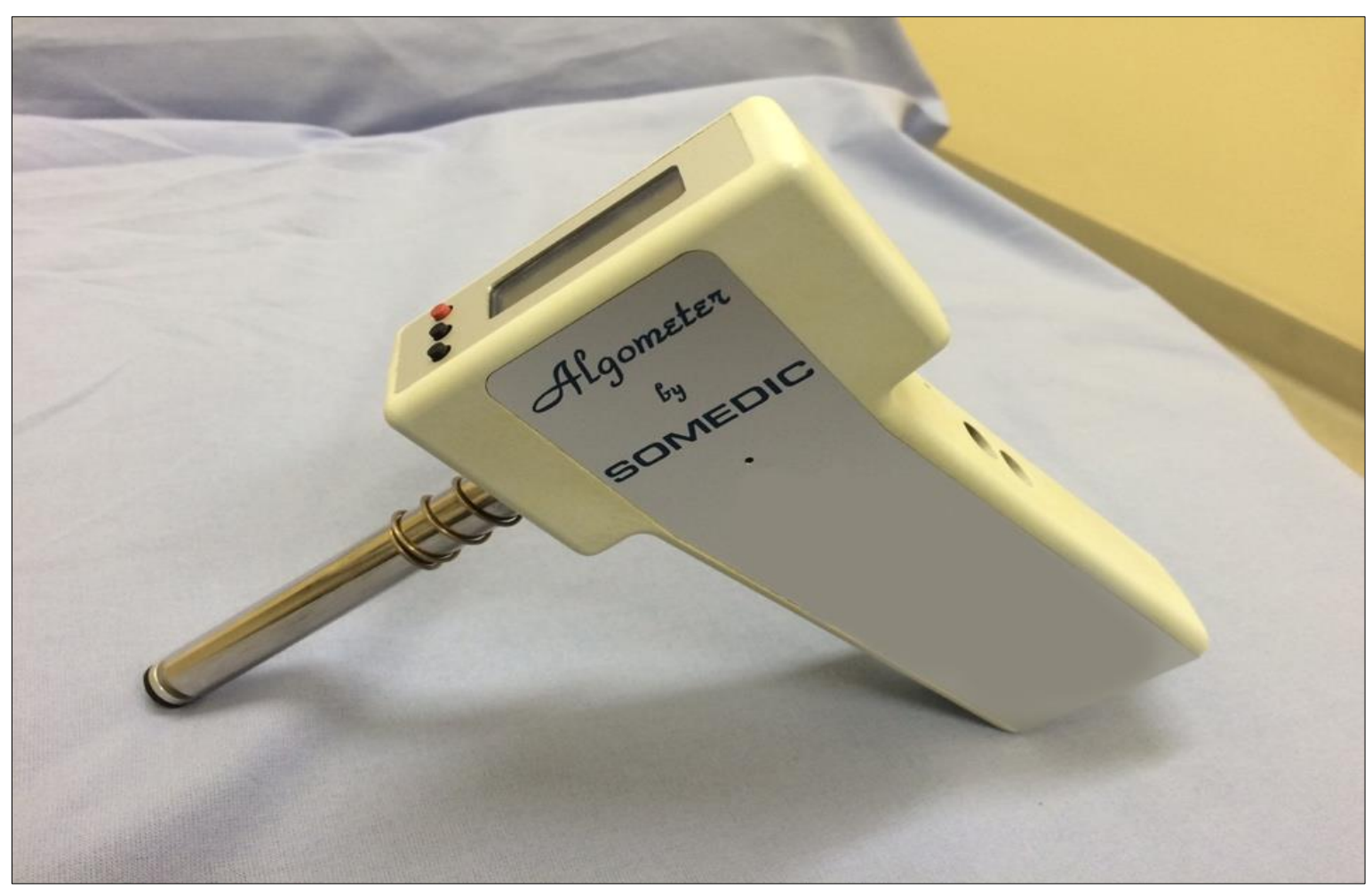

Abbildung 6: Das Algometer der Firma Somedic, Sweden 


\subsection{Nozizeptiver Flexorenreflex}

Studien konnten zeigen, dass die Messung des nozizeptiven Flexorenreflexes (RIII) bei Menschen in sedierten oder narkotisierten Zustand Hinweise auf die Narkosetiefe bzw. auf die Antinozizeption unter Narkose geben kann (von Dincklage et al. 2009a). Weiterhin konnte gezeigt werden, dass die Messung auch Erkenntnisse über das Verhältnis objektiver und subjektiver Schmerzschwellen im Rahmen chronischer Schmerzerkrankungen liefern kann (Sandrini et al. 2005, Biurrun Manresa et al. 2011). Im Kontext chronisch muskuloskelettaler Erkrankungen, wie beispielsweise der Hüftgelenksarthrose, fehlt es dem Verfahren bislang aber noch an Evaluation, sodass wir die Testung des Reflexes in dieser Studie durchführten.

Es wurde das Gerät Paintracker der Firma Dolosys, Berlin verwendet, das nach dem in der Publikation von von Dincklage et al. aus dem Jahr 2009 (von Dincklage et al. 2009b) beschriebenen Algorithmus Schwellen des nozizeptiven Flexorenreflexes ermitteln kann. Hierfür war nach ausreichender Vorbereitung der Haut durch Rasur und Anwendung einer speziellen Paste zur Entfernung von Hautunreinheiten die Platzierung von Reizelektroden im standardisierten Bereich des Außenknöchels und entsprechender Messelektroden oberhalb des M. bizeps femoris am ipsilateralen Bein erforderlich (Abbildung 7).

Nach transkutaner elektrischer Stimulation des Nervus suralis im Bereich des Außenknöchels wurde eine elektromyographische Reizantwort des M. bizeps femoris gemessen und auf dem integrierten Display (Abbildung 8) graphisch dargestellt. Durch entsprechende automatisierte Wiederholung der Untersuchung mit unterschiedlichen Reizstärken wurde dadurch die theoretische Schmerzschwelle in $\mathrm{mA}$ ermittelt. Dieses Verfahren wurde für beide Beine separat durchgeführt.

Leider ergaben sich im Verlauf der Testung in dieser Studie Probleme mit der Ableitungsqualität der Elektroden bei Probanden, bei denen trotz intensiver Präparation der Haut massive Artefakte die Messung beeinträchtigten. Sowohl Gesunde als auch Probanden mit Arthrose beschrieben darüber hinaus die Untersuchung als äußerst unangenehm, sodass wir nicht von allen Teilnehmern Daten generieren konnten. Auch bei der Auswertung der Daten ergaben sich Probleme, die am ehesten mit dem zum Teil noch fehlerhaften Algorithmus in Verbindung zu bringen sind.

Wir verließen daher die beschriebene Methode und verzichten in dieser Arbeit vollständig auf die Darstellung der Ergebnisse. 


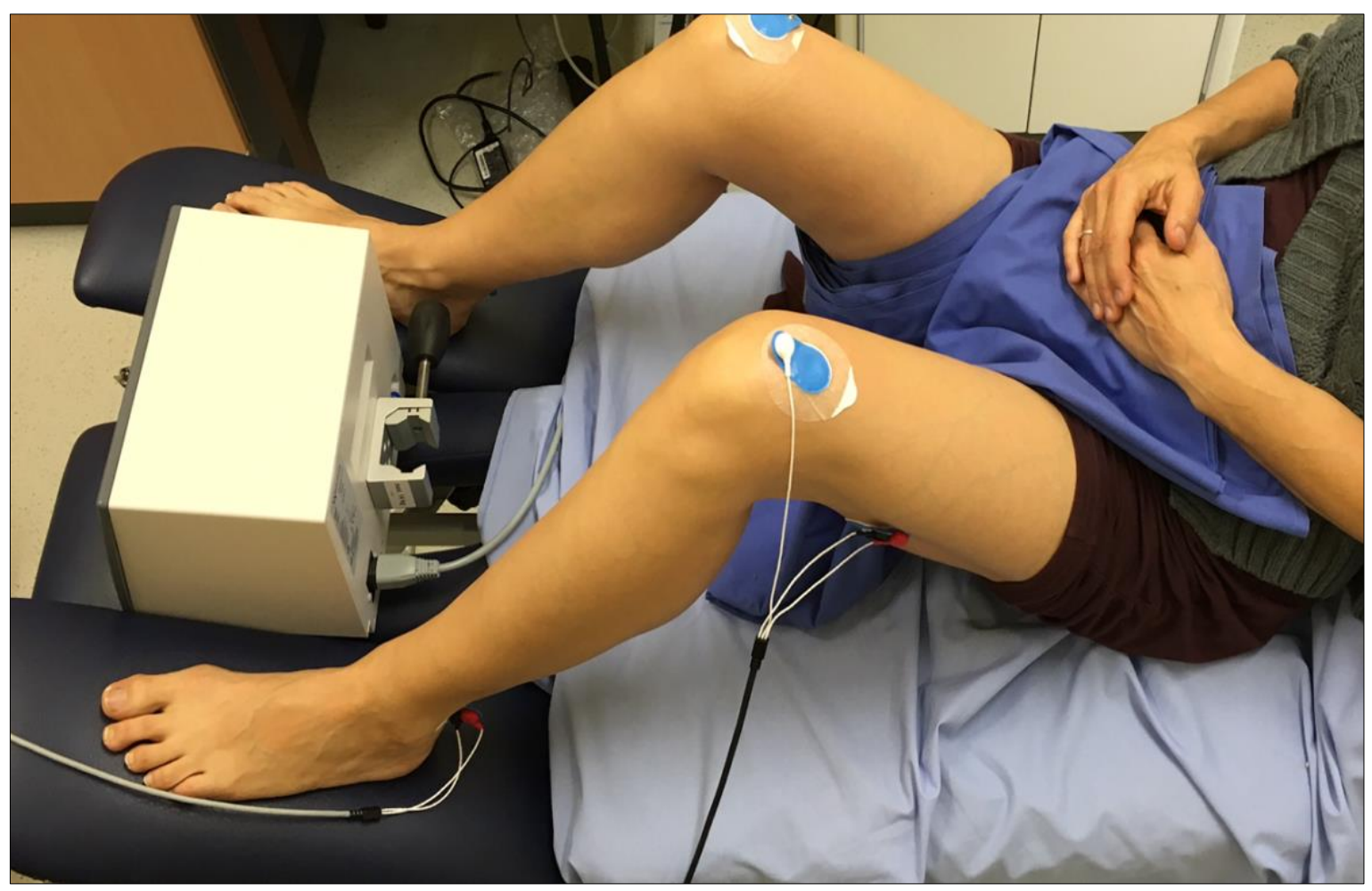

Abbildung 7: Position der Reiz- und Messelektroden beispielhaft am linken Bein

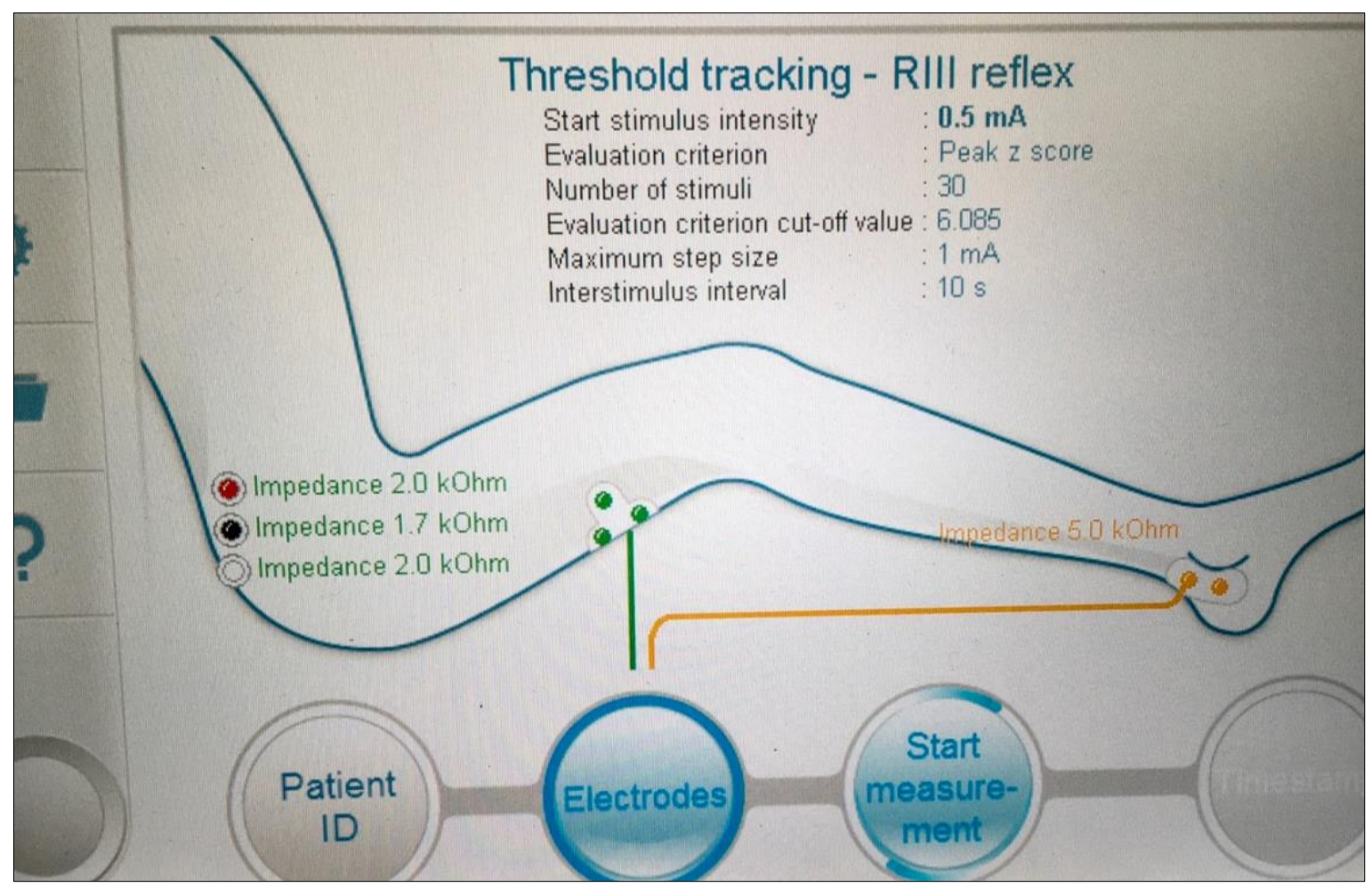

Abbildung 8: Display des Paintracker vor Beginn einer Messung 


\subsection{Biometrie und Datenanalyse}

Statistische Analysen wurden mit dem Programm SPSS-Statistics Version 23 for Mac (IBM Corporation, USA) durchgeführt. Die Ergebnisse sind als Mittelwert (MW) und Standardabweichung (SD) angegeben, das Signifikanzniveau wurde auf alpha $=0,05$ festgelegt. Zum Zeitpunkt der Studiendurchführung gab es nur wenig vergleichbare Studien, sodass die Stichprobengröße der beiden Gruppen an eine Studie für Knie-Arthrose (Kavchak et al. 2012) angelehnt wurde und zusätzlich eine Effektstärkenberechnung erfolgte. Die Stichprobengröße der Probandengruppe wurde daher auf 30 festgelegt (Cohen's d 0,65; Power 0,81). Die insgesamt 18 Probanden der Kontrollgruppe wurden bezüglich der Kriterien Alter und Geschlecht auf die Probanden mit Hüftarthrose, gematcht". Jeder von Koxarthrose betroffenen rechten oder linken unteren Extremität wurde eine passende Extremität bei einem ähnlich alten, gleichgeschlechtlichen Kontrollprobanden zugeordnet. Daher kommt es im weiteren Verlauf der Arbeit zu der Begrifflichkeit der „betroffenen Seite“ bei gesunden Teilnehmern der Studie. In diesem Fall handelt es sich um die Beine der gesunden Teilnehmer der Kontrollgruppe, die jeweils auf schmerzhafte Beine der Probanden ,gematcht"wurden.

Eine Normalverteilung der Daten konnte mithilfe des Shapiro-Wilk-Test für die Werte der Fragebögen und Druckschmerzschwellen, nicht aber für die Schmerzausdehnung und Temperaturschwellenwerte nachgewiesen werden. Gruppenunterschiede zwischen den unabhängigen Stichproben wurden daher für die normalverteilten Daten mit Hilfe zweiseitiger T-Tests und für die nicht normalverteilten Daten mit dem Mann-Whitney-U-Test evaluiert. In einem zweiten Schritt wurde eine two-way mixed-ANOVA verwendet, um mögliche Interaktionen von unabhängigen Variablen wie Geschlecht, Alter oder BMI auf den gemessenen Gruppenunterschied zu zeigen. Die nötigen Voraussetzungen (annähernde Normalverteilung, das Fehlen von extremen statistischen Ausreißern und eine nicht verletzte Sphärizität (MauchlyTest bei nur zwei Stufen nicht relevant)) waren hierfür allesamt erfüllt. Im Fall einer signifikanten ANOVA wären Bonferroni-adjusted post hoc Tests durchgeführt worden.

Korrelationen zwischen Ergebnissen der QST und anderen Parametern wurden bei normalverteilten Daten mit dem Pearson Korrelationskoeffizienten $r$ und bei nicht normalverteilten Daten anhand des Spearman-Rho @ bestimmt. Für einzelne Korrelationen wurde zusätzlich ein möglicher Einfluss von Drittvariablen wie Geschlecht, BMI oder Alter über die Verwendung einer partiellen Korrelationsanalyse untersucht. 


\section{$3 \quad$ Ergebnisse}

\subsection{Klinische Charakteristika der Gruppen}

Alle 48 rekrutierten Probanden absolvierten den vollen Umfang der Studie. Die Geschlechterverteilung betrug sowohl in der Gruppe der Probanden mit Hüftarthrose ( $\mathrm{n}=30$, 15 Frauen und 15 Männer) als auch der Gesunden (n=18, 9 Frauen und 9 Männer) jeweils 50 \%. Die Hälfte der Probanden (8 Männer, 7 Frauen) beschrieben prinzipiell eine bilaterale Koxarthrose, wobei sie eine Seite als deutlich stärker schmerzhaft und klinisch führend angaben. Ein signifikanter Unterschied bezüglich der demographischen Daten zwischen beiden Gruppen konnte weder für das Alter $(\mathrm{p}=0,18)$ noch für den Body Mass Index $(\mathrm{p}=0,30)$ oder die gemessenen Blutdruckwerte ( $\mathrm{p}=0,48$ bzw. $\mathrm{p}=0,60)$ gefunden werden (Tabelle 3). Durchschnittlich gaben die Probanden der Gruppe mit Koxarthrose auf der numerischen Analogskala einen Wert von 4,5 von 10 möglichen Punkten für den Zeitraum der letzten vier Wochen an, wobei der Wert 0 keinem Schmerz und der Wert 10 dem größtmöglich vorstellbaren Schmerz entspricht.

\begin{tabular}{llllll}
\hline & \multicolumn{2}{l}{ Koxarthrose, (n=30) } & \multicolumn{4}{l}{ Kontrollen, (n=18) } \\
\hline Merkmal & MW & SD & MW & SD & p* \\
\hline Alter (Jahre) & 60,3 & \pm 6 & 62,5 & \pm 3 & 1 \\
Männer/ Frauen & $15 / 15$ & - & $9 / 9$ & & - \\
Dauer der Arthrose (Jahre) & 7,2 & \pm 8 & - & & 0,48 \\
Systolischer Blutdruck (mmHG) & 136 & \pm 18 & 139 & \pm 15 & 0,60 \\
Diastolischer Blutdruck (mmHG) & 84 & \pm 8 & 86 & \pm 12 & 0,30 \\
Body Mass Index (kg/m2) & 25 & \pm 4 & 24 & \pm 4 & - \\
Durchschnittlicher Schmerz letzter Monat & 4,5 & \pm 2 & - & & - \\
Oxford Hip Score (x/60) & 29,5 & \pm 7 & - & & - \\
Von Korff-Grad & 1,7 & \pm 1 & - & & - \\
SF-36 Mental Component Scale (MCS) & 50,4 & \pm 7 & - & & - \\
SF-36 Physical Component Scale (PCS) & 36,2 & \pm 8 & - & & \\
\hline
\end{tabular}

Tabelle 3: Klinische Charakteristika der Gruppen *T-Test, Gruppenvergleich 


\subsection{Schmerzcharakteristik der Gruppe mit Koxarthrose}

Wie Abbildung 9 verdeutlich, zeigte das Kollektiv mit chronischer Hüftgelenksarthrose ein heterogenes Spektrum bezüglich der zeitlichen Schmerzcharakteristik. So klagten insgesamt $44 \%$ der Teilnehmer über Schmerzattacken, zwischen denen entweder Schmerzfreiheit herrschte oder nicht und insgesamt 56 \% über Dauerschmerzen.

\section{Zeitliches Schmerzmuster der Patienten mit Koxarthrose, $n=30$}

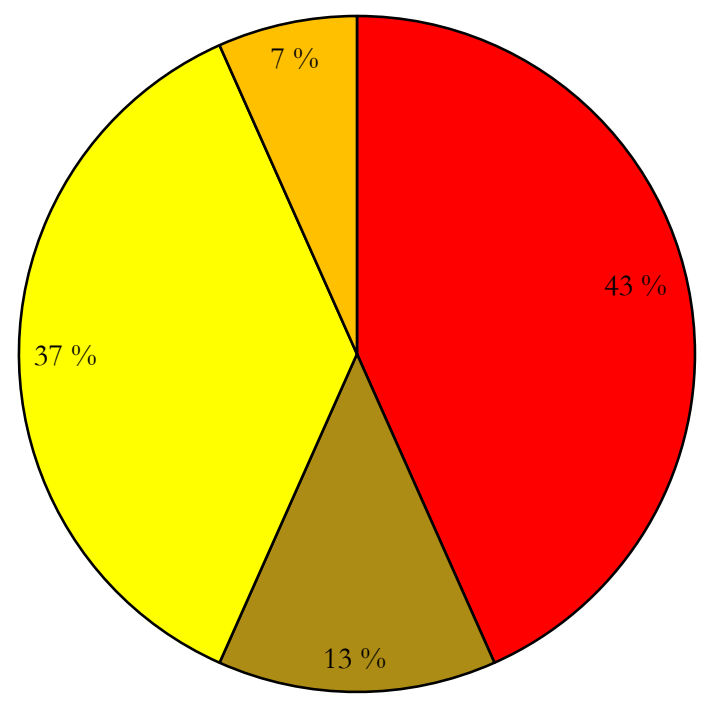

Dauerschmerzen mit leichten Schwankungen

$\square$ Dauerschmerzen mit Schmerzattacken

$\square$ Schmerzattacken dazwischen schmerzfrei

$\square$ Schmerzattacken dazwischen Schmerzen

Abbildung 9: Zeitliches Schmerzmuster der Patienten mit Koxarthrose

Die Auswertung des painDETECT Fragebogens ergab, dass zwei Teilnehmer eine Punktzahl von mehr als 19 Punkten erreichten. Damit erfüllen diese beiden formal die Kriterien für das Vorliegen einer neuropathischen Schmerzcharakteristik (Freynhagen et al. 2006). Da anamnestisch keine Hinweise für ein relevantes neurologisches Krankheitsbild vorlagen, wurde beide Patienten nicht ausgeschlossen. 
Die schmerzbedingte Einschränkung im Alltag wurde mithilfe des Oxford Hip Scores gemessen, der sich im Vergleich zu anderen ähnlichen Fragebögen durch seine hohe Relevanz bei einfacher Handhabung auszeichnet (Naal et al. 2015).

Abbildung 10 zeigt die Verteilung des Oxford Hip Scores unter den 30 Studienteilnehmern mit Hüftarthrose. Etwa $43 \%$ der Teilnehmer erreichten 30 oder mehr Punkte, lediglich ein Teilnehmer erreichte die Marke von 20 Punkten nicht.

Zwischen Frauen und Männern konnte kein signifikanter Unterschied $(p=0,146)$ für die Ergebnisse des Oxford Hip Scores ermittelt werden (Tabelle 4).

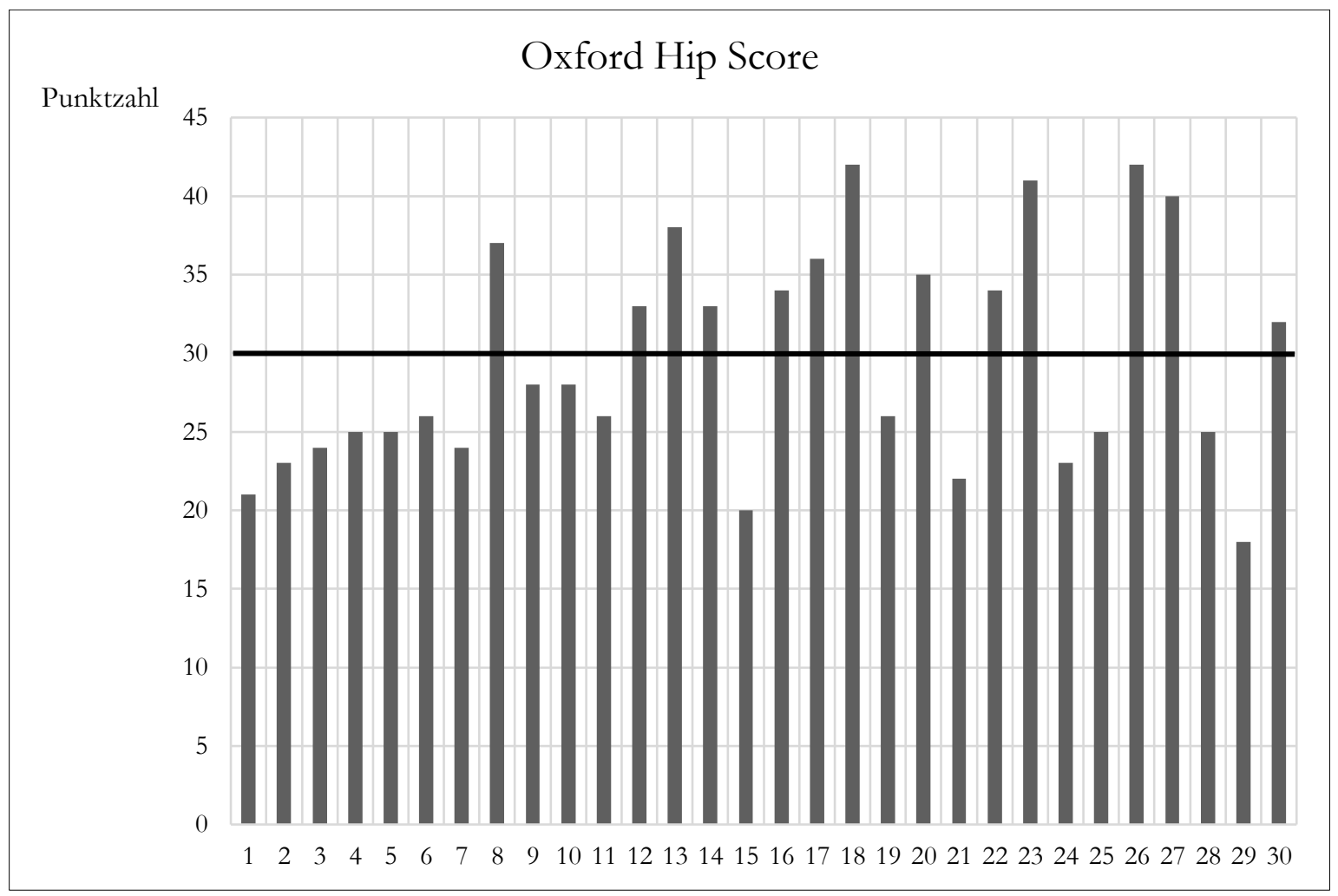

Abbildung 10: Verteilung des Oxford Hip Scores innerhalb der Hüftarthrosegruppe 
Neben dem Grad der hüftbezogenen Einschränkung im Alltag ist die Beeinträchtigung durch die chronischen Schmerzen selber von Interesse. Diese lässt sich mithilfe der weiter oben bereits beschriebenen Schweregradeinteilung nach Von-Korff einschätzen. In unserer Studie erreichten etwa zwei Drittel der Probanden mit chronischer Hüftarthrose einen Von-Korff-Grad I mit geringer schmerzbezogener Beeinträchtigung, während insgesamt nur $20 \%$ der Teilnehmer Grad III oder IV erreichten (Abbildung 11).

Es fanden sich dabei keine signifikanten Unterschiede zwischen beiden Geschlechtern $(\mathrm{p}=0,790)$.

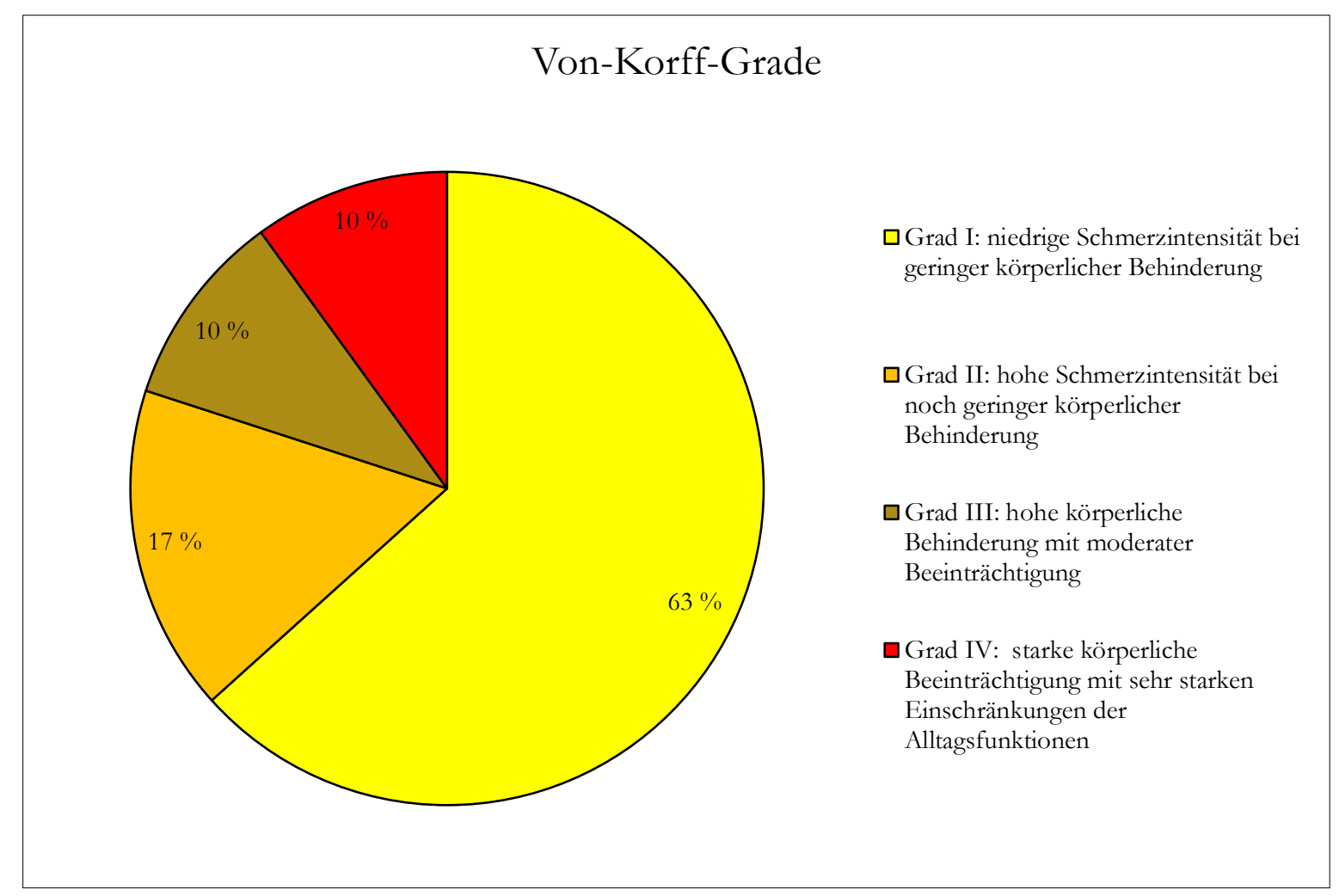

Abbildung 11: Verteilung der Probanden mit Hüftarthrose auf die Von-Korff-Grade 


\subsection{Ergebnisse des Fibromyalgie-Syndrom Survey Questionnaires}

Insgesamt etwa $63 \%$ der Probanden der Gruppe mit Koxarthrose gaben mehr als drei schmerzempfindliche Regionen des Körpers im Widespread Pain Index (WPI) an, einer Subskala des Fibromyalgie-Syndrom Survey-Questionnaires.

Ein signifikanter Geschlechtsunterschied konnte für den WPI allerdings nicht nachgewiesen werden $(p=0,935)$. Neben den zu erwartenden Schmerzen im Bereich der Hüftgelenke und des Oberschenkels gaben $47 \%$ der Probanden zusätzlich Schmerzen in einer oder beiden Schultergelenken, $57 \%$ im Bereich des unteren Rückens sowie $20 \%$ der Teilnehmer zusätzliche Schmerzen im Bereich des Nackens bzw. des Unterschenkels an.

Allerdings erreichten lediglich $14 \%$ der Teilnehmer sechs oder mehr Punkte in der zweiten Subskala (Symptom Severity Score, SSS). Auch hier fanden sich keine signifikanten Geschlechtsunterschiede $(\mathrm{p}=0,412)$.

Die vollen Kriterien des Fragebogens wurden somit nur von drei Teilnehmern erreicht und legen differentialdiagnostisch den Verdacht auf ein mögliches Fibromyalgie-Syndrom nahe. Allerdings kann mit dem Survey allein nicht die klinische Diagnose gestellt werden, sodass die Teilnehmer in der Gesamtauswertung berücksichtigt wurden. Neben dem oben genannten Widespread Pain Index fand in unserer Studie auch die Auswertung der digitalen Schmerzkarten zur Diagnostik einer möglichen generalisierten Schmerzempfindung Verwendung.

\subsection{Ergebnisse der Schmerzausdehnung}

Zwischen der Schmerzausdehnung bei Frauen (Abbildung 12) und der bei Männern (Abbildung 13) ergaben sich signifikante Unterschiede $(\mathrm{p}<0,01)$ hinsichtlich der Ausdehnung der markierten Pixelfläche.

Männer gaben im Mittel 2,65 \% der markierten Körperfläche im Diagramm als schmerzhaft an, während Frauen durchschnittlich 6,71 \% der Fläche markierten.

Graphisch fällt auf, dass bei beiden Geschlechtern neben Beschwerden im Bereich der jeweilig betroffenen Hüfte auch Markierungen der Gegenseite und anderer gelenkferner Regionen getroffen wurden. Besonders häufig markierte Schmerzorte werden dem Farbverlauf hier entsprechend dunkler dargestellt. 


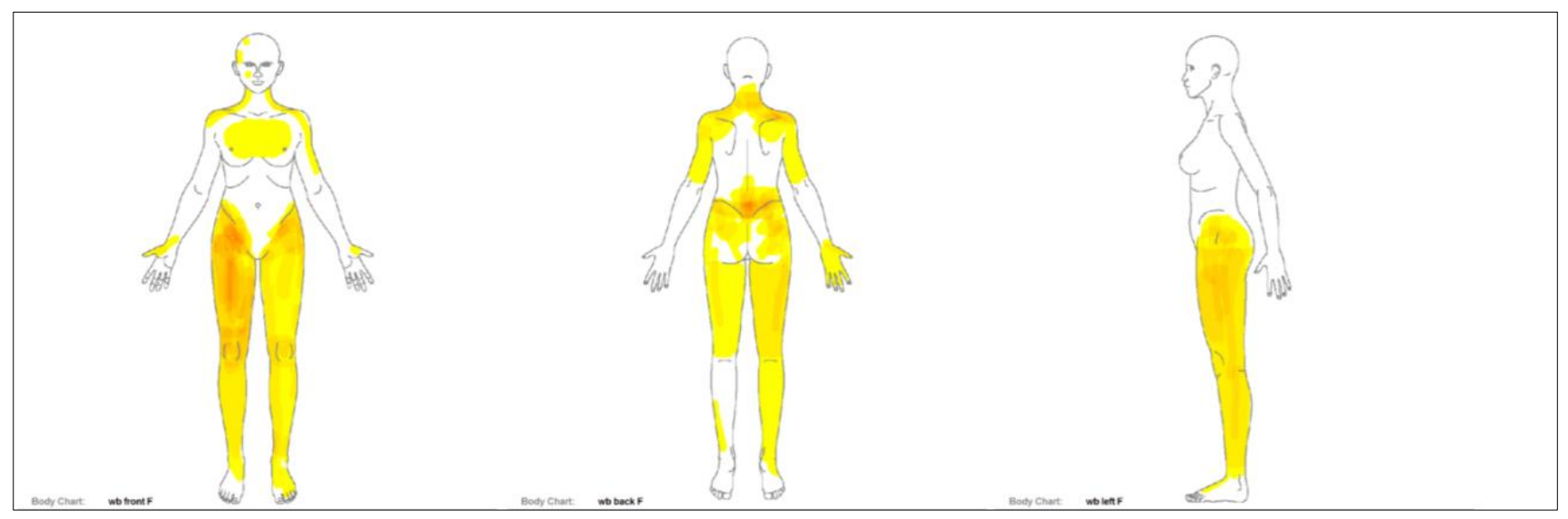

Abbildung 12: Ergebnisse der Schmerzkarten bei weiblichen Probanden

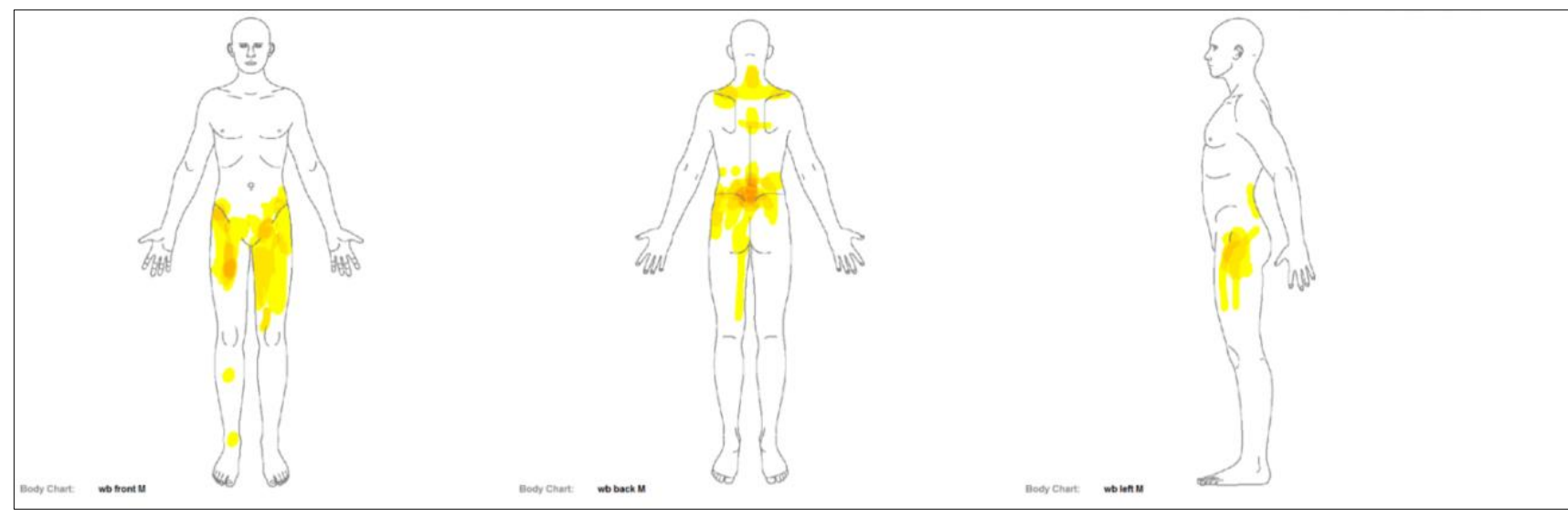

Abbildung 13: Ergebnisse der Schmerzkarten bei männlichen Probanden 


\subsection{Ergebnisse der psychologischen Fragebögen}

Die Probanden der Gruppe mit Koxarthrose erreichten bezüglich der weiteren Fragebögen (PCS, CPAQ, DASS, TSK-DE) durchschnittliche Werte, die keine entsprechenden Grenzwerte erreichten (Tabelle 4). Bezüglich der krankheitsbedingt eingeschränkten Lebensqualität (SF-36 Health Survey) der Probanden in unserer Studie wurden im Vergleich zu deutschlandweiten Erhebungen (Ellert und Kurth 2013) signifikant erniedrigte Werte für die körperliche Achse (PCS; $\mathrm{p}<0.0001$ ), aber nicht für die psychische Achse (MCS; $\mathrm{p}>0,05)$ gemessen. Für keinen der ausgewerteten Fragebögen fiel ein signifikanter Geschlechtsunterschied auf (Tabelle 4).

\begin{tabular}{|c|c|c|c|c|c|c|c|c|c|}
\hline \multirow[b]{2}{*}{ Fragebogen } & \multicolumn{4}{|c|}{$\begin{array}{l}\text { Gesamt } \\
(\mathrm{n}=30)\end{array}$} & \multicolumn{2}{|c|}{$\begin{array}{l}\text { Männer } \\
(\mathrm{n}=15)\end{array}$} & \multicolumn{2}{|c|}{$\begin{array}{l}\text { Frauen } \\
(\mathrm{n}=15)\end{array}$} & \multirow[b]{2}{*}{$\mathrm{p}^{*}$} \\
\hline & MW & SD & Min. & Max. & MW & $\mathrm{SD}$ & MW & $\mathrm{SD}$ & \\
\hline Oxford Hip Score & 29,5 & 7,1 & 18,0 & 42,0 & 27,4 & 5,5 & 31,7 & 8,0 & 0,146 \\
\hline Von-Korff-Grad & 1,7 & 1,0 & 1,0 & 4,0 & 1,1 & 1,6 & 1,9 & 1,6 & 0,129 \\
\hline SF-36 Physical Component Scale (PCS) & 36,2 & 6,6 & 22,3 & 48,1 & 39,3 & 5,4 & 34,7 & 7,0 & 0,468 \\
\hline SF-36 Mental Component Scale (MCS) & 50,4 & 8,1 & 29,8 & 63,7 & 49,0 & 7,9 & 50,6 & 9,3 & 0,787 \\
\hline $\begin{array}{l}\text { Fibromyalgie Survey Criteria } \\
\text { (Symptom Severity Score) }\end{array}$ & 3,4 & 2,3 & 0,0 & 9,0 & 3,1 & 2,2 & 3,7 & 2,5 & 0,390 \\
\hline $\begin{array}{l}\text { Fibromyalgie Survey Criteria } \\
\text { (Wide Spread Pain Index) }\end{array}$ & 3,9 & 1,8 & 1,0 & 8,0 & 3,9 & 1,5 & 4,1 & 2,1 & 0,915 \\
\hline Pain Acceptance Questionnaire (total) & 63,9 & 9,2 & 47,0 & 85,0 & 63,2 & 9,5 & 64,7 & 9,1 & 0,480 \\
\hline PainDETECT Score & 8,4 & 4,9 & 0,0 & 21,0 & 8,3 & 5,3 & 8,6 & 4,7 & 0,770 \\
\hline $\begin{array}{l}\text { Durchschnittlicher Schmerz im letzten Monat } \\
\text { (NRS) }\end{array}$ & 4,5 & 2,2 & 1,0 & 8,0 & 4,1 & 2,2 & 4,9 & 2,1 & 0,377 \\
\hline Pain Catastrophizing Scale (total) & 12,4 & 11,3 & 0,0 & 44,0 & 10,3 & 9,8 & 14,5 & 12,6 & 0,360 \\
\hline Tampa Scale for Kinesiophobia (TSK-DE) & 35,1 & 6,0 & 26,0 & 54,0 & 36,7 & 6,7 & 33,5 & 5,0 & 0,220 \\
\hline $\begin{array}{l}\text { Depression Anxiety Stress Scale (DASS) } \\
\text { (Depression) }\end{array}$ & 3,1 & 4,0 & 0,0 & 17,0 & 2,5 & 3,2 & 3,7 & 4,7 & 0,554 \\
\hline $\begin{array}{l}\text { Depression Anxiety Stress Scale (DASS) } \\
\text { (Stress) }\end{array}$ & 3,8 & 3,6 & 0,0 & 14,0 & 3,5 & 3,7 & 4,1 & 3,5 & 0,517 \\
\hline $\begin{array}{l}\text { Depression Anxiety Stress Scale (DASS) } \\
\text { (Anxiety) }\end{array}$ & 1,6 & 2,2 & 0,0 & 10,0 & 1,1 & 1,2 & 2,1 & 1,3 & 0,630 \\
\hline
\end{tabular}

Tabelle 4: Ergebnisse der Fragebögen

*T-Test Geschlechtsunterschied 


\subsection{Ergebnisse der Quantitativ-Sensorischen Testung}

\subsubsection{Gruppenunterschiede}

Für die thermalen Schwellenwerte fanden sich lediglich für die Parameter CPT am Thenar der Hand der nicht betroffenen Seite $(\mathrm{p}<0,018)$ und WDT am betroffenen Trochanter major $(\mathrm{p}<0,021)$ statistisch signifikante Unterschiede (Tabelle 5).

\begin{tabular}{lccccc}
\hline & Koxarthrose $(\mathrm{n}=30)$ & \multicolumn{2}{c}{ Kontrollen (n=18) } \\
\hline & Mittelwert & SD & Mittelwert & SD & $\mathbf{p}^{*}$ \\
Nicht betroffene Seite & & & & \\
CPT $\left({ }^{\circ} \mathrm{C}\right)$ Thenar & 20,8 & 9,8 & 13,4 & 7,7 & $\mathbf{0 , 0 1 8}$ \\
PPT $(\mathrm{kPa})$ Trochanter major & 418 & 146 & 600 & 122 & $\mathbf{0 , 0 0 0}$ \\
PPT $(\mathrm{kPa})$ M. gluteus medius & 421 & 142 & 557 & 157 & $\mathbf{0 , 0 0 6}$ \\
PPT $(\mathrm{kPa})$ M. tibialis anterior & 408 & 141 & 547 & 138 & $\mathbf{0 , 0 0 1}$ \\
PPT $(\mathrm{kPa})$ Thenar & 361 & 106 & 494 & 88 & $\mathbf{0 , 0 0 0}$ \\
Betroffene Seite & & & 35,1 & 1,2 & $\mathbf{0 , 0 2 1}$ \\
WDT $\left({ }^{\circ} \mathrm{C}\right)$ Trochanter major & 37,4 & 3,8 & 591 & 140 & $\mathbf{0 , 0 0 0}$ \\
PPT $(\mathrm{kPa})$ Trochanter major & 400 & 149 & 544 & 153 & $\mathbf{0 , 0 0 1}$ \\
PPT $(\mathrm{kPa})$ M. gluteus medius & 384 & 134 & 547 & 140 & $\mathbf{0 , 0 0 2}$ \\
PPT $(\mathrm{kPa})$ M. tibialis anterior & 394 & 130 & 482 & 100 & $\mathbf{0 , 0 0 0}$ \\
PPT $(\mathrm{kPa})$ Thenar & 355 & 110 & & & \\
\hline
\end{tabular}

Tabelle 5: Übersicht der statistisch signifikanten QST-Gruppenunterschiede, $C P T=$ Cold Pain Threshold, PPT =Pressure Pain Threshold, WDT $=W$ arm Detection Threshold

* T-Test: PPT Gruppenunterschied, Mann-Whitney U Test: CPT, WDT Gruppenunterschied

Bezüglich der Druckschmerzschwellen wurden signifikant niedrigere Werte bei den an Hüftarthrose Erkrankten gegenüber den Gesunden gemessen. Demnach waren Menschen mit Koxarthrose druckschmerzempfindlicher oberhalb des Trochanter major ( $\mathrm{p}<0,0001)$, M. gluteus medius $(\mathrm{p}<0,001)$, M. tibialis anterior $(\mathrm{p}<0,002)$ und des Thenars $(\mathrm{p}<0,0001)$.

Zusätzlich wurden auch kontralateral, also über der eigentlich nicht von Arthrose betroffenen Seite, sowohl periartikulär (Abbildung 15, p<0,001) als auch gelenkfern (Abbildung 14, $\mathrm{p}<0,003)$ niedrigere Werte für Druckschmerz in der Arthrose-Gruppe gemessen. Im Bereich der Mm. vastus medialis und lateralis ergaben sich keine signifikanten Gruppenunterschiede, sodass diese für die weitere graphische Darstellung keine Verwendung finden. 


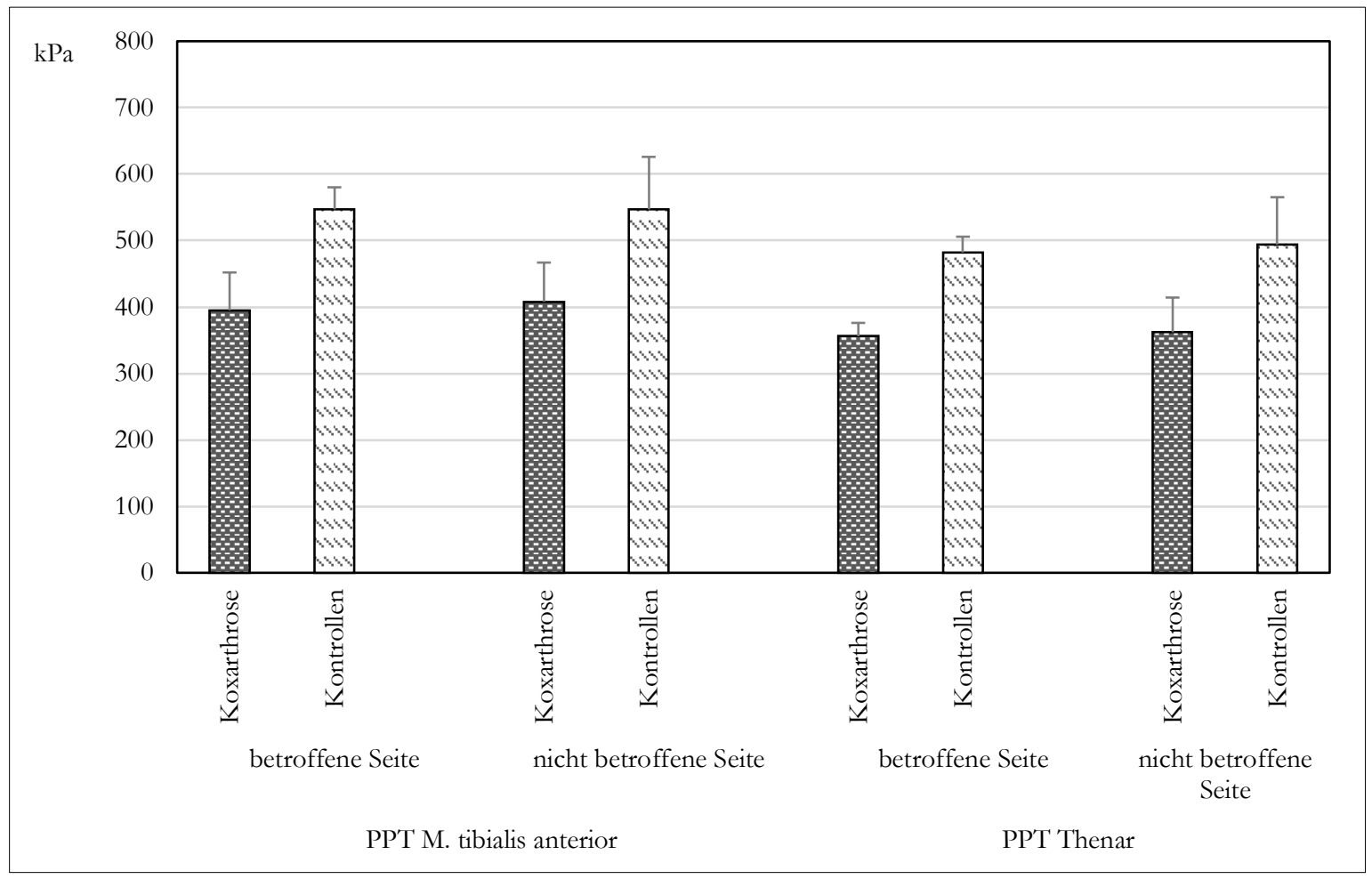

Abbildung 14: Hüftgelenksferne Druckschmerzschwellen (M. tibialis anterior und Thenar) im Gruppenvergleich, alle Unterschiede signifikant, statistische Werte siehe Tabelle 5, PPT $=$ Pressure Pain Threshold

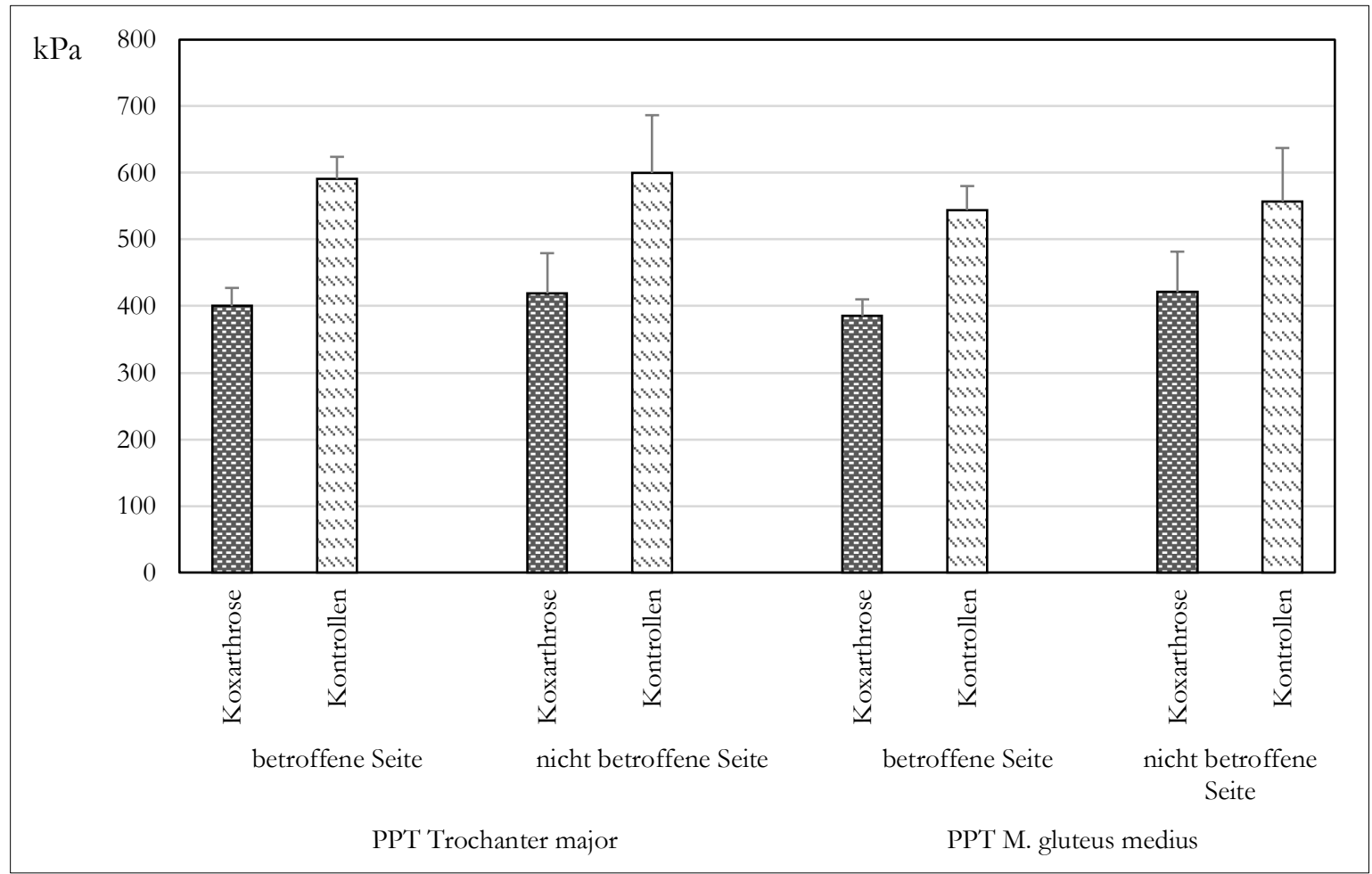

Abbildung 15: Hüftgelenksnahe Druckschmerzschwellen (Trochanter major, M. gluteus medius) im Gruppenvergleich, alle Unterschiede signifikant, statistische Werte siehe Tabelle 5, PPT $=$ Pressure Pain Threshold 


\subsubsection{Unterschiede zwischen betroffener und nicht betroffener Seite}

Innerhalb der Gruppe mit Koxarthrose ergaben sich zwischen der von Hüftarthrose betroffenen Seite und der nicht bzw. geringer betroffenen Seite signifikante Gruppenunterschiede. So waren die Patienten bezüglich Kälteschmerz (CPT; p<0,001) an der betroffenen Hüfte, aber auch bezüglich Hitzeschmerz (HPT; p<0,010) und Kälteschmerz (CPT; $\mathrm{p}<0,006)$ am Thenar der betroffenen Seite empfindlicher als auf der Gegenseite. Betreffend Druckschmerz fanden sich vor allem oberhalb des betroffenen M. gluteus medius signifikant niedrigere Schwellen ( $<<0,009)$ im Seitenvergleich (Tabelle 7 im Anhang).

\subsubsection{Geschlechtsunterschiede}

Für die Ergebnisse der Quantitativ-Sensorischen Testung ergaben sich signifikante Unterschiede $(p<0,05)$ zwischen Männern und Frauen. Innerhalb der Gruppe mit Hüftarthrose waren Frauen insgesamt druckschmerzempfindlicher als Männer (Tabelle 9 im Anhang) sowohl an hüftgelenksnahen (Abbildung 17) als auch an peripheren (Abbildung 16) Messorten.

Allerdings waren auch innerhalb der Kontrollgruppe Frauen signifikant druckschmerzempfindlicher als Männer (Tabelle 10 im Anhang). Beispielsweise war dies oberhalb des M. gluteus medius der betroffenen $(p<0,003)$ und nicht betroffenen Seite $(p<0,0001)$, sowie oberhalb des M. tibialis anterior der betroffenen Seite $(\mathrm{p}<0,025)$ messbar. Für die restlichen Messorte wurden statistische Tendenzen gemessen $(p>0,05)$.

Um einen möglichen Einfluss dieses Unterschiedes auf den gemessenen Gruppenunterschied für Druckschmerz zu untersuchen, führten wir eine two-way mixed $A N O V A$ durch. Diese ergab signifikante Gruppenunterschiede $(\mathrm{F}=19,31 ; \mathrm{p}<0,0001)$ und Geschlechtsunterschiede $(\mathrm{F}=11,20 ; \mathrm{p}<0,01)$, allerdings keine signifikante Interaktion zwischen diesen beiden Variablen $(\mathrm{F}(1,46)=0,31 ; \mathrm{p}=0,581)$ für Druckschmerz in hüftnahen Regionen. Auch für periphere Messorte wurden zwar signifikante Gruppenunterschiede $(\mathrm{F}=23,37 ; \mathrm{p}<0,0001)$ und Geschlechtsunterschiede $(F=8,90 ; \mathrm{p}<0,005)$, jedoch keine signifikante Interaktion zwischen diesen gemessen $(\mathrm{F}(1,46)=0,003 ; \mathrm{p}=0,957)$.

Bezüglich des BMI gab es keine signifikanten Unterschiede zwischen den Geschlechtern sowohl in der Gruppe mit Koxarthrose $(p=0,106)$ als auch in der Kontrollgruppe $(p=0,113)$. Auch für die Werte des systolischen Blutdrucks innerhalb der Koxarthrosegruppe $(p=0,148)$ und der Kontrollgruppe $(p=0,077)$, sowie für das Alter ( $p=0,436$ bzw. $p=0,258)$ wurden keine signifikanten Geschlechtsunterschiede festgestellt. Die two-way mixed ANOVA zeigte bezüglich eines möglichen Einflusses der Variable BMI auf hüftgelenksnahe Druckschmerzschwellen ein knapp nicht signifikantes Ergebnis $(F(14,33)=1,8 ; p=0,081)$, während ein potentieller Einfluss des BMI auf hüftgelenksferne Druckschmerzschwellen statistisch nicht nachgewiesen werden 
konnte $(\mathrm{F}(14,33)=0,79 ; \mathrm{p}=0,676)$. Auch für das Alter als mögliche beeinflussende Variable wurde sowohl hüftgelenksfern $F(18,29)=1,27 ; p=0,278)$ als auch gelenknah $(F(14,28)=1,11$; $\mathrm{p}=0,393)$ kein signifikanter Einfluss gemessen.

Beide Geschlechter mit Koxarthrose gaben zusammen durchschnittlich in den letzten vier Wochen einen Wert von 4,5/10 auf der numerischen Schmerzskala an, es bestand auch hier kein signifikanter Unterschied zwischen den Geschlechtern ( $\mathrm{p}=0,461)$. Wie bereits erwähnt, ergaben sich für die Gesamtfläche der Schmerzausdehnung in den digitalen Karten signifikante Geschlechtsunterschiede $(\mathrm{p}<0,01)$. Frauen gaben hier eine deutlich größere Fläche an als die männlichen Teilnehmer.

Auch für die thermischen Schwellenwerte fanden sich signifikante Geschlechtsunterschiede. Diese sind für die Gruppe mit Hüftarthrose in der Abbildung 18 und der Abbildung 19 gezeigt. Für die Kontrollgruppe ergaben sich hier keine signifikanten Unterschiede ( $p>0,05)$. 


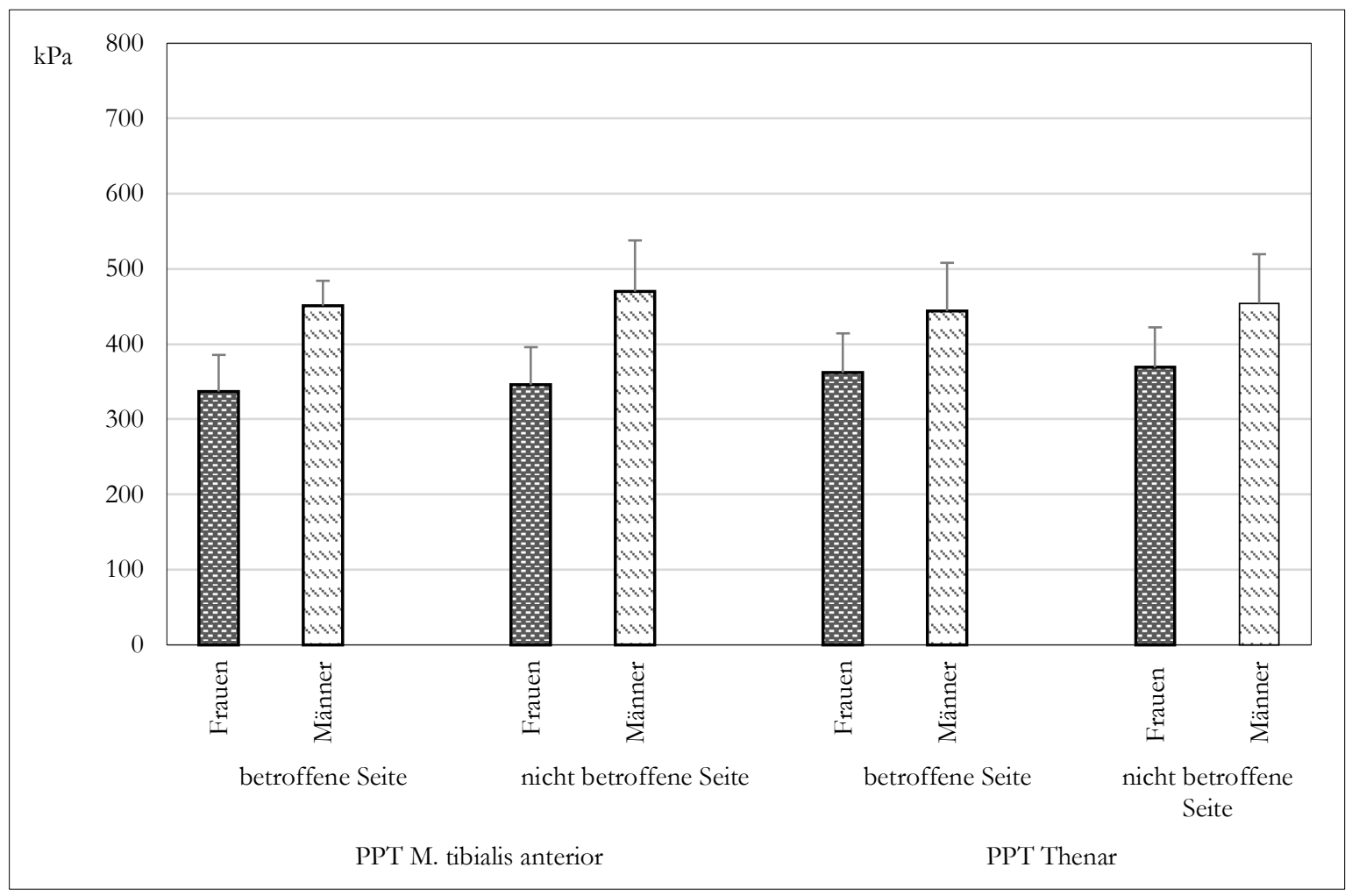

Abbildung 16: Hüftgelenksferne Druckschmerzschwellen (M. tibialis anterior und Thenar) im Vergleich zwischen Frauen und Männern innerhalb der Hüftarthrosegruppe, PPT $=$ Pressure Pain Threshold

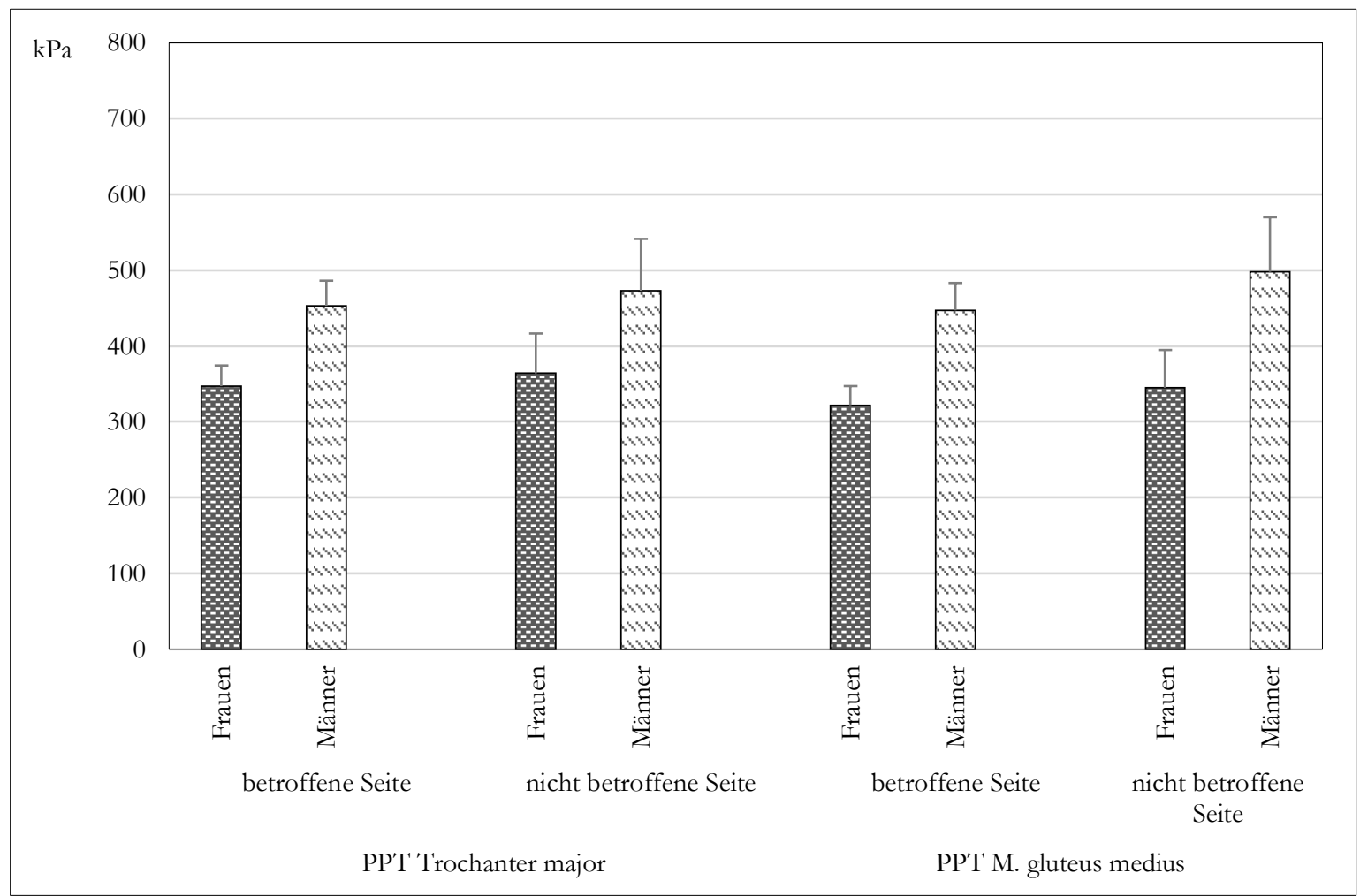

Abbildung 17: Hüftgelenksnahe Druckschmerzschwellen (Trochanter major und M. gluteus medius) im Vergleich zwischen Frauen und Männern innerhalb der Hüftarthrosegruppe, PPT $=$ Pressure Pain Threshold 


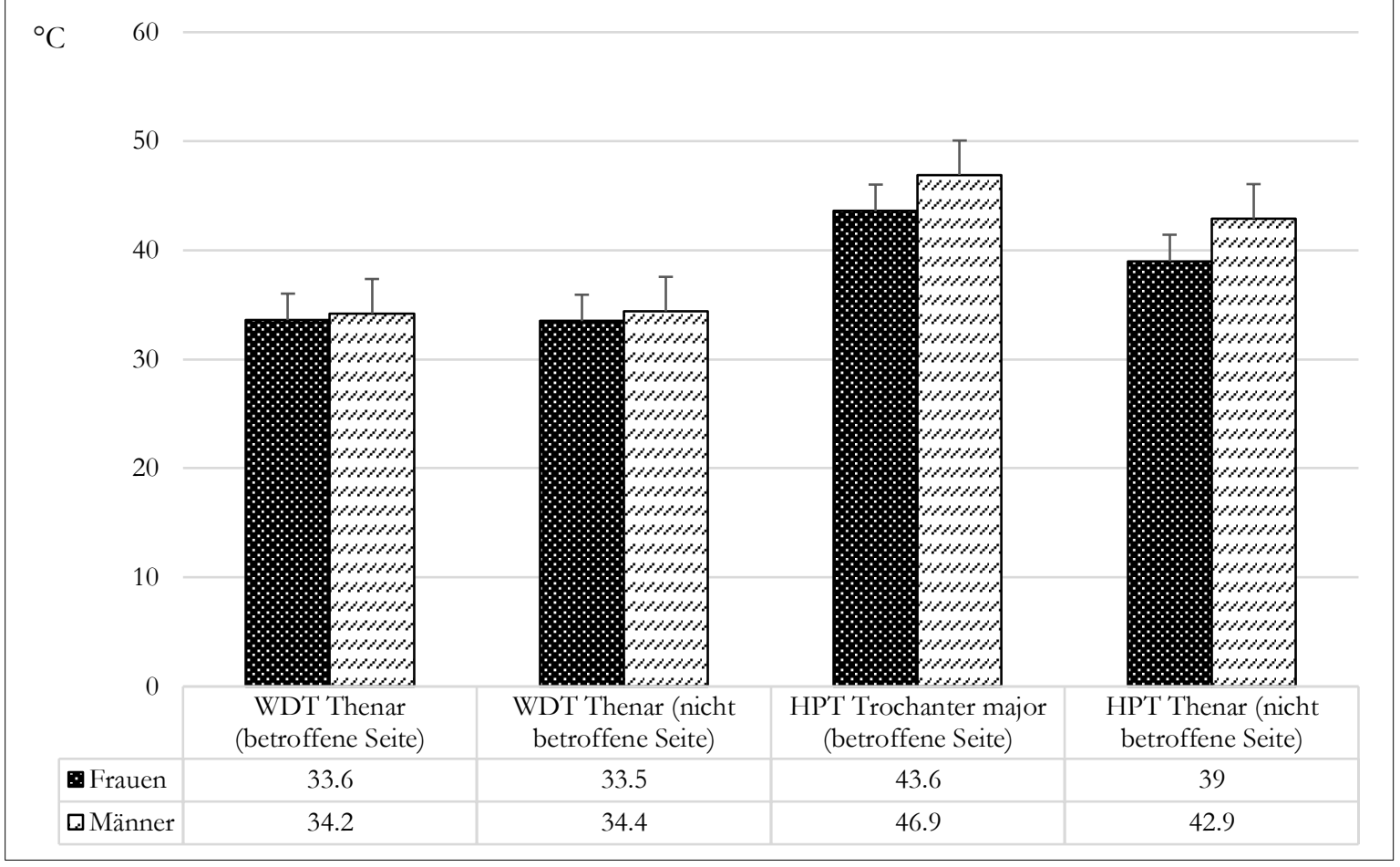

Abbildung 18: Wärmedetektions- (WDT) und -Wärmeschmerzschwellen (HPT) innerhalb der Gruppe mit Hüftarthrose

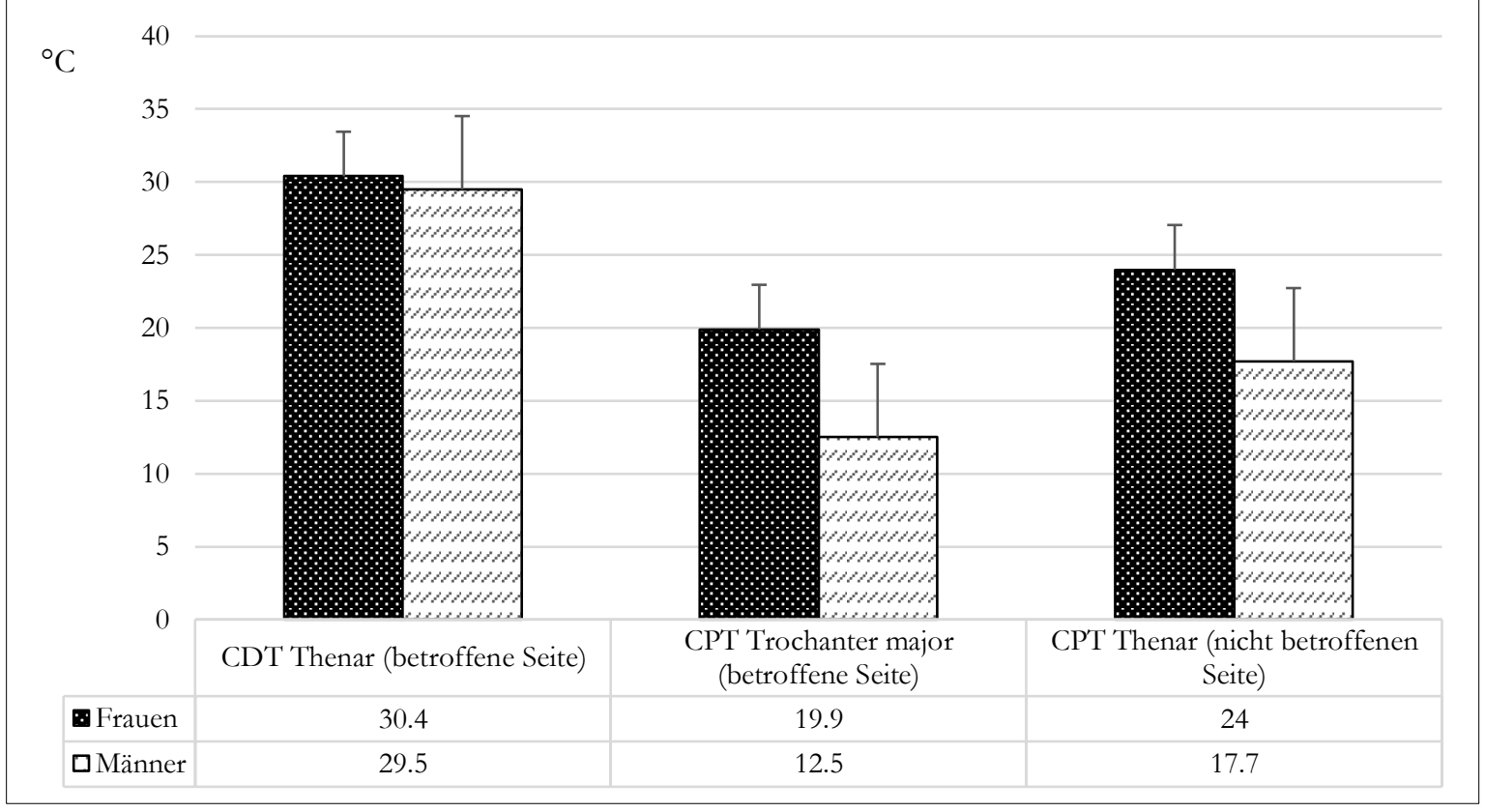

Abbildung 19: Kältedetektions- (CDT) und Kälteschmerzschwellen (CP') innerhalb der Gruppe mit Hüftarthrose 


\subsection{Korrelationen zwischen verschiedenen Mess-Parametern}

\subsubsection{Korrelationen zwischen der Schmerzausdehnung und Ergebnissen der}

\section{Fragebögen}

Die in den digitalen Schmerzkarten erhobene Gesamtfläche der Schmerzausdehnung korrelierte signifikant mit dem WPI ( $\mathrm{r}=0,426, \mathrm{p}<0,05)$, dem painDETECT $(\mathrm{r}=0,394, \mathrm{p}<0,05)$ und dem Pain Catastrophizing Scale $(\mathrm{r}=0,413, \mathrm{p}<0,05)$. Für die anderen Fragebögen konnte kein signifikanter Zusammenhang mit den Ergebnissen der digitalen Schmerzkarten gezeigt werden.

\subsubsection{Korrelationen zwischen der Schmerzausdehnung und Ergebnissen der QST}

Es zeigten sich signifikante Korrelationen der Schmerzausdehnung mit niedrigeren Druckschmerzschwellen über Thenar $(\mathrm{r}=-0,410, \mathrm{p}<0,05), M m$. vastus lateralis $(\mathrm{r}=-0,530, \mathrm{p}<0,01)$ und medialis $(\mathrm{r}=-0,363, \mathrm{p}<0,05)$ sowie Trochanter major $(\mathrm{r}=0,503, \mathrm{p}<0,01)$. Weiterhin konnte die Schmerzausdehnung mit einem erhöhten Cold Pain Threshold $(\mathrm{r}=0,503, \mathrm{p}<0,01)$ und erniedrigten Heat Pain Threshold $(\mathrm{r}=-0,382, \mathrm{p}<0,05)$ über dem Trochanter major, sowie einem erniedrigten Warm Detection Threshold über dem Thenar $(\mathrm{r}=-0,390, \mathrm{p}<0,05)$ korreliert werden.

\subsubsection{Korrelationen zwischen der Druckschmerzempfindlichkeit und weiteren} Variablen

Mithilfe des Korrelationskoeffizienten nach Pearson konnte ein signifikanter Zusammenhang zwischen erhöhten Werten im Oxford Hip Score (=schlechtere Funktion) und niedrigeren Druckschmerzschwellen sowohl über hüftgelenksassoziierten (Abbildung 20; $r=-0,404$, $\mathrm{p}<0,027$ ) als auch über peripheren Messorten der betroffenen Seite (Abbildung 21; $r=-0,412$, $\mathrm{p}<0,024)$ gefunden werden. 


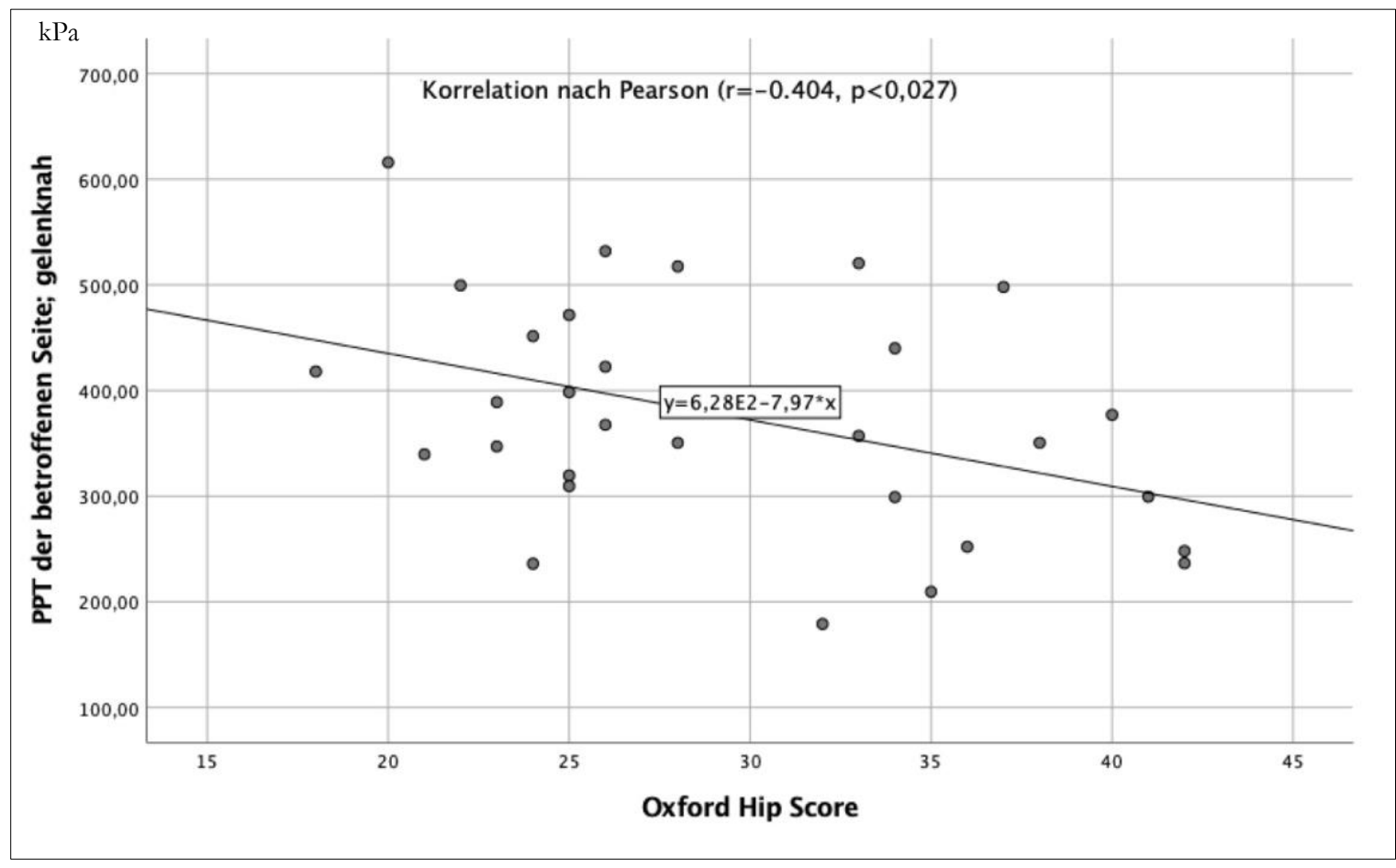

Abbildung 20: Korrelation zwischen Oxford Hip Score und hüftgelenksnahem PPT

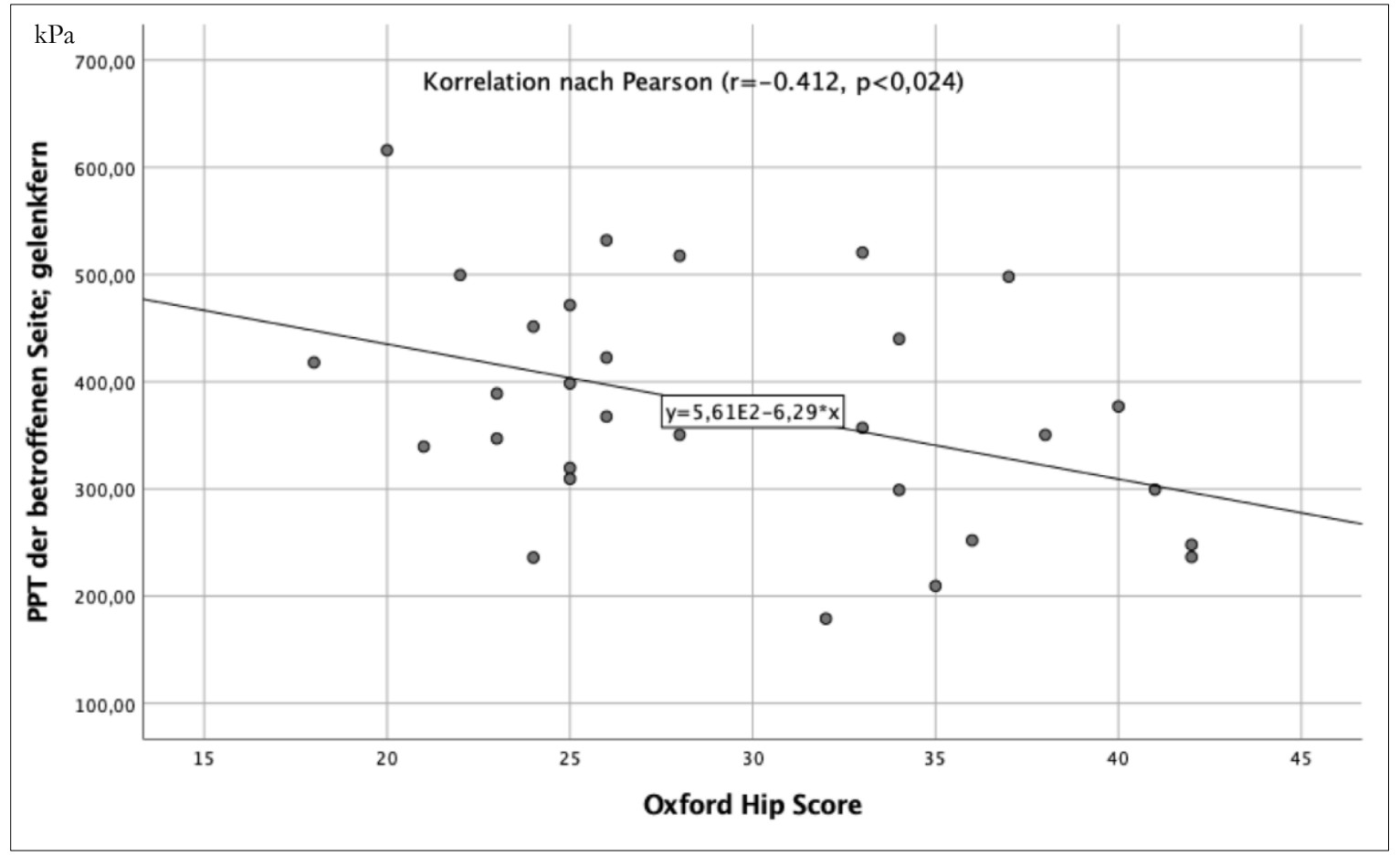

Abbildung 21: Korrelation zwischen Oxford Hip Score und hüftgelenksfernem PPT

Um den Einfluss des Faktor Geschlecht auf die gemessenen Korrelationen zu prüfen wurde zusätzlich eine partielle Korrelationsanalyse mit der Kontrollvariable Geschlecht durchgeführt. Hier zeigte sich eine abgeschwächte, aber noch signifikante Korrelation für den Oxford Hip Score mit hüftgelenksnahen $(\mathrm{r}=-0,318, \mathrm{p}<0,046)$ und hüftgelenksfernen Druckschmerzschwellen der 
betroffenen Seite $(r=-0,316, \mathrm{p}<0,047)$. Für die Faktoren BMI und Alter konnten hier keine signifikanten Einflussfaktoren ( $p>0,05)$ gemessen werden.

Der Body Mass Index korrelierte mit verschiedenen Druckschmerzschwellen über Messpunkten an betroffenen und nicht betroffenen Seiten. Nach der durchgeführten partiellen Korrelation mit Korrektur für den Faktor Geschlecht zeigten sich ebenfalls leicht abgeschwächte, aber noch signifikante Korrelationen (Tabelle 6).

Bezüglich Korrelationen zwischen Druckschmerzschwellen und dem Widespread Pain Index konnten nach partieller Korrelationsanalyse (Korrektur für den Faktor Geschlecht) signifikante Werte für den hüftnahen PPT der nicht betroffenen Seite $(-0,362, \mathrm{p}<0,027)$ und eine statistische Tendenz für den hüftnahen PPT der betroffenen Seite $(-0,304, \mathrm{p}<0,055)$ gemessen werden. Für die weiteren Fragebögen, die neuropathische Schmerzkomponenten (painDETECT) oder psychologische Komorbiditäten (zum Beispiel DASS) detektieren, konnten keine statistisch signifikanten Korrelationen mit Werten aus dem QST nachgewiesen werden.

\begin{tabular}{lcccc}
\hline Pearson & \multicolumn{2}{c}{$\begin{array}{c}\text { Korrelationskoeffizient vor } \\
\text { Korrektur }\end{array}$} & $\begin{array}{c}\text { Korrelationskoeffizient mit Korrektur } \\
\text { für Geschlecht }\end{array}$ \\
\hline Betroffene Seite & $\mathrm{r}=$ & $\mathrm{p}^{*}$ & $\mathrm{r}=$ & $\mathrm{p}^{*}$ \\
\hline PPT Trochanter major & 0,402 & $\mathbf{0 , 0 2 8}$ & 0,344 & $\mathbf{0 , 0 3 4}$ \\
PPT M. gluteus medius & 0,388 & $\mathbf{0 , 0 3 4}$ & 0,315 & $\mathbf{0 , 0 4 8}$ \\
PPT M. vastus medialis & 0,468 & $\mathbf{0 , 0 0 9}$ & 0,419 & $\mathbf{0 , 0 1 2}$ \\
PPT M. vastus lateralis & 0,517 & $\mathbf{0 , 0 0 3}$ & 0,468 & $\mathbf{0 , 0 0 5}$ \\
PPT M. tibialis anterior & 0,518 & $\mathbf{0 , 0 0 3}$ & 0,467 & $\mathbf{0 , 0 0 5}$ \\
Nicht betroffene Seite & & & & 0,322 \\
PPT Trochanter major & 0,385 & $\mathbf{0 , 0 2 1}$ & 0,269 & $\mathbf{0 , 0 4 4}$ \\
PPT M. gluteus medius & 0,420 & $\mathbf{0 , 0 2 1}$ & 0,079 \\
PPT M. vastus medialis & 0,420 & $\mathbf{0 , 0 2 1}$ & 0,355 & $\mathbf{0 , 0 3 0}$ \\
PPT M. vastus lateralis & 0,537 & $\mathbf{0 , 0 0 2}$ & 0,493 & $\mathbf{0 , 0 0 3}$ \\
PPT M. tibialis anterior & 0,399 & $\mathbf{0 , 0 2 9}$ & 0,329 & $\mathbf{0 , 0 4 0}$ \\
\hline
\end{tabular}

Tabelle 6: Signifikante Korrelationen des Body Mass Index mit Variablen der QST vor und mit Korrektur für den Faktor Geschlecht 


\section{Diskussion}

\subsection{Diskussion der Ergebnisse}

Insgesamt zeigen sich relevante Gruppenunterschiede in Messungen der QuantitativSensorischen Testung die als Hinweise auf prinzipielle Sensibilisierungen innerhalb der nozizeptiven Achse bei Menschen mit chronischer Hüftgelenksarthrose gedeutet werden können. Die Veränderungen konnten sowohl lokoregional als auch hüftfern (insbesondere für die PPT) nachgewiesen werden, als Hinweis auf eine zentrale Sensibilisierung.

Dieser Unterschied war unabhängig von bestehenden grundsätzlichen Unterschieden in der Schmerzempfindlichkeit von Männern und Frauen. Die Schmerzausdehnung in Form der Schmerzzeichnung korrelierte mit dem Ausmaß der funktionellen Beeinträchtigung und der Schmerzempfindlichkeit, im Einklang mit der Annahme einer zentralen Sensibilisierung als zusätzlichem Faktor des individuellen Schmerzerlebens. In den funktionellen und schmerzpsychologischen Fragebögen zeigte sich eine geringe psychologische und moderate funktionelle Beeinträchtigung der Patienten mit Hüftarthrose. Folglich ließ sich in dem in dieser Studie untersuchten Patientenkollektiv vor allem ein die (Alltags-)Funktion beeinträchtigendes Schmerzmuster nachweisen, ohne höhergradige oder fortgeschrittene Schmerzchronifizierung, aber bereits mit konsistenten psychophysiologischen Hinweisen einer zentralen Sensibilisierung, wie der Zusammenhang zwischen Schmerzsensibilität und Schmerzausdehnung.

\subsubsection{Gruppencharakteristika und Fragebögen}

Betrachtet man das Schmerzprofil der Probanden unserer Studie kann zusammenfassend festgehalten werden, dass zwar Patienten mit durchaus eingeschränkten Alltagsfunktionen, aber nur mäßig chronifizierten Schmerzen teilnahmen. So ist der Anteil der Probanden, die mindestens 30 Punkte im Oxford Hip Score erreichten, mit $43 \%$ durchaus hoch (Murray et al. 2007), während die oben beschriebene Verteilung der Probanden auf die Von-Korff-Grade lediglich einen geringen Schweregrad im Sinne einer chronischen Schmerzerkrankung anzeigt. Psychologische Faktoren waren ebenfalls wenig auffällig, als potentieller Hinweis auf eine relevante Chronifizierung. Zu dieser Konstellation passt, dass zwar für die physische, jedoch nicht für die psychische Skala des SF-36 auffällige Werte bezüglich krankheitsbedingt erniedrigter Lebensqualität erhoben wurden.

Außerdem zeigte sich bereits eine Korrelation zwischen niedrigerer Druckschmerzschwelle und höherer Funktionseinschränkung. Eine mögliche Erklärung für die geringe Chronifizierung ist sicherlich, dass unsere Studienteilnehmer mit durchschnittlich 7,2 ( \pm 8$)$ Jahren Beschwerden 
eher am Anfang ihrer Erkrankung stehen. Auch scheint an dieser Stelle eine konservative Therapie noch ausreichend effektiv oder nicht ausgereizt und daher der Grad der Chronifizierung womöglich nicht ausreichend, um eine erhöhte Erregbarkeit der Sinne durch beispielsweise Emotion oder Stress mit den vorliegenden Methoden zu messen. Auch Erlenwein (2017) konnte bei Patienten mit Koxarthrose unmittelbar vor Hüftgelenksersatz zwar einen hohen Schweregrad schmerzbedingter Funktionsbeeinträchtigung nach Von-Korff, aber nur eine geringe bis moderate Schmerzchronifizierung zeigen. In Studien konnte gezeigt werden, dass Übergewicht als Risikofaktor für verschiedene Formen der Arthrose gilt (Reyes et al. 2016). Weiterhin interessant ist, dass übergewichtige Patienten mit Arthrose mehr Schmerzen empfinden als normalgewichtige mit vergleichbaren strukturellen Schäden im Gelenk (Goulston et al. 2011). Vor diesem Hintergrund ist die Gewichtsreduktion eine elementare Maßnahme, um die biomechanische Belastung auf das Gelenk und damit den womöglich am ehesten direkt beeinflussbaren Risikofaktor zu minimieren (Bruyère et al. 2015). Innerhalb unserer Studie ergab sich ein mittlerer Wert von $25 \mathrm{~kg} / \mathrm{m}_{2}$ (SD 4) für den BMI der Gruppe mit Koxarthrose und 24 $\mathrm{kg} / \mathrm{m}_{2}$ (SD 4) für den der Kontrollgruppe. Beide Werte befinden sich im oberen Bereich des Normalgewichts mit Tendenz zur Präadipositas und waren weder zwischen den Gruppen $(p=0,30)$, noch den Geschlechtern innerhalb der beiden Gruppen $(p>0,05)$ signifikant verschieden. Allerdings konnten positive Korrelationen zwischen der Höhe des Body Mass Index und einigen Werten der Druckschmerzschwellen gemessen werden. Der Einfluss des Faktor Geschlecht auf diese Korrelationen war nur gering. Dies unterstreicht sicherlich, dass der BMI geschlechtsunabhängig ein relevanter Faktor in der Genese der Arthrose darstellt, allerdings in dieser Studie die Adipositas als potenzieller Einfluss eher zu vernachlässigen zu sein scheint.

Psychologische Komorbiditäten wie Depression, Angst und Stress können unter anderem mithilfe des gleichnamigen Fragebogens (DASS) gemessen werden, der im Kontext von chronischen Schmerzerkrankungen validiert wurde (Nilges und Essau 2015). Wie bereits oben erwähnt, fanden sich diesbezüglich in unserer Studie keine erhöhten Werte. Dies ist vergleichbar mit den Ergebnissen von Rice et al. 2016, die zwar für eine Kohorte mit chronischen Schmerzen (Definition: Schmerzdauer mindestens 3 Monate) erhöhte Werte im DASS messen konnten, jedoch nicht für die Kohorte mit rheumatoider Arthritis (RA). Auch die durchgeführte Testung auf erhöhte Werte in der Pain Catastrophizing Scale (PCS) ergab vornehmlich erhöhte Werte innerhalb der Kohorte mit chronischem Schmerz, während die Kohorte mit rheumatoider Arthritis nur mäßig erhöhte Werte erreichte (Rice et al. 2016). Am ehesten zu erklären scheint dieses Phänomen mit der höheren Dauer des Leidensdruckes bei den Patienten mit chronischen Schmerzen gegenüber der Kohorte mit isolierter rheumatoider Arthritis, die noch nicht chronifiziert zu sein schien. Muskuloskelettale Erkrankungen wie die RA oder auch die Arthrose 
wie in unserer Studie, scheinen besonders in frühen Stadien eher nicht mit gravierenden schmerzpsychologischen Komorbiditäten assoziiert zu sein. Im weiteren Verlauf von Schmerzerkrankungen kann es dann allerdings häufiger durch kognitive Prozesse, Schmerzerwartung und Aspekte des Fear Avoidance Modells zu chronifizierten Zuständen kommen (Pfingsten et al. 2001). Diese könnten dann mithilfe verschiedener Fragebögen gemessen werden.

Bezüglich der allgemeinen Lebensqualität, gemessen durch den SF-36 Health Survey, konnten, wie bereits erwähnt, erniedrigte Werte für die physische, nicht aber für die psychische Achse gemessen werden. Für zahlreiche schmerzhafte Erkrankungen konnte festgestellt werden, dass neben dem eigentlichen Schmerz besonders die Einschränkung der Lebensqualität und Alltagsaktivität gravierende zusätzliche Belastungen für die Patienten bedeuten können (Gerbershagen et al. 2008, Eriksen et al. 2009).

Dies passt zu den Erkenntnissen von Salaffi et al., die ebenfalls erniedrigte Werte für die gesundheitsbezogene Lebensqualität bei Gelenkarthrose messen konnten (Salaffi et al. 2005). Allerdings konnten hier Veränderungen im Gruppenvergleich zu Gesunden sowohl in der physischen als auch in der psychischen Achse beobachtet werden. Dies könnte im Vergleich zu unserer Studie eventuell auch an der deutlich größeren Teilnehmeranzahl und den mitunter schwerer betroffenen Teilnehmern liegen. 


\subsubsection{Diskussion der Gruppenunterschiede}

Die Messung von Druckschmerzempfindlichkeit über Muskeln und Sehnenansätzen kann nachweislich Informationen über erniedrigte Schwellen und damit über eine mögliche Überempfindlichkeit an verschiedenen Muskelpunkten bringen (Fischer 1987).

Die Ergebnisse der erniedrigten Druckschmerzschwellen im Gruppenvergleich unserer Studie legen eine Beteiligung zentraler Komponenten in der Schmerzgenese nahe. So waren tiefere Werte für Druckschmerzempfindlichkeit nicht nur hüftgelenksnah, sondern auch in gelenkfernen Regionen zu messen. In der Literatur finden sich auch für andere chronische Schmerzerkrankungen ähnliche Ergebnisse. Erniedrigte Schwellen für Druckschmerz im Gruppenvergleich konnten unter anderem auch für Patienten mit rheumatoider Arthritis (Dhondt et al. 1999), Spondylitis ankylosans (Gerecz-Simon et al. 1989), chronischem Rückenschmerz (O’Neill et al. 2007) und dem Fibromyalgie-Syndrom (Granges und Littlejohn 1993) nachgewiesen werden. Auch für Erkrankungen aus dem Formenkreis der Arthrose wurden vergleichbare Erkenntnisse gewonnen. So wurden neben gelenknahen Unterschieden für Druckschmerz bei Gonarthrose im Gruppenvergleich mit Gesunden auch signifikante Unterschiede im Bereich des Unterarms (Graven-Nielsen et al. 2012) sowie des Daumens und der Schulter (Lee et al. 2011) gemessen. Bezüglich Hüftarthrose konnten Kosek und Ordeberg bereits im Jahr 2000 niedrigere Schwellen für Druckschmerz auf der von Arthrose betroffenen Seite, nicht aber auf der gesunden kontralateralen Seite nachweisen (Kosek und Ordeberg 2000a).

Selbst bei insgesamt selteneren Arthroseformen wie der Rhizarthrose konnten geringere Druckschmerzschwellen für gelenknahe und -ferne Bereiche beschrieben werden (Chiarotto et al. 2013). Mit unseren Ergebnissen überein stimmen auch Erkenntnisse von O’Driscoll und Jayson aus dem Jahr 1974, die bei bekannter Koxarthrose auch erniedrigte Druckschmerzschwellen für eigentlich neutrale Körperregionen wie beispielsweise der Stirn fanden (O’Driscoll und Jayson 1974).

Die Zusammenschau dieser Ergebnisse, auch in Kombination mit den auffälligen Schmerzkarten und den immerhin $63 \%$ der Teilnehmer mit mindestens drei schmerzhaften Körperregionen im Widespread Pain Index, spricht für Anzeichen einer erhöhten generalisierten Schmerzempfindung. An dieser Stelle sollte aber bereits angemerkt werden, dass Frauen in den digitalen Schmerzkarten eine deutlich größere Schmerzausdehnung zeichneten als Männer. Dies wird in Kapitel 4.1.4 nochmals diskutiert werden.

Bezüglich der thermalen Schmerzschwellen fällt auf, dass nur zwei Werte signifikante Unterschiede im Gruppenvergleich zeigen (Tabelle 5). Dies steht im Gegensatz zu den Ergebnissen von Kosek und Ordeberg (2000a), die in der gleichen Studie beschrieben, dass 
Patienten mit Hüftgelenksarthrose sowohl auf der von Arthrose betroffenen Seite als auch kontralateral signifikant niedrigere Schwellen für verschiedene thermische Stimuli im Vergleich zu Gesunden aufweisen können (Kosek und Ordeberg 2000a). Interessanterweise normalisierten sich diese Gruppenunterschiede nach erfolgter Hüft-TEP, genauso wie die Unterschiede im Druckschmerz zwischen betroffener und nicht betroffener Seite. Auch dies steht in gewisser Weise in Widerspruch zu unseren Ergebnissen, die ein erhöhtes generalisiertes Schmerzempfinden nahelegen, während die oben genannte Studie lediglich eine unilaterale Veränderung der Schmerzempfindung zeigt. An dieser Stelle ist jedoch hinzuzufügen, dass die klare Begrenzung auf unilaterale Symptome bei Koxarthrose nicht immer klar möglich ist. Es konnte gezeigt werden, dass selbst radiologisch und klinisch völlig unauffällige Gelenke zum Zeitpunkt der Hüftgelenksersatz-Operation der kontralateralen Seite innerhalb von 10 Jahren ein Risiko von 36,5 \% haben, ebenfalls arthrotisch zu verändern (Ritter et al. 1996). Unklar bleibt zudem, ob und in welcher Reihenfolge sich die Sensibilität auf Druck, Kälte oder Hitze als Ausdruck einer zentralen Sensibilisierung überhaupt verändert.

\subsubsection{Prüfung der klinischen Kriterien für zentrale Sensibilisierung}

Wie bereits beschrieben, schlugen Nijs und Kollegen im Jahr 2014 eine Reihe von Kriterien vor, um Anzeichen einer zentralen Sensibilisierung bei chronischen Schmerzerkrankungen klinisch zu erkennen (Nijs et al. 2014).

Die Empfehlung der Autoren, zunächst Patienten mit klar neuropathischen Schmerzen auszuschließen, stellt sich als wesentlich schwieriger heraus als zunächst vermutet, da die Schmerzgenese anamnestisch und diagnostisch häufig nicht vollständig aufzuklären ist und die Symptome sich klinisch zumindest ähnlich darstellen können. Auf jeden Fall ist die klinische Einschätzung eines neuropathischen Schmerzsyndroms von einer Erhebung neuropathischer Schmerzcharakteristika mit einem Screening Fragebogen zu trennen (van Hecke et al. 2015). Diesbezüglich konnte zum Beispiel gezeigt werden, dass Patienten mit Gonarthrose mit höheren Werten in einer Variation des painDETECT-Fragebogens für neuropathischen Schmerz klinisch auch mehr Zeichen einer zentralen Sensibilisierung in der QST zeigen (Hochman et al. 2013). Bei Schmerzsyndromen wie dem Fibromyalgie-Syndrom (FMS) zeigen sich ähnliche Zusammenhänge (Amris et al. 2010). Weiterhin geben Studien Hinweise darauf, dass sich Qualitäten wie z.B. die Schmerzintensität, Schmerzdauer oder der Zeitpunkt des Auftretens von Schmerzen zwischen Patienten mit klar neuropathischen und muskuloskelettalen Schmerzen mit zentraler Sensibilisierung klinisch durchaus vergleichen lassen (Schäfer et al. 2017). In Anbetracht dieser Ergebnisse scheint eine Trennung der Probanden mit klar neuropathischen Schmerzen aufgrund eines Screeningfragebogens wie 
painDETECT kaum realistisch und wurde in unserer Analyse daher nicht durchgeführt. Aus klinischer Sicht war bei keinem unserer Probanden mit einem auffälligen painDETECT eine eindeutige und erklärende neurologische Diagnose bekannt.

\section{Kriterium 1 ("Disproportion of pain to the nature and extent of injury or pathology")}

Dies könnte am ehesten anhand eines Vergleiches von Ausprägung der Arthrose im Röntgenbild bzw. Kellgren and Lawrence-Klassifikation und Ausmaß der geäußerten Schmerzen geprüft werden. Wie bereits beschrieben, verzichteten wir jedoch bewusst auf die Sichtung weiterer radiologischer Bilder im Vorfeld der Studie, da die Diskrepanz zwischen röntgenologischen Auffälligkeiten und Klinik bekannterweise groß ist (Finan et al. 2013). Somit bleibt die Einschätzung ob der objektive Grad der biologischen Schädigung (Röntgenbild) mit dem Ausmaß der Schmerzen korreliert oder nicht, in diesem Fall schwierig zu prüfen. Eine weitere Möglichkeit der Prüfung wäre die klinische Einschätzung der Patienten, die aufgrund des relativ moderat betroffenen Patientenkollektivs allerdings nur wenig sinnvoll erscheint. Außerdem mangelt es schlicht an operationalisierten Kriterien einer klinischen Einschätzung.

\section{Kriterium 2 ("Diffuse pain distribution + byperalgesia / allodynia")}

Das zweite Kriterium kann formal für die Gruppe der Probanden mit Koxarthrose insgesamt als erfüllt bewertet werden. In unserer Studie zeigten sich sowohl in der QST als auch in den Fragebögen und den Schmerzkarten starke Hinweise auf eine generalisiert erhöhte Schmerzwahrnehmung und Schmerzausbreitung inklusive vor allem mechanischer Hyperalgesie bei Probanden mit Koxarthrose.

\section{Kriterium 3 ("Hypersensitwity of senses unrelated to the musculoskeletal system")}

In Zusammenschau der unauffälligen Werte für schmerzpsychologische Komorbiditäten in den entsprechenden Fragebögen und den QST Ergebnissen kann Kriterium drei für die untersuchte Stichprobe formal nicht erfüllt werden. Zwar ergaben sich in unserer Studie leichte Gruppenunterschiede bezüglich der Wahrnehmung von Umweltreizen wie Wärme oder Kälte, allerdings keine relevanten schmerzpsychologischen Komorbiditäten oder Chronifizierungsfaktoren, vor allem in Bezug auf Angst, Emotionalität und Wahrnehmung von Stress.

Die Autoren führen weitere Symptome auf, die abseits der drei Kriterien weitere, wenn auch unspezifische Hinweise auf das Vorliegen von zentraler Sensibilisierung geben können. Zu diesen zählen beispielsweise Schlafstörungen, Taubheit einer oder mehrerer Extremitäten, kognitive Defizite oder Gelenksteife ohne objektive Ursachen (Nijs et al. 2014). In unserer 
Studie nutzen wir die Subskala Symptom Severity Score (SSS) des Fibromyalgia Survey Fragebogens für die Erhebung einiger dieser Symptome. Allerdings erfüllten lediglich $14 \%$ der Teilnehmer die Kriterien. Der Großteil unserer Probanden wies weder Kopfschmerzen, depressive Verstimmungen oder viszerale Schmerzen, noch Tagesmüdigkeit, nicht-erholsamen Schlaf oder Gedächtnisprobleme auf. Eventuell wäre in Zukunft alternativ die Anwendung des weiter oben beschriebenen Central Sensitization Inventory zu diskutieren.

Zusammenfassend konnte lediglich ein Kriterium der Kollegen Nijs et al. in unserer Studie eindeutig erfüllt werden, wobei das erste Kriterium für die Diagnose Arthrose unzureichend operationalisiert ist. So fanden sich klare Hinweise auf eine generalisierte Verstärkung der Schmerzwahrnehmung bei unseren Probanden mit Koxarthrose in der Testung auf Druckschmerz, der Schmerzausdehnung und den Fragebögen für widespread pain, aber keine zusätzlichen schmerzpsychologischen Aspekte. Die beiden anderen Kriterien wurden nicht oder nur zum Teil erfüllt. Dies kann aber durchaus als psychophysiologisches Korrelat einer zentralen Sensibilisierung in einem insgesamt wenig chronifizierten Kollektiv erklärt werden. Die Daten weisen somit auf ein Kontinuum der zentralen Sensibilisierung hin mit interagierenden nozizeptiven und psychologischen Faktoren, die je nach Krankheitsbild unterschiedliche Bedeutung haben könnten.

Als möglicher Grund sei an dieser Stelle nochmals auf die Zusammensetzung unserer Probanden hingewiesen, die augenscheinlich erst am Beginn ihrer Erkrankung stehen. Vermutlich haben hier, wenn überhaupt, erst wenige neuroplastische Prozesse stattgefunden, die zu einer messbaren Chronifizierung führen können. Die Durchführung einer Studie mit im Alltag noch schwerer limitierten Probanden oder eine longitudinale Untersuchung, wie sie beispielsweise für den Rückenschmerz vorliegen (Tesarz et al. 2016) könnte hier weitere Erkenntnisse liefern. 


\subsubsection{Diskussion der Geschlechtsunterschiede}

Die signifikanten Geschlechtsunterschiede im QST und der Schmerzausdehnung in den Schmerzkarten haben laut durchgeführter two-way mixed-ANOVA in unserer Studie keinen Einfluss auf die Gruppenunterschiede bezüglich der gemessenen Druckschmerzempfindlichkeit. In der Literatur gibt es allerdings Hinweise darauf, dass der Faktor Geschlecht durchaus Einfluss auf Aspekte der zentralen Sensibilisierung haben kann (Sluka et al. 2012, Bartley et al. 2016). Insgesamt finden sich uneinheitliche epidemiologische Daten zur Verteilung von chronischen Schmerzen auf beide Geschlechter. Der Großteil der Ergebnisse legt allerdings nahe, dass Frauen generell häufiger als Männer betroffen sind und gleichzeitig auch häufiger an schmerzpsychologischen Komorbiditäten leiden (Fillingim et al. 2009). Auch ein potentieller Einfluss des Geschlechts auf widespread pain wird nicht einheitlich bewertet. So gibt es Studien, die keinen signifikanten Einfluss des Geschlechts finden konnten (Bergman et al. 2002, Gupta et al. 2007), während andere durchaus von einem relevanten Einfluss berichten (Bartley et al. 2016).

Für einen Einfluss des Geschlechts in unserer Studie spricht die Auswertung der Schmerzkarten, in denen Frauen eine signifikant höhere Schmerzausbreitung zeigten als Männer $(\mathrm{p}<0,007)$. Auch für andere chronische Schmerzerkrankungen wie chronischer Rücken- und Nackenschmerz zeichneten Frauen in digitalen Schmerzkarten eine höhere Gesamtfläche ihrer Schmerzen ein als Männer (Barbero et al. 2015).

Interessanterweise gab es in unserer Studie keine signifikanten Unterschiede in den weiteren Schmerzfragebögen für schmerzpsychologische Kofaktoren zwischen beiden Geschlechtern $(\mathrm{p}>0,05)$, sodass die Unterschiede in Schmerzempfindlichkeit und Schmerzausdehnung eher auf grundsätzliche Unterschiede im schmerzverarbeitenden System als auf die Relevanz psychosozialer Faktoren hinweisen.

In der Literatur zeigen Frauen generell niedrigere Schwellen für Druckschmerz (Riley et al. 1998), Wärme- und auch Kälteschmerz (Fillingim et al. 2009). Diese These lässt sich in unserer Studie sowohl für die Druckschmerzschwellen als auch für die thermischen Schwellen bestätigen. Da sich in unserer Studie allerdings nur geringe Gruppenunterschiede zwischen Gesunden und Menschen mit Hüftarthrose für thermischen Schmerz zeigten, sahen wir davon ab, eine two-way mixed ANOVA mit diesen Daten durchzuführen, da wir die Relevanz als begrenzt einstufen. Denn gerade für thermische Messgrößen konnte nachgewiesen werden, dass diese durch eine Reihe von unterschiedlichen Faktoren wie beispielsweise Hauttemperatur, Alter und Body Mass Index (de Kruijf et al. 2015) leicht beeinflussbar sind. In diesem Fall wäre gegebenenfalls noch zu prüfen, ob in unserer Studie Kofaktoren Einfluss auf die Temperaturschwellen hatten. 
Auch ist zu diskutieren, ob Patienten mit höhergradiger Koxarthrose signifikant auffällige psychologische Fragebögen zeigen würden, wie zuvor bereits für Patienten unmittelbar präoperativ gezeigt (Erlenwein et al. 2017) oder auch die gemessenen Geschlechtsunterschiede erneut bestätigen würden.

Insgesamt können in dieser Studie bei beiden Geschlechtern Zeichen einer zentralen Sensibilisierung nachgewiesen werden, hier gemessen durch die Druckschmerzschwellenwerte und die Schmerzausdehnung, wobei Frauen für beide Parameter grundsätzlich die empfindlicheren Werte angeben als Männer. Auch die Korrelationen zwischen Druckschmerzschwellen und dem Oxford Hip Score als Messinstrument einer funktionellen Alltagsbeeinträchtigung durch das Hüftgelenk sind durch den Faktor Geschlecht in unserer Studie messbar abgeschwächt (Kapitel 3.6.4).

\subsection{Einschränkungen und Stärken der Arbeit}

Man könnte einwenden, dass der Verzicht auf eine standardisierte Befundung von Röntgenbildern eine relevante Einschränkung bezüglich der Rekrutierung darstellt, da wir uns auf die bereits gestellte Diagnose der Koxarthrose verließen. Allerdings kann man dem entgegenstellen, dass wie oben beschrieben, häufig eine Diskrepanz zwischen radiologischem Score und Klinik besteht (Finan et al. 2013, Kim et al. 2015), auch wenn ältere Studien gegenteilige Ergebnisse lieferten (Duncan et al. 2007, Neogi et al. 2009). Für unsere Stichprobe scheint stattdessen eine funktionelle Klassifikation wie der Oxford Hip Score besser geeignet, um den Schweregrad der Erkrankung abschätzen zu können. Bezüglich des Schweregrades der Arthrose bei unseren Probanden muss man konstatieren, dass vornehmlich Patienten teilnahmen, die zwar alltagseingeschränkt und schmerzgeplagt waren, für den Großteil allerdings noch keine Indikation für einen operativen Gelenkersatz bestand. Zu berichten ist auch, dass zwei Probanden formal die Punktzahl im painDETECT erreichten, die eine neuropathische Beteiligung in der Genese der Schmerzen nahelegt. Außerdem kommen drei Probanden aufgrund des Ergebnisses des FMS Fragebogens differentialdiagnostisch für ein Fibromyalgie-Syndrom in Frage. Allerdings gab es klinisch weder in der Anamnese noch in der körperlichen Untersuchung im Vorfeld Anzeichen für diese Komorbiditäten, sodass wir uns entschieden, die betroffenen Probanden nicht auszuschließen. Vor dem Hintergrund, dass zwischen einer neuropathischen Genese und einer Mitbeteiligung von Aspekten der zentralen Sensibilisierung klinisch nicht immer klar unterschieden werden kann (Schäfer et al. 2017), erscheint dies legitim. 
Eine weitere Einschränkung der Arbeit ist die fehlende Vergleichbarkeit der Fragebögen zwischen Hüftarthrose- und Kontrollgruppe, da versäumt wurde, den Kontrollprobanden ebenfalls Exemplare der geeigneten Schmerzfragebögen, insbesondere den SF-36, auszuhändigen. Daher mussten Ergebnisse aus anderen Arbeiten zum Vergleich herangezogen werden. Die Verwendung etablierter Methoden wie der Quantitativ-Sensorischen Testung zur Detektion etwaiger Hinweise auf eine zentrale Beteiligung in der Schmerzgenese unserer Probanden zählt sicherlich zu einer der Stärken. Nach dem Protokoll von Rolke et al. 2006 ist es prinzipiell möglich alle physiologisch beteiligten Bahnen in der nozizeptiven Achse zu testen und damit Hinweise auf gestörte Prozesse in den spinothalamischen Achsen oder Hinterstrangbahnen zu erhalten (Rolke et al. 2006b). Allerdings ist auch anzumerken, dass das System nicht explizit für die Detektion von zentraler Sensibilisierung validiert wurde. Weiterhin trägt diese Studie sicherlich dazu bei, dass die komplexe Genese chronischer Schmerzerkrankungen, wie der Hüftarthrose und deren Therapie, Gegenstand der Forschung bleibt. Die Studie verdeutlicht auch, dass Kriterien wie die von Nijs et al. 2014 vor dem Übergang in die klinische Anwendung kritisch geprüft und validiert werden müssen. 


\subsection{Ausblick}

Nicht-tumorassoziierte chronische Schmerzen stellen in unserer Gesellschaft eine relevante Problematik dar. Sozioökonomisch betrachtet steigen die Ausgaben für kurative und rehabilitative Maßnahmen bei chronischen Erkrankungen kontinuierlich an und sorgen damit für hohe direkte Kosten für das Gesundheitssystem. Zwischen 13-51 \% der Patienten mit chronischen Schmerzen erhalten laut Wolff et al. zudem keine adäquate Therapie (Wolff et al. 2011).

Nach Erkrankungen aus dem kardiovaskulären Formenkreis sind vor allem muskuloskelettale Erkrankungen am ehesten der Grund für hohe gesundheitswirtschaftliche Ausgaben in Europa (Breivik et al. 2013). Global gesehen stellt die Arthrose eins der wichtigsten Krankheitsbilder dar, das zu starken funktionellen Einschränkungen im Alltag führt und damit auch zu indirekten Kosten beiträgt. Es wird prognostiziert, dass die Erkrankung in unserer weiter alternden Gesellschaft zu einer Herausforderung für das Gesundheitssystem werden könnte (Cross et al. 2014). Derzeit besteht die einzig kurative Therapiemöglichkeit im chirurgischen Hüftgelenksersatz. Weltweit wird diese Operation immer häufiger durchgeführt (Wengler et al. 2014). Deutschland ist nach der Schweiz sogar das Land mit der höchsten Frequenz an Hüftgelenksersatzoperationen (OECD 2013). Mit über 212000 durchgeführten Eingriffen im Jahr 2012 zählte die Operation zu den sieben häufigsten operativen Eingriffen in Deutschland überhaupt (Jaschinski et al. 2014).

Dies wird zunehmend kritisch gesehen, da unter anderem gezeigt werden konnte, dass insbesondere ältere Patienten die endoprothetisch versorgt wurden, mitunter prolongierte Rehabilitationszeiten benötigen und trotzdem häufig nicht wieder vollständig in den aktiven Alltag zurückkehren (Horstmann et al. 2001) oder die Schmerzen persistieren können (Lundblad et al. 2008). Auch die Gefahr einer postoperativen Schmerzchronifizierung bei präoperativ bestehender schmerpsychologischer Veranlagung sorgt für eine zunehmend differenzierte Betrachtung der operativen Intervention. Allerdings ist anzumerken, dass chronischer Schmerz nach wie vor uneinheitlich definiert ist und gleichzeitig die Unterscheidung zwischen schon präoperativ vorhandenem und erst durch die Operation entstandenem Schmerz regelmäßig Schwierigkeiten bereitet (Erlenwein et al. 2017).

Dem gegenüber stehen Studien, die zeigen konnten, dass sich erhöhte Werte in patientenorientierten Fragebögen wie dem Oxford Hip Score nach erfolgtem Hüftgelenksersatz normalisierten (Wylde et al. 2009) und Patienten postoperativ eine deutliche Schmerzerleichterung verspüren. Gleichzeitig konnte belegt werden, dass sich bei Patienten mit Arthrose des Kniegelenks nach erfolgtem operativen Gelenkersatz nicht nur eine Besserung der Schmerzen und Steigerung der Lebensqualität (Kirwan et al. 1994), sondern auch eine 
Normalisierung der zuvor gemessenen Hyperalgesie an gelenkfernen Regionen einstellte (Graven-Nielsen et al. 2012).

Bis ein Gelenkersatz unumgänglich ist, wird die Koxarthrose häufig lange konservativ therapiert und der Fokus auf eine Maximierung der Lebensqualität, Funktionserhalt und Schmerzkontrolle gelegt. Dies kann unter anderem durch Beratungen zu Lebensstil und Gewichtsreduktion, konsequente Bewegung in Alltag und Beruf, unterstützende physiotherapeutische Übungen zur Stärkung der Muskulatur sowie eine medikamentöse Analgesie gelingen. Hierfür wurde lange Zeit der Einsatz von nicht-steroidalen Antiphlogistika empfohlen. Allerdings konnte gezeigt werden, dass neben vielen unerwünschten Arzneimittelwirkungen bei Langzeiteinnahme häufig auch nur unzureichende Schmerzerleichterungen resultieren (Malfait und Schnitzer 2013). Immer wieder wurde daher auch die Hinzunahme von zentral wirksamen Analgetika wie Antikonvulsiva und trizyklischen Antidepressiva sowie selektive Serotonin-WiederaufnahmeHemmer (SSRI) als potente Koanalgetika bei Hinweisen auf zentrale Komponenten in der Schmerzgenese diskutiert (Chappell et al. 2009). Im Tiermodell konnte gezeigt werden, dass im Vergleich zu herkömmlichen Analgetika, besonders zentral wirksame Koanalgetika wie z.B. Gabapentin eine relevante Schmerzreduktion bei Arthroseschmerz bedingten (Vonsy et al. 2009). Studien ergaben weiterhin, dass konservative Therapieregime bei chronischen Schmerzerkrankungen durchaus das Potenzial haben, stattgehabte neuronale Umbauvorgänge zu reduzieren (Seminowicz et al. 2013, Shpaner et al. 2014). Ferner ist es möglich durch intensivierte physiotherapeutische Maßnahmen im Kontext zentraler Sensibilisierung endogene inhibitorische GABA-erge Systeme zu aktivieren, die die Aktivität von WDR-Neuronen drosseln können und so frühzeitig einerseits Chronifizierungen aufhalten, aber auch Menschen mit bereits chronifizierte Schmerzleiden in die Aktivität zurückbringen können (George et al. 2006, O’Leary et al. 2016, Falla und Hodges 2017).

Dennoch aggravieren muskuloskelettale Schmerzen im Verlauf häufig (Brabant und Stichtenoth 2005). Solche Schmerzexazerbationen der Arthrose mit Zeichen nur geringer zentraler Komponente scheinen mithilfe von lokalen Maßnahmen wie Injektionen von Steroiden oder Hyaluronsäure, oder topisch mit beispielsweise Capsaicin behandelbar. Im Falle einer Beteiligung zentraler Komponenten wurde allerdings zuletzt auch ein multimodaler Therapieansatz nach dem biopsychosozialen Modell diskutiert. (Lluch Girbes et al. 2013). Dieser könnte beispielsweise aus Patientenedukation, Ansätzen kognitiver Verhaltenstherapie (Smith et al. 2015) und physiotherapeutischer Behandlung (Clauw und Hassett 2017) bestehen. Für solche multimodalen schmerztherapeutischen Interventionen im Kontext chronischmuskuloskelettaler Erkrankungen konnte nicht nur eine erheblich verbesserte Lebensqualität, sondern ebenfalls signifikant reduzierte Werte für Angst, Depression und weitere 
schmerzpsychologische Komorbiditäten nachgewiesen werden (Borys et al. 2015). Besonders für die neurowissenschaftliche Edukation mit Ansätzen kognitiver Verhaltenstherapie konnten bislang gute Ergebnisse bescheinigt werden (Louw et al. 2011). Manche Autoren gehen noch einen Schritt weiter und diskutieren, ob Patienten mit Arthrose nicht auch im Rahmen personalisierter Konzepte ganzheitlicher therapiert werden könnten (Karsdal et al. 2014). Hier dient zum Beispiel die personalisierte Therapie der rheumatoiden Arthritis als Vorbild, bei der unterschiedliche klinische Phänotypen existieren können, die anhand ihrer individuellen Facetten therapiert werden (Karsdal et al. 2014). Insgesamt geht es also auch darum, den Fokus weg von eher eindimensionalen Schmerzkonzepten zu nehmen, nach denen bei Entfernung der (vermuteten) peripheren Schmerzquelle das negative Schmerzerleben der Patienten zwangsläufig sistieren müsste. Es sollte vielmehr darum gehen, chronische Schmerzen als mehrdimensionale Erkrankung zu verstehen, um dem Patienten die bestmögliche Linderung zu verschaffen sowie einen maximalen Funktionserhalt im Alltag mit Integration angemessener konservativer und operativer Maßnahmen.

Stellt man zusammenfassend die Vorteile der Hüftgelenksoperation den Vorteilen der breiten konservativen Therapiemöglichkeiten gegenüber, verbleibt derzeit dennoch nicht die Frage, ob eine Operation langfristig generell sinnvoll ist. Vielmehr stellt sich die Frage nach einem optimalen Zeitpunkt und einer geeigneten Patientenauswahl für eine solche Intervention (Altman et al. 2005). Denn solange die Arthrose zu den per se unheilbaren Erkrankungen zählt, bleibt für den Patienten am langen Ende einer konservativen Therapie häufig nur der operative Gelenkersatz mit überwiegend positiven Ergebnissen.

Zukünftig könnte sich der Umgang mit Patienten mit eindeutig zentralen Komponenten in der Schmerzentstehung durch eine Beteiligung verschiedener Fachdisziplinen verändern. So scheint eine ganzheitlich-maßgeschneiderte schmerztherapeutische Behandlung, bestehend aus pharmakologischen, psychologischen und physiotherapeutischen Aspekten, langfristig bei geeigneten Patienten durchaus realistisch, bedarf aber auch der Validierung in interventionellen Studien. An dieser Stelle scheinen gerade Patienten mit deutlichen Schmerzen, aber nur gering ausgeprägten degenerativen Befunden eine Sonderstellung einnehmen.

Nur durch weitere Erkenntnisse wird es möglich sein, die komplexen Zusammenhänge aus peripheren und zentralen Faktoren der Schmerzentstehung bei Arthrose besser zu verstehen und entsprechende Lösungen zu finden, unter Umständen auch mithilfe personalisierter Konzepte. 


\section{$5 \quad$ Zusammenfassung}

Die Hüftgelenksarthrose (Koxarthrose) ist eine der häufigsten degenerativen Erkrankungen weltweit und stellt im Kontext der zunehmenden Alterung der Gesellschaft eine unveränderte medizinische Herausforderung dar. Während für lange Zeit ein rein mechanisches Schmerzverständnis für Koxarthrose etabliert war, mit dem Fokus auf nozizeptiven und überwiegend peripheren Mechanismen scheint mittlerweile eine Beteiligung einer gestörten Schmerzverarbeitung (noziplastischer Schmerz) im Sinne einer zentralen Sensibilisierung plausibel. Vor dem Hintergrund zahlreicher unterschiedlicher Theorien zur Pathophysiologie der zentralen Sensibilisierung stellte sich die Frage der klinischen Relevanz und Nachweisbarkeit bei Patienten mit Koxarthrose. Ziel der durchgeführten Untersuchung war daher, Zeichen einer zentralen Sensibilisierung in einer Gruppe Patienten $(n=30)$ im Gruppenvergleich mit schmerzfreien Menschen ( $\mathrm{n}=18)$ zu messen. Die Patienten hatten eine nach ICD-10 diagnostizierte einseitig betonte Koxarthrose ohne aktuelle Indikation für einen Gelenkersatz. Sie füllten digitale Schmerzkarten, ein Fragebogenset mit Fokus auf Schmerzausdehnung und Charakteristik sowie schmerzpsychologische Faktoren aus und erhielten eine QuantitativSensorische Testung (QST) in hüftgelenksnahen und distalen Messorten. A priori waren für die Gruppe mit Koxarthrose niedrigere Schmerzschwellen für Druck- und Hitzereize und erhöhte Schmerzschwellen für Kältereize in gelenknahen und distalen Messpunkten zu erwarten sowie Hinweise für das Vorliegen möglicher Chronifizierungsfaktoren.

Die Auswertung der Fragebögen ergab das Vorliegen von funktionell im Alltag relevant eingeschränkten Probanden ohne nachweisbare Aspekte einer hochgradigen psychologischen Schmerzchronifizierung. Weiterhin zeigten sich signifikant niedrigere Druckschmerzschwellen im Gruppenvergleich sowohl über hüftgelenksnahen (Trochanter major $(\mathrm{p}<0,0001)$ und M. gluteus medius $(\mathrm{p}<0,001))$ als auch an peripheren Messorten $($ Thenar $(\mathrm{p}<0,0001)$ und $M$. tibialis anterior $(\mathrm{p}<0,002))$. Auch kontralateral zeigten sich im Gruppenvergleich sowohl periartikulär als auch hüftgelenksfern signifikant niedrigere Schwellen für Druckschmerz $(p<0,05)$. Dieser Gruppenvergleich wurde nicht durch Drittvariablen wie BMI, Alter oder Geschlecht beeinflusst, obwohl sich Frauen gegenüber Männern ubiquitär signifikant empfindlicher für Druckschmerz zeigten. Ein signifikanter Zusammenhang zwischen erhöhten Werten im Oxford Hip Score (=schlechtere Funktion) und niedrigeren Druckschmerzschwellen konnte sowohl über hüftgelenksassoziierten als auch über peripheren Messorten festgestellt werden. Signifikante Korrelationen zwischen der gemessenen Gesamtfläche der Schmerzausdehnung (digitale Schmerzkarten) mit schmerzpsychologischen Fragebögen und einigen Variablen der QST wurden festgestellt, ebenso wie Korrelationen des BMI mit verschiedenen Druckschmerzschwellen. 
Die signifikanten Gruppenunterschiede in Messungen der Quantitativ-Sensorischen Testung (hierbei insbesondere der Druckschmerzempfindlichkeit) können als Hinweise auf eine Sensibilisierung innerhalb der nozizeptiven Achse bei Menschen mit funktionell limitierender Hüftgelenksarthrose gedeutet werden, oder auch als noziplastische Veränderungen. Da die Veränderungen sowohl lokoregional als auch hüftfern nachgewiesen werden konnten, kann diese Sensibilisierung als zentral mit generalisiert erniedrigter Schmerzschwelle erklärt werden. Die Ergebnisse der Korrelation unterstützen diesen Zusammenhang. Diese Erkenntnisse decken sich mit Ergebnissen für andere chronische Schmerzerkrankungen und Formen der Arthrose und stellen eine wichtige messbare Möglichkeit zum Nachweis von Aspekten der zentralen Sensibilisierung dar.

Bisher ist insgesamt noch wenig darüber bekannt, über welche Mechanismen diese Sensibilisierung ausgelöst wird und welche Bedeutung sie für die Chronifizierung muskuloskelettaler Schmerzen hat. Es ist anzunehmen, das auch genetische Prädispositionen, Umweltfaktoren oder Persönlichkeitsanteile das Risiko einer zentralen Sensibilisierung im Kontext der Arthrose erhöhen, triggern oder aufrechterhalten können. Aus diesem Grund ist weitere Forschung im Kontext degenerativ-muskuloskelettaler Erkrankungen mit chronischen Schmerzen unabdingbar, auch vor dem Hintergrund der steigenden sozioökonomischen Bedeutung. 


\section{Anhang}

\subsection{Ergänzende Tabellen}

\begin{tabular}{lccccc}
\hline & \multicolumn{7}{c}{ Nicht betroffene Seite } & Betroffene Seite & \\
\hline & Mittelwert & SD & Mittelwert & SD & $\mathbf{p}^{*}$ \\
& Thermale Schwellenwerte $\left({ }^{\circ} \boldsymbol{C}\right)$ & & & \\
CPT Thenar & 29,9 & 1,4 & 30,0 & 1,1 & $\mathbf{0 , 9 3 9}$ \\
WDT Thenar & 34,0 & 0,8 & 33,9 & 0,7 & $\mathbf{0 , 3 8 6}$ \\
CPT Thenar & 20,8 & 9,8 & 15,3 & 7,8 & $\mathbf{0 , 0 0 6}$ \\
HPT Thenar & 41,0 & 4,3 & 41,9 & 4,1 & $\mathbf{0 , 0 1 0}$ \\
CDT Trochanter major & 28,4 & 1,9 & 28,6 & 1,7 & $\mathbf{0 , 5 0 3}$ \\
WDT Trochanter major & 37,2 & 3,9 & 37,4 & 3,8 & $\mathbf{0 , 7 7 9}$ \\
CPT Trochanter major & 11,7 & 10,7 & 16,2 & 10,0 & $\mathbf{0 , 0 0 1}$ \\
HPT Trochanter major & 45,3 & 3,9 & 45,2 & 3,6 & $\mathbf{0 , 8 4 6}$ \\
& Druckschmerzschwellen & $(\mathbf{k P a})$ & & & \\
PPT Trochanter major & 419 & 146 & 400 & 149 & $\mathbf{0 , 1 5 0}$ \\
PPT M. gluteus medius & 421 & 143 & 385 & 138 & $\mathbf{0 , 0 0 9}$ \\
PPT M. vastus medialis & 390 & 132 & 354 & 119 & $\mathbf{0 , 0 3 0}$ \\
PPT M. vastus lateralis & 363 & 162 & 360 & 149 & $\mathbf{0 , 8 6 9}$ \\
PPT M. tibialis anterior & 408 & 141 & 395 & 131 & $\mathbf{0 , 3 1 3}$ \\
PPT Thenar & 362 & 106 & 356 & 110 & $\mathbf{0 , 5 3 8}$ \\
\hline
\end{tabular}

Tabelle 7: QST-Ergebnisse für betroffene und nicht betroffene Seite innerhalb der Gruppe mit Hüftarthrose

*Unterschied betroffene und nicht betroffene Seite, T-Test: PPT, Mann-Whitney U Test: Thermale Schwellenwerte 


\begin{tabular}{|c|c|c|c|c|c|c|c|c|c|c|c|}
\hline & \multicolumn{5}{|c|}{ Gruppe mit Koxarthrose } & \multicolumn{6}{|c|}{ Kontrollgruppe } \\
\hline Nicht betroffene Seite & $\mathrm{n}$ & Min. & Max. & MW & SD & $\mathrm{n}$ & Min. & Max. & MW & SD & $\mathrm{p}^{*}$ \\
\hline \multicolumn{12}{|c|}{ Thermale Schwellenwerte $\left({ }^{\circ} \mathrm{C}\right)$} \\
\hline CDT Thenar & 30 & 23,6 & 31,3 & 29,9 & 1,4 & 18 & 27,3 & 31,4 & 29,7 & 1,2 & 0,296 \\
\hline WDT Thenar & 30 & 33,0 & 36,6 & 34,0 & 0,8 & 18 & 32,7 & 36,6 & 33,9 & 1,1 & 0,326 \\
\hline CPT Thenar & 30 & 1,0 & 31,3 & 20,8 & 9,8 & 18 & 0,0 & 22,6 & 13,4 & 7,7 & 0,018 \\
\hline HPT Thenar & 30 & 34,2 & 49,3 & 41,0 & 4,3 & 18 & 36,0 & 50,5 & 42,1 & 4,7 & 0,349 \\
\hline CDT Trochanter major & 30 & 21,6 & 31,1 & 28,4 & 1,9 & 18 & 26,1 & 30,8 & 29,1 & 1,4 & 0,282 \\
\hline WDT Trochanter major & 30 & 33,8 & 48,1 & 37,2 & 3,9 & 18 & 33,4 & 37,9 & 35,5 & 1,4 & 0,287 \\
\hline CPT Trochanter major & 30 & 0,0 & 26,2 & 11,7 & 10,7 & 18 & 0,0 & 25,6 & 13,2 & 10,3 & 0,613 \\
\hline HPT Trochanter major & 30 & 36,7 & 50,5 & 45,3 & 3,9 & 18 & 36,6 & 49,2 & 44,1 & 3,8 & 0,250 \\
\hline \multicolumn{12}{|c|}{ Druckschmerzschwellen (kPa) } \\
\hline PPT Trochanter major & 30 & 199 & 777 & 419 & 146 & 18 & 469 & 883 & 600 & 122 & 0,000 \\
\hline PPT M. gluteus medius & 30 & 205 & 701 & 421 & 143 & 18 & 386 & 846 & 557 & 158 & 0,006 \\
\hline PРT M. vastus medialis & 30 & 192 & 654 & 390 & 132 & 18 & 278 & 821 & 427 & 141 & 0,360 \\
\hline PPT M. vastus lateralis & 30 & 124 & 736 & 363 & 162 & 18 & 216 & 639 & 378 & 103 & 0,443 \\
\hline PPT M. tibialis anterior & 30 & 193 & 738 & 408 & 141 & 18 & 334 & 799 & 547 & 139 & 0,001 \\
\hline PPT Thenar & 30 & 210 & 583 & 362 & 106 & 18 & 361 & 671 & 494 & 89 & 0,000 \\
\hline Betroffene Seite & $\mathrm{n}$ & Min. & Max. & MW & $\mathrm{SD}$ & $\mathrm{n}$ & Min. & Max. & MW & SD & $\mathrm{p}$ \\
\hline \multicolumn{12}{|c|}{ Thermale Schwellenwerte $\left({ }^{\circ} \mathrm{C}\right)$} \\
\hline CDT Thenar & 30 & 25,2 & 31,3 & 30,0 & 1,1 & 18 & 27,7 & 31,2 & 30,0 & 0,9 & 0,915 \\
\hline WDT Thenar & 30 & 32,8 & 35,2 & 33,9 & 0,7 & 18 & 33,1 & 36,4 & 34,0 & 0,9 & 0,932 \\
\hline CPT Thenar & 30 & 0,0 & 28,5 & 15,3 & 7,8 & 18 & 0,0 & 26,6 & 14,1 & 7,8 & 0,709 \\
\hline HPT Thenar & 30 & 34,3 & 48,5 & 41,9 & 4,1 & 18 & 34,6 & 47,2 & 41,9 & 3,9 & 0,898 \\
\hline CDT Trochanter major & 30 & 24,4 & 30,8 & 28,6 & 1,7 & 18 & 25,2 & 31,0 & 28,7 & 1,8 & 0,815 \\
\hline WDT Trochanter major & 30 & 33,6 & 47,7 & 37,4 & 3,8 & 18 & 33,4 & 37,4 & 35,1 & 1,2 & 0,021 \\
\hline CPT Trochanter major & 30 & 0,0 & 26,8 & 16,2 & 10,0 & 18 & 0,0 & 25,1 & 14,4 & 10,3 & 0,291 \\
\hline HPT Trochanter major & 30 & 35,0 & 50,0 & 45,2 & 3,6 & 18 & 36,3 & 49,5 & 44,5 & 3,7 & 0,450 \\
\hline \multicolumn{12}{|c|}{ Druckschmerzschwellen (kPa) } \\
\hline PPT Trochanter major & 30 & 177 & 761 & 400 & 149 & 18 & 374 & 885 & 591 & 140 & 0,000 \\
\hline PPT M. gluteus medius & 30 & 179 & 675 & 385 & 138 & 18 & 346 & 817 & 544 & 153 & 0,001 \\
\hline PPT M. vastus medialis & 30 & 178 & 627 & 354 & 119 & 18 & 232 & 698 & 424 & 121 & 0,062 \\
\hline PPT M. vastus lateralis & 30 & 163 & 744 & 360 & 149 & 18 & 232 & 675 & 388 & 115 & 0,241 \\
\hline PPT M. tibialis anterior & 30 & 121 & 640 & 395 & 131 & 18 & 405 & 816 & 547 & 140 & 0,002 \\
\hline PPT Thenar & 30 & 170 & 592 & 356 & 110 & 18 & 289 & 684 & 482 & 101 & 0,000 \\
\hline
\end{tabular}

Tabelle 8: Ergebnisse der Quantitativ-Sensorischen Testung in der Übersicht, * Gruppenvergleich T-Test: PPT, Mann-Whitney U Test: Thermale Schwellenwerte 


\begin{tabular}{|c|c|c|c|c|c|c|c|c|c|c|c|}
\hline & \multicolumn{5}{|c|}{ Frauen mit Koxarthrose } & \multicolumn{6}{|c|}{ Männer mit Koxarthrose } \\
\hline Nicht betroffene Seite & $\mathrm{n}$ & Min. & Max. & MW & $\mathrm{SD}$ & $\mathrm{n}$ & Min. & Max. & MW & $\mathrm{SD}$ & $\mathrm{p}$ \\
\hline \multicolumn{12}{|c|}{ Thermale Schwellenwerte $\left({ }^{\circ} \mathrm{C}\right)$} \\
\hline CDT Thenar & 15 & 28,4 & 31,3 & 30,3 & 0,71 & 15 & 23,6 & 31,0 & 29,5 & 1,81 & 0,161 \\
\hline WDT Thenar & 15 & 33,0 & 34,0 & 33,5 & 0,34 & 15 & 33,3 & 36,6 & 34,4 & 0,90 & 0,004 \\
\hline CPT Thenar & 15 & 1,0 & 31,3 & 24,0 & 9,29 & 15 & 1,0 & 30,8 & 17,7 & 9,65 & 0,050 \\
\hline HPT Thenar & 15 & 34,3 & 46,2 & 39,0 & 3,73 & 15 & 34,2 & 49,3 & 42,9 & 4,00 & 0,010 \\
\hline CDT Trochanter major & 15 & 24,8 & 31,1 & 28,6 & 1,78 & 15 & 21,6 & 30,6 & 28,2 & 2,20 & 0,744 \\
\hline WDT Trochanter major & 15 & 33,8 & 48,1 & 37,3 & 4,62 & 15 & 34,0 & 46,7 & 37,1 & 3,24 & 0,461 \\
\hline CPT Trochanter major & 15 & 0,0 & 25,0 & 12,9 & 10,06 & 15 & 0,0 & 26,2 & 10,4 & 11,46 & 0,539 \\
\hline HPT Trochanter major & 15 & 36,7 & 49,9 & 44,7 & 4,13 & 15 & 38,3 & 50,5 & 45,8 & 3,80 & 0,367 \\
\hline \multicolumn{12}{|c|}{ Druckschmerzschwellen (kPa) } \\
\hline PPT Trochanter major & 15 & 199 & 714 & 364 & 137,17 & 15 & 259 & 777 & 473 & 138,69 & 0,023 \\
\hline PРT M. gluteus medius & 15 & 205 & 662 & 345 & 118,40 & 15 & 313 & 701 & 498 & 125,32 & 0,002 \\
\hline PРT M. vastus medialis & 15 & 192 & 630 & 334 & 117,29 & 15 & 239 & 654 & 446 & 126,27 & 0,013 \\
\hline PPT M. vastus lateralis & 15 & 124 & 543 & 272 & 110,22 & 15 & 262 & 736 & 455 & 156,39 & 0,001 \\
\hline PPT M. tibialis anterior & 15 & 193 & 580 & 346 & 103,55 & 15 & 291 & 738 & 470 & 150,22 & 0,045 \\
\hline PPT Thenar & 15 & 210 & 521 & 321 & 86,77 & 15 & 221 & 583 & 402 & 111,98 & 0,033 \\
\hline Betroffene Seite & $\mathrm{n}$ & Min. & Max. & MW & SD & $\mathrm{n}$ & Min. & Max. & MW & SD & $\mathrm{p}$ \\
\hline \multicolumn{12}{|c|}{ Thermale Schwellenwerte $\left({ }^{\circ} \mathrm{C}\right)$} \\
\hline CDT Thenar & 15 & 29,2 & 31,3 & 30,4 & 0,59 & 15 & 25,2 & 30,8 & 29,5 & 1,38 & 0,026 \\
\hline WDT Thenar & 15 & 32,8 & 34,8 & 33,6 & 0,55 & 15 & 33,2 & 35,2 & 34,2 & 0,64 & 0,015 \\
\hline CPT Thenar & 15 & 0,0 & 28,5 & 16,6 & 9,09 & 15 & 4,5 & 26,2 & 14,0 & 6,32 & 0,325 \\
\hline HPT Thenar & 15 & 34,3 & 47,1 & 41,0 & 4,61 & 15 & 38,1 & 48,5 & 42,9 & 3,33 & 0,389 \\
\hline CDT Trochanter major & 15 & 25,0 & 30,8 & 29,0 & 1,76 & 15 & 24,4 & 30,5 & 28,3 & 1,68 & 0,148 \\
\hline WDT Trochanter major & 15 & 33,6 & 47,7 & 37,0 & 4,37 & 15 & 34,2 & 47,4 & 37,9 & 3,29 & 0,116 \\
\hline CPT Trochanter major & 15 & 0,0 & 26,8 & 19,9 & 8,06 & 15 & 0,0 & 25,6 & 12,5 & 10,69 & 0,033 \\
\hline HPT Trochanter major & 15 & 35,0 & 50,0 & 43,5 & 4,17 & 15 & 42,6 & 49,9 & 46,9 & 1,99 & 0,016 \\
\hline \multicolumn{12}{|c|}{ Druckschmerzschwellen (kPa) } \\
\hline PPT Trochanter major & 15 & 177 & 761 & 347 & 160,98 & 15 & 295 & 693 & 453 & 119,57 & 0,021 \\
\hline PPT M. gluteus medius & 15 & 179 & 668 & 322 & 144,45 & 15 & 306 & 675 & 447 & 99,84 & 0,002 \\
\hline PРТ M. vastus medialis & 15 & 178 & 627 & 314 & 115,38 & 15 & 240 & 606 & 394 & 113,10 & 0,037 \\
\hline PPT M. vastus lateralis & 15 & 163 & 576 & 276 & 101,28 & 15 & 245 & 744 & 444 & 144,89 & 0,000 \\
\hline PPT M. tibialis anterior & 15 & 121 & 526 & 337 & 116,47 & 15 & 254 & 640 & 452 & 121,62 & 0,015 \\
\hline PPT Thenar & 15 & 170 & 473 & 310 & 90,30 & 15 & 204 & 592 & 401 & 112,08 & 0,033 \\
\hline
\end{tabular}

Tabelle 9: QST-Geschlechtsunterschiede innerhalb der Koxarthrosegruppe,

${ }^{*}$ Geschlechtsunterschied, T-Test: PPT, Mann-Whitney U Test: Thermale Schwellenwerte 


\begin{tabular}{|c|c|c|c|c|c|c|c|c|c|c|c|}
\hline \multirow{2}{*}{ Nicht betroffene Seite } & \multicolumn{5}{|c|}{ Gesunde Frauen } & \multicolumn{6}{|c|}{ Gesunde Männer } \\
\hline & $\mathrm{n}$ & Min. & Max. & MW & SD & $\mathrm{n}$ & Min. & Max. & MW & SD & $\mathrm{p}$ \\
\hline \multicolumn{12}{|c|}{ Thermale Schwellenwerte $\left({ }^{\circ} \mathrm{C}\right)$} \\
\hline CDT Thenar & 9 & 28,6 & 31,4 & 30,1 & 0,86 & 9 & 27,3 & 30,8 & 29,3 & 1,33 & 0,258 \\
\hline WDT Thenar & 9 & 32,7 & 35,1 & 33,6 & 0,78 & 9 & 33,1 & 36,6 & 34,2 & 1,27 & 0,340 \\
\hline СРT Thenar & 9 & 5,6 & 22,4 & 15,9 & 6,23 & 9 & 0,0 & 22,6 & 11,0 & 8,58 & 0,297 \\
\hline HPT Thenar & 9 & 36,0 & 45,4 & 40,2 & 3,62 & 9 & 36,3 & 50,5 & 44,0 & 5,03 & 0,113 \\
\hline CDT Trochanter major & 9 & 26,6 & 30,8 & 29,1 & 1,41 & 9 & 26,1 & 30,4 & 29,0 & 1,44 & 0,796 \\
\hline WDT Trochanter major & 9 & 33,4 & 37,9 & 35,3 & 1,27 & 9 & 34,1 & 37,8 & 35,8 & 1,46 & 0,730 \\
\hline CPT Trochanter major & 9 & 0,0 & 25,5 & 14,3 & 10,70 & 9 & 0,0 & 25,6 & 12,2 & 10,38 & 0,730 \\
\hline HPT Trochanter major & 9 & 36,6 & 48,5 & 42,9 & 3,84 & 9 & 38,8 & 49,2 & 45,3 & 3,49 & 0,161 \\
\hline \multicolumn{12}{|c|}{ Druckschmerzschwellen (kPa) } \\
\hline PPT Trochanter major & 9 & 477 & 654 & 547 & 63,91 & 9 & 469 & 883 & 654 & 146,38 & 0,113 \\
\hline PPT M. gluteus medius & 9 & 386 & 628 & 444 & 84,60 & 9 & 483 & 846 & 671 & 128,88 & 0,001 \\
\hline PPT M. vastus medialis & 9 & 278 & 421 & 353 & 48,26 & 9 & 301 & 821 & 501 & 166,64 & 0,031 \\
\hline PPT M. vastus lateralis & 9 & 216 & 423 & 315 & 62,79 & 9 & 302 & 639 & 441 & 98,08 & 0,004 \\
\hline PPT M. tibialis anterior & 9 & 334 & 586 & 488 & 88,27 & 9 & 416 & 799 & 607 & 159,10 & 0,222 \\
\hline PPT Thenar & 9 & 361 & 512 & 449 & 58,09 & 9 & 419 & 671 & 540 & 92,89 & 0,040 \\
\hline
\end{tabular}

Betroffene Seite

n Min. Max. MW SD n Min. Max. MW SD

$\mathrm{p}$

Thermale Schwellenwerte $\left({ }^{\circ} \mathrm{C}\right)$

\begin{tabular}{|c|c|c|c|c|c|c|c|c|c|c|c|}
\hline CDT Thenar & 9 & 29,0 & 31,1 & 30,2 & 0,61 & 9 & 27,7 & 31,2 & 29,8 & 1,10 & 0,436 \\
\hline WDT Thenar & 9 & 33,1 & 34,6 & 33,7 & 0,60 & 9 & 33,2 & 36,4 & 34,3 & 1,20 & 0,222 \\
\hline CPT Thenar & 9 & 3,6 & 26,6 & 17,0 & 6,58 & 9 & 0,0 & 22,7 & 11,3 & 8,25 & 0,258 \\
\hline HPT Thenar & 9 & 34,8 & 46,8 & 41,4 & 4,14 & 9 & 34,6 & 47,2 & 42,4 & 3,87 & 0,605 \\
\hline CDT Trochanter major & 9 & 25,2 & 31,0 & 28,4 & 2,17 & 9 & 25,5 & 30,9 & 28,9 & 1,51 & 0,666 \\
\hline WDT Trochanter major & 9 & 33,4 & 36,4 & 34,7 & 1,04 & 9 & 33,8 & 37,4 & 35,5 & 1,30 & 0,222 \\
\hline CPT Trochanter major & 9 & 0,0 & 25,1 & 13,5 & 11,99 & 9 & 0,0 & 23,3 & 15,3 & 9,03 & 0,730 \\
\hline HPT Trochanter major & 9 & 36,3 & 48,3 & 42,8 & 3,64 & 9 & 40,0 & 49,5 & 46,1 & 3,06 & 0,031 \\
\hline \multicolumn{12}{|c|}{ Druckschmerzschwellen (kPa) } \\
\hline PPT Trochanter major & 9 & 374 & 627 & 529 & 81,47 & 9 & 412 & 885 & 653 & 163,75 & 0,136 \\
\hline PPT M. gluteus medius & 9 & 346 & 673 & 446 & 116,41 & 9 & 473 & 817 & 642 & 123,07 & 0,004 \\
\hline PPT M. vastus medialis & 9 & 232 & 456 & 358 & 70,10 & 9 & 348 & 698 & 490 & 128,78 & 0,014 \\
\hline PPT M. vastus lateralis & 9 & 232 & 390 & 313 & 56,37 & 9 & 299 & 675 & 464 & 111,70 & 0,004 \\
\hline PPT M. tibialis anterior & 9 & 405 & 560 & 475 & 66,51 & 9 & 419 & 816 & 619 & 161,48 & 0,050 \\
\hline PPT Thenar & 9 & 347 & 576 & 448 & 65,10 & 9 & 289 & 684 & 517 & 121,48 & 0,136 \\
\hline
\end{tabular}

Tabelle 10: QST-Geschlechtsunterschiede innerhalb der Kontrollgruppe,

*Geschlechtsunterschied, T-Test: PPT, Mann-Whitney U Test: Thermale Schwellenwerte 


\subsection{Fragebögen}

\subsubsection{Oxford Hip Score}

Bitte beantworten Sie die folgenden 12 Fragen, indem Sie bei jeder Frage die zutreffende Zahl ankreuzen. Wählen Sie nur eine Antwort pro Frage.

Während der letzten 4 Wochen...

1. Wie würden Sie die Schmerzen beschreiben, die Sie üblicherweise in Ihrer Hüfte hatten?

Keine

Sehr Gering

Gering

Mäßig

Stark

2. Hatten Sie wegen Ihrer Hüfte Schwierigkeiten, sich selbst zu waschen und abzutrocknen (am ganzen Körper)?

$\square$ Überhaupt keine Schwierigkeiten

Sehr geringe Schwierigkeiten

Mäßige Schwierigkeiten

Extreme Schwierigkeit

$\square$ Unmöglich zu tun

3. Hatten Sie wegen Ihrer Hüfte Schwierigkeiten, in ein, bzw. aus einem Auto zu steigen oder öffentliche Verkehrsmittel zu benutzen? (welches Sie eher benutzen)

Überhaupt keine Schwierigkeiten

Sehr geringe Schwierigkeiten

Mäßige Schwierigkeiten

$\square$ Extreme Schwierigkeit

Unmöglich zu tun 
4. Konnten Sie sich ein Paar Socken, Strümpfe oder Strumpfhosen anziehen?

Ja, leicht

Mit geringen Schwierigkeiten

Mit mäßigen Schwierigkeiten

Mit extremen Schwierigkeiten

Nein, unmöglich

5. Konnten Sie die Haushaltseinkäufe selbst erledigen?

$\square$ Ja, leicht

$\square$ Mit geringen Schwierigkeiten

$\square$ Mit mäßigen Schwierigkeiten

$\square$ Mit extremen Schwierigkeiten

$\square$ Nein, unmöglich

6. Wie lange konnten Sie gehen, bevor Sie starke Schmerzen in Ihrer Hüfte bekamen (mit oder ohne Stock)?

Keine Schmerzen $>30$ Minuten

16 bis 30 Minuten

5 bis 15 Minuten

Nur zu Hause

$\square$ Gar nicht

7. Konnten Sie eine Treppe hinauf gehen?

$\square$ Ja, leicht

$\square$ Mit geringen Schwierigkeiten

$\square$ Mit mäßigen Schwierigkeiten

$\square$ Mit extremen Schwierigkeiten

$\square$ Nein, unmöglich 
8. Wie schmerzhaft war es für Sie wegen Ihrer Hüfte, nach einer Mahlzeit wieder vom Tisch aufzustehen?

Gar nicht schmerzhaft

Ein wenig schmerzhaft

Mäßig schmerzhaft

Sehr schmerzhaft

$\square$ Unerträglich

9. Haben Sie wegen Ihrer Hüfte beim Gehen gehinkt?

$\square$ Selten/nie

$\square$ Manchmal oder nur am Anfang

$\square$ Oft, nicht nur am Anfang

$\square$ Die meiste Zeit

$\square$ Die ganze Zeit

10. Hatten Sie plötzliche, starke Schmerzen - „einschiessend“, ,,stechend“ oder ,,krampfartig“ - in Ihrer betroffenen Hüfte?

$\square \mathrm{Nie}$

$\square$ Nur 1 oder 2 Tage

$\square$ Einige Tage

$\square$ Die meisten Tage

$\square$ Jeden Tag 
11. Wie sehr haben Schmerzen in Ihrer Hüfte Ihre normale Arbeit (einschließlich Hausarbeit) beeinträchtigt?

$\square$ Gar nicht

$\square$ Ein wenig

口 Mäßig

$\square$ Erheblich

$\square$ Vollständig

12. Wurden Sie nachts im Bett durch Schmerzen in Ihrer Hüfte gestört?

$\square \mathrm{Nie}$

$\square$ Nur 1 oder 2 Nächte

$\square$ Einige Nächte

$\square$ Die meisten Nächte

$\square$ Jede Nacht 


\subsubsection{Fibromyalgie-Syndrom Beschwerdefragebogen (FMS PSD)}

I. Bitte geben Sie an, wie ausgeprägt die folgenden Beschwerden in der letzten Woche bei Ihnen waren, in dem Sie das entsprechende Kästchen ankreuzen.

0: Nicht vorhanden

1: Geringfügige oder mild ausgeprägt; im Allgemeinen gering und/oder gelegentlich auftretend

2: Mäßige oder deutlich ausgeprägt; oft vorhanden und/oder mäßige Intensität

3: Stark ausgeprägt: ständig vorhandene, lebensbeeinträchtigende Beschwerden

Tagesmüdigkeit

Probleme beim Denken oder Gedächtnis

Morgenmüdigkeit müde (nicht erholsamer Schlaf) $\square 0 \square 1 \square 2 \square 3$

$\square 0 \square 1 \square 2 \square 3$

$\square 0 \square 1 \square 2 \square 3$

II. Wurden Sie in den letzten 6 Monaten durch eines der folgenden Symptome geplagt?

Schmerzen oder Krämpfe im Unterbauch: $\quad \square \mathrm{Ja}(1) \quad \square$ Nein (0)

Depression: $\quad \square \mathrm{Ja}(1) \quad \square \mathrm{Nein}(0)$

Kopfschmerz:

$\square \mathrm{Ja}(1) \quad \square \mathrm{Nein}(0)$

III. Bitte geben Sie an, ob Sie in den letzten 7 Tagen Schmerzen oder

Berührungsempfindlichkeit in den unten aufgeführten Körperregionen hatten.

Bitte kreuzen Sie das jeweilige Kästchen an, wenn diese Körperregion schmerzhaft oder druckempfindlich ist. Bitte bewerten Sie die rechte und linke Seite getrennt. 


\begin{tabular}{|l|l|l|}
\hline$\square$ Schulter, links & $\square$ Oberschenkel, links & $\square$ Kreuz \\
$\square$ Schulter, rechts & $\square$ Oberschenkel, rechts & $\begin{array}{l}\square \text { Oberer Rücken } \\
\text { (Brustwirbelsäule) } \\
\square \text { Nacken }\end{array}$ \\
\hline $\begin{array}{l}\text { Hüfte, links } \\
\square \text { Hüfte, rechts }\end{array}$ & $\begin{array}{l}\square \text { Unterschenkel, links } \\
\square \text { Unterschenkel, rechts }\end{array}$ & \\
$\square$ Oberarm, links & $\square$ Kiefer, links \\
$\square$ Oberarm, rechts & $\square$ Kiefer, rechts & \\
$\square$ Unterarm, links & $\square$ Brustkorb & Körperregionen Schmerzen \\
$\square$ Bauch & \\
\hline Unterarm, rechts & $\square$ & \\
\hline
\end{tabular}

IV. Waren die Beschwerden, die in den Fragen I-III aufgeführt sind, in der Regel in den letzten 3 Monaten vorhanden? $\square \mathrm{Ja} \quad \square$ Nein 


\subsubsection{Schmerzakzeptanz-Fragebogen (CPAQ)}

Ich stimme zu....

1) Ich komme mit meinen täglichen Aufgaben klar, egal wie stark meine Schmerzen sind.

nie fast nie selten manchmal häufig fast immer immer

$\begin{array}{lllllll}\square & \square & \square & \square & \square & \square & \square\end{array}$

2) Mein Leben verläuft gut, auch wenn ich an chronischen Schmerzen leide.

nie fast nie selten manchmal häufig fast immer immer

$\begin{array}{lllllll}\square & \square & \square & \square & \square & \square & \square\end{array}$

3) Es ist in Ordnung Schmerzen zu erleben.

nie fast nie selten manchmal häufig fast immer immer

$\begin{array}{llllll}\square & \square & \square & \square & \square & \square\end{array}$

4) Ich würde liebend gerne Dinge, die mir wichtig sind, opfern, wenn ich dann meine Schmerzen besser kontrollieren könnte.

nie fast nie selten manchmal häufig fast immer immer

$\begin{array}{lllllll}\square & \square & \square & \square & \square & \square & \square\end{array}$

5) Es ist für mich nicht notwendig, meine Schmerzen im Griff zu haben, um mein Leben gut bewältigen zu können.

nie fast nie selten manchmal häufig fastimmer immer

$\begin{array}{lllllll}\square & \square & \square & \square & \square & \square & \square\end{array}$

6) Auch wenn es Veränderungen gegeben hat, führe ich trotz meiner chronischen Schmerzen ein normales Leben.

nie fast nie selten manchmal häufig fastimmer immer

$\begin{array}{ccccccc} & \square & \square & \square & \square & \square & \square\end{array}$

7) Ich muss mich darauf konzentrieren, meine Schmerzen los zu werden.

nie fast nie selten manchmal häufig fastimmer immer

$\begin{array}{lllllll}\square & \square & \square & \square & \square & \square & \square\end{array}$

8) Es gibt viele Aktivitäten, denen ich nachgehe, wenn ich Schmerzen habe.

nie fast nie selten manchmal häufig fastimmer immer

$\begin{array}{llllll}\square & \square & \square & \square & \square & \square\end{array}$


9) Obwohl ich an chronischen Schmerzen leide, führe ich ein erfülltes Leben.

nie fast nie selten manchmal häufig fast immer immer

$\begin{array}{ccccccc} & \square & \square & \square & \square & \square & \square\end{array}$

10) Meine anderen Lebensziele sind mir alle wichtiger als die Kontrolle meiner Schmerzen.

nie fast nie selten manchmal häufig fastimmer immer

$\begin{array}{lllllll}\square & \square & \square & \square & \square & \square & \square\end{array}$

11) Bevor ich wichtige Veränderungen in meinem Leben vornehmen kann, müssen sich meine Gedanken und Gefühle dem Schmerz gegenüber verändern.

nie fast nie selten manchmal häufig fast immer immer

$\begin{array}{lllllll}\square & \square & \square & \square & \square & \square & \square\end{array}$

12) Trotz der Schmerzen verfolge ich inzwischen einen bestimmten Lebensweg.

nie fast nie selten manchmal häufig fast immer immer

$\begin{array}{lllllll}\square & \square & \square & \square & \square & \square & \square\end{array}$

13) Immer, wenn ich etwas mache, hat die Kontrolle meiner Schmerzen erste Priorität.

nie fast nie selten manchmal häufig fast immer immer

$\begin{array}{llllll}\square & \square & \square & \square & \square & \square\end{array}$

14) Bevor ich irgendwelche ernsthaften Pläne schmieden kann, muss ich zunächst etwas Kontrolle über meine Schmerzen gewinnen.

nie fast nie selten manchmal häufig fastimmer immer

$\begin{array}{lllllll}\square & \square & \square & \square & \square & \square & \square\end{array}$

15) Auch wenn meine Schmerzen stärker werden, kann ich meinen Verpflichtungen immer noch nachkommen.

nie fast nie selten manchmal häufig fast immer immer

$\begin{array}{llllll}\square & \square & \square & \square & \square & \square\end{array}$

16) Ich würde mein Leben besser im Griff haben, wenn ich meine negativen Gedanken in Bezug auf die Schmerzen besser kontrollieren könnte.

nie fast nie selten manchmal häufig fast immer immer

$\begin{array}{ccccccc}\text { nie } & \text { fast nie } & \text { selten } & \text { manchmal } & \text { haufig } & \text { fast immer } & \text { immer } \\ \square & \square & \square & \square & \square & \square & \square\end{array}$


17) Ich vermeide es, mich in Situationen zu bringen, in denen meine Schmerzen schlimmer werden könnten.

nie fast nie selten manchmal häufig fastimmer immer

$\begin{array}{lllllll}\square & \square & \square & \square & \square & \square & \square\end{array}$

18) Meine Sorgen und Ängste darüber, was der Schmerz mit mir machen könnte, stimmen.

nie fast nie selten manchmal häufig fast immer immer

$\begin{array}{lllllll}\square & \square & \square & \square & \square & \square & \square\end{array}$

19) Es ist eine Erleichterung zu erkennen, dass ich an meinen Schmerzen nichts verändern muss, um mit meinem Leben klar zu kommen.

nie fast nie selten manchmal häufig fastimmer immer

$\begin{array}{lllllll}\square & \square & \square & \square & \square & \square & \square\end{array}$

20) Wenn ich Schmerzen habe, kostet es mich große Überwindung etwas zu machen.

nie fast nie selten manchmal häufig fast immer immer

$\begin{array}{lllllll}\square & \square & \square & \square & \square & \square\end{array}$




\subsection{4 painDETECT}

Wie würden Sie Ihren Schmerz, der von Ihrer Hüfte ausgeht, jetzt im Augenblick
einschätzen?
$\begin{aligned} & \text { kein } \\ & \text { Schmerz }\end{aligned}$

Wie stark war der stärkste Schmerz durch Ihre Hüfte in den letzten 3 Monaten?

\begin{tabular}{|c|c|c|c|c|c|c|c|c|c|c|c|c|}
\hline $\begin{array}{l}\text { kein } \\
\text { Schmerz }\end{array}$ & $\square$ & $\square$ & $\square$ & $\square$ & $\square$ & 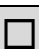 & $\square$ & $\square$ & $\square$ & $\square$ & $\square$ & $\begin{array}{r}\text { stärkster } \\
\text { vorstellbarer } \\
\text { Schmerz }\end{array}$ \\
\hline
\end{tabular}

Wie stark war der Schmerz durch Ihre Hüfte in den letzten 3 Monaten im

Durchschnitt?

\begin{tabular}{|c|c|c|c|c|c|c|c|c|c|c|c|c|}
\hline $\begin{array}{l}\text { kein } \\
\text { Schmerz }\end{array}$ & $\square$ & $\square$ & $\square$ & $\square$ & $\square$ & $\square$ & $\square$ & $\square$ & $\square$ & $\square$ & $\square$ & $\begin{array}{r}\text { stärkster } \\
\text { vorstellbarer } \\
\text { Schmerz }\end{array}$ \\
\hline
\end{tabular}

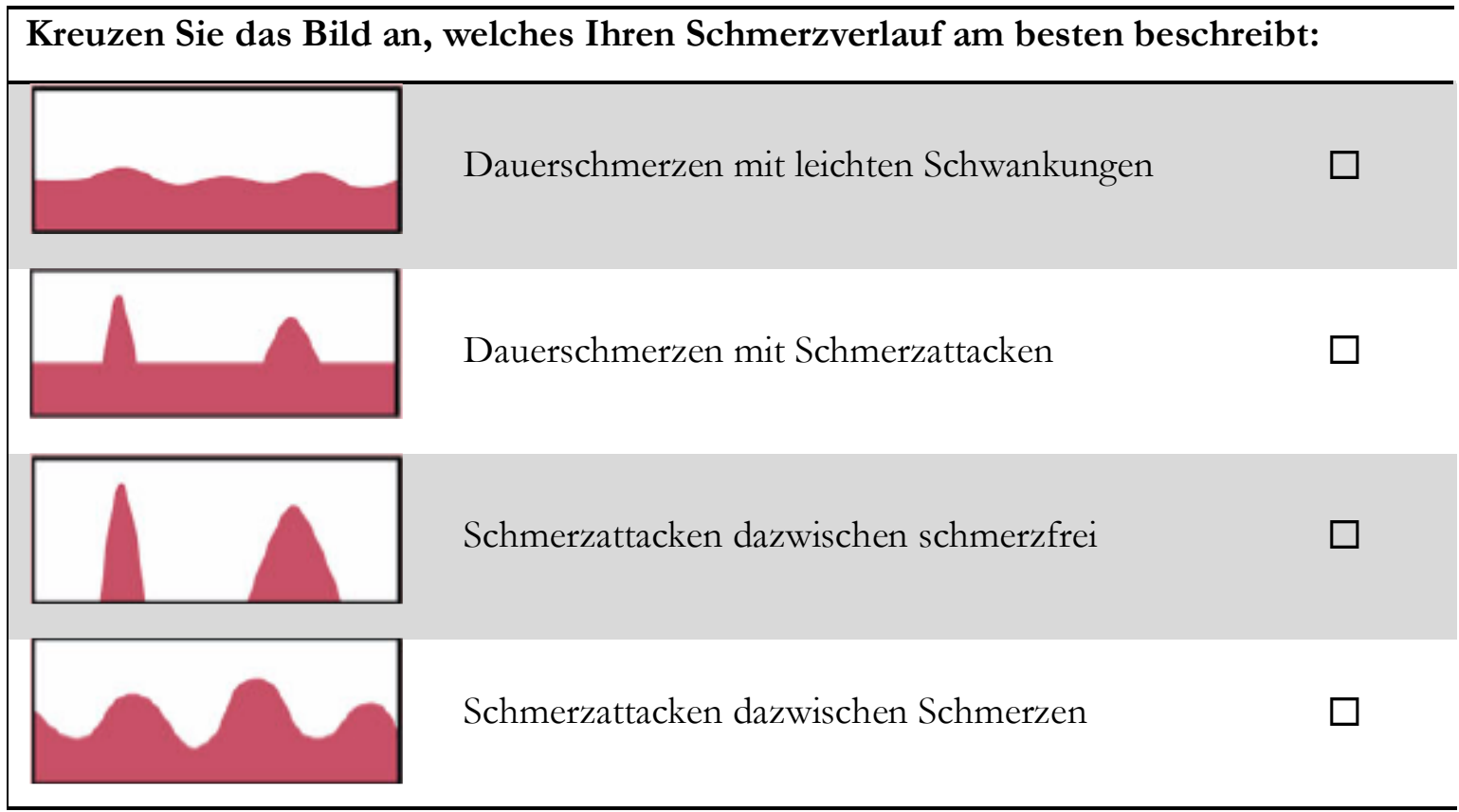




\section{Bitte kennzeichnen Sie Ihren Hauptschmerzbereich}

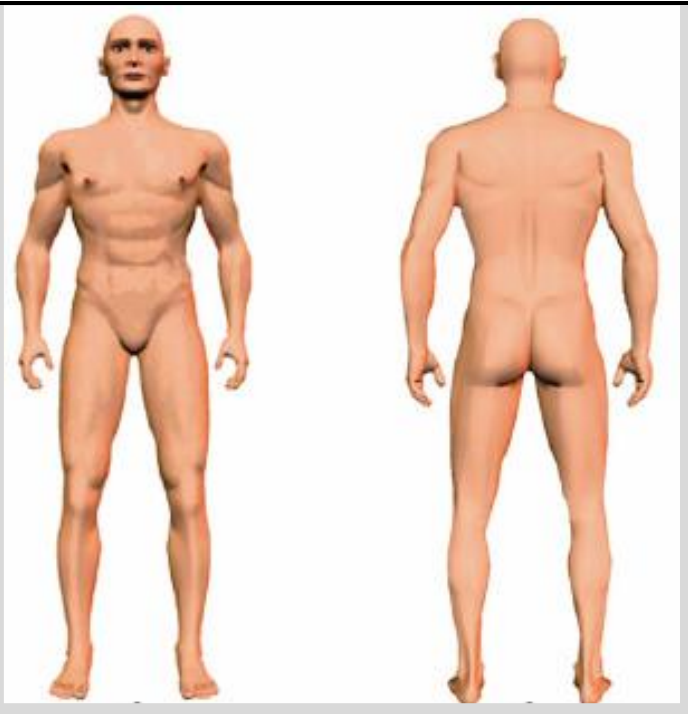

Strahlt Thr Schmerz in weitere

Körperregionen aus?

ja $\square$

nein $\square$

- wenn ja, dann zeichnen Sie bitte die Richtung ein, wohin der Schmerz ausstrahlt.

\begin{tabular}{lllllll}
\hline & nie & kaum & gering & mittel & stark & sehr \\
stark
\end{tabular}




\subsubsection{Lebensqualität (SF-36)}

(Bullinger et al. 1995)

In diesem Fragebogen geht es um Ihre Beurteilung Ihres Gesundheitszustandes. Der Bogen ermöglicht es, im Zeitverlauf nachzuvollziehen, wie Sie sich fühlen und wie Sie im Alltag zurechtkommen.

Bitte beantworten Sie jede der folgenden Fragen, indem Sie bei den Antwortmöglichkeiten die Zahl ankreuzen, die am besten auf Sie zutrifft.

1. Wie würden Sie Ihren Gesundheitszustand im Allgemeinen beschreiben?

(Bitte kreuzen Sie nur eine Zahl an)

Ausgezeichnet 1

Sehr gut 2

Gut 3

Weniger gut 4

Schlecht 5

2. Im Vergleich zum vergangenen Jahr, wie würden Sie Ihren derzeitigen

Gesundheitszustand beschreiben?

(Bitte kreuzen Sie nur eine Zahl an)

Derzeit viel besser als vor einem Jahr

1

Derzeit etwas besser als vor einem Jahr

Etwa so wie vor einem Jahr

Derzeit etwas schlechter als vor einem Jahr

4

Derzeit viel schlechter als vor einem Jahr

3. Im Folgenden sind einige Tätigkeiten beschrieben, die Sie vielleicht an einem normalen Tag ausüben. Sind Sie durch Ihren derzeitigen Gesundheitszustand bei diesen Tätigkeiten eingeschränkt? Wenn ja, wie stark?

(Bitte kreuzen Sie in jeder Zeile nur eine Zahl an)

\begin{tabular}{|c|c|c|c|c|}
\hline & TÄTIGKEITEN & $\begin{array}{c}\text { Ja, stark } \\
\text { eingeschränkt }\end{array}$ & $\begin{array}{c}\text { Ja, etwas } \\
\text { eingeschränkt }\end{array}$ & $\begin{array}{l}\text { Nein, über- } \\
\text { haupt nicht } \\
\text { eingeschränkt }\end{array}$ \\
\hline a) & $\begin{array}{l}\text { anstrengende Tätigkeiten, z.B. schnell laufen, } \\
\text { schwere Gegenstände heben, anstrengenden } \\
\text { Sport treiben }\end{array}$ & 1 & 2 & 3 \\
\hline b) & $\begin{array}{l}\text { mittelschwere Tätigkeiten, z.B. einen Tisch } \\
\text { verschieben, staubsaugen, kegeln, Golf, } \\
\text { spielen }\end{array}$ & 1 & 2 & 3 \\
\hline c) & Einkaufstaschen heben oder tragen & 1 & 2 & 3 \\
\hline d) & mehrere Treppenabsätze steigen & 1 & 2 & 3 \\
\hline e) & einen Treppenabsatz steigen & 1 & 2 & 3 \\
\hline f) & sich beugen, knien, bücken & 1 & 2 & 3 \\
\hline
\end{tabular}




\begin{tabular}{|l|l|c|c|c|}
\hline g) & mehr als 1 Kilometer zu Fuß gehen & 1 & 2 & 3 \\
\hline h) & $\begin{array}{l}\text { mehrere Straßenkreuzungen weit zu Fuß } \\
\text { gehen }\end{array}$ & 1 & 2 & 3 \\
\hline i) & eine Straßenkreuzung weit zu Fuß gehen & 1 & 2 & 3 \\
\hline j) & sich baden oder anziehen & 1 & 2 & 3 \\
\hline
\end{tabular}

4. Hatten Sie in den vergangenen vier Wochen aufgrund Ihrer körperlichen

Gesundheit irgendwelche Schwierigkeiten bei der Arbeit oder anderen alltäglichen Tätigkeiten im Beruf bzw. zu Hause?

(Bitte kreuzen Sie in jeder Zeile nur eine Zahl an)

\begin{tabular}{|c|l|c|c|}
\hline & SCHWIERIGKEITEN & Ja & Nein \\
\hline a) & Ich konnte nicht so lange wie üblich tätig sein & 1 & 2 \\
\hline b) & Ich habe weniger geschafft als ich wollte & 1 & 2 \\
\hline c) & Ich konnte nur bestimmte Dinge tun & 1 & 2 \\
\hline d) & $\begin{array}{l}\text { Ich hatte Schwierigkeiten bei der Ausführung (z.B.: ich musste mich } \\
\text { besonders anstrengen) }\end{array}$ & 1 & 2 \\
\hline
\end{tabular}

5. Hatten Sie in den vergangenen vier Wochen aufgrund seelischer Probleme irgendwelche Schwierigkeiten bei der Arbeit oder anderen alltäglichen Tätigkeiten im Beruf bzw. zu Hause (z.B. weil Sie sich niedergeschlagen oder ängstlich fühlten)? (Bitte kreuzen Sie in jeder Zeile nur eine Zahl an)

\begin{tabular}{|c|l|c|c|}
\hline & SCHWIERIGKEITEN & Ja & Nein \\
\hline a) & Ich konnte nicht so lange wie üblich tätig sein & 1 & 2 \\
\hline b) & Ich habe weniger geschafft als ich wollte & 1 & 2 \\
\hline c) & Ich konnte nicht so sorgfältig wie üblich arbeiten & 1 & 2 \\
\hline
\end{tabular}

6. Wie sehr haben Ihre körperliche Gesundheit oder seelische Probleme in den vergangenen vier Wochen Ihre normalen Kontakte zu Familienangehörigen, Freunden, Nachbarn oder im Bekanntenkreis beeinträchtigt?

(Bitte kreuzen Sie nur eine Zahl an)

$\begin{array}{ll}\text { Überhaupt nicht } & 1 \\ \text { Etwas } & 2 \\ \text { Mäßig } & 3 \\ \text { Ziemlich } & 4 \\ \text { Sehr } & 5\end{array}$


7. Wie stark waren Ihre Schmerzen in den vergangenen vier Wochen?

(Bitte kreuzen Sie nur eine Zahl an)

Ich hatte keine Schmerzen 1

Sehr leicht 2

Leicht 3

Mäßig 4

Stark 5

Sehr stark 6

8. Inwieweit haben die Schmerzen Sie in den vergangenen vier Wochen bei der Ausübung Ihrer Alltagstätigkeiten zu Hause und im Beruf behindert?

(Bitte kreuzen Sie nur eine Zahl an)

Überhaupt nicht 1

Ein bißchen 2

Mäßig 3

Ziemlich 4

Sehr 5

9. In diesen Fragen geht es darum, wie Sie sich fühlen und wie es Ihnen in den vergangenen vier Wochen gegangen ist. (Bitte kreuzen Sie in jeder Zeile die Zahl an, die Ihrem Befinden am ehesten entspricht). Wie oft waren Sie in den vergangenen vier Wochen...

(Bitte kreuzen Sie nur eine Zahl an)

\begin{tabular}{|l|c|c|c|c|c|c|}
\hline Befinden & Immer & Meistens & Ziemlich oft & Manchmal & Selten & Nie \\
\hline a)...voller Schwung? & 1 & 2 & 3 & 4 & 5 & 6 \\
\hline b)...sehr nervös? & 1 & 2 & 3 & 4 & 5 & 6 \\
\hline $\begin{array}{l}\text { c)...so niedergeschlagen, } \\
\text { dass Sie nichts aufheitern } \\
\text { konnte? }\end{array}$ & 1 & 2 & 3 & 4 & 5 & 6 \\
\hline $\begin{array}{l}\text { d)...ruhig und gelassen? } \\
\text { e)...voller Energie? }\end{array}$ & 1 & 2 & 3 & 4 & 5 & 6 \\
\hline $\begin{array}{l}\text { f)...entmutigt und traurig? } \\
\text { g)...erschöpft? }\end{array}$ & 1 & 2 & 3 & 4 & 5 & 6 \\
\hline h)...glücklich? & 1 & 2 & 3 & 4 & 5 & 6 \\
\hline \begin{tabular}{l} 
i)...müde? \\
\hline
\end{tabular} & 1 & 2 & 3 & 4 & 5 & 6 \\
\hline
\end{tabular}

10. Wie häufig haben Ihre körperliche Gesundheit oder seelische Probleme in den vergangenen vier Wochen Ihre Kontakte zu anderen Menschen (Familienangehörigen, Freunden, Nachbarn oder im Bekanntenkreis) beeinträchtigt? (Bitte kreuzen Sie nur eine Zahl an) 


$\begin{array}{ll}\text { Immer } & 1 \\ \text { Meistens } & 2 \\ \text { Manchmal } & 3 \\ \text { Selten } & 4 \\ \text { Nie } & 5\end{array}$

11. Inwieweit trifft jede der folgenden Aussagen auf Sie zu? (Bitte kreuzen Sie in jeder Zeile nur eine Zahl an)

\begin{tabular}{|c|c|c|c|c|c|}
\hline Aussagen & Trifft ganz zu & $\begin{array}{c}\text { Trifft } \\
\text { weitgehend } \\
\mathrm{zu}\end{array}$ & Weiß nicht & $\begin{array}{c}\text { Trifft } \\
\text { weitgehend } \\
\text { nicht zu }\end{array}$ & $\begin{array}{c}\text { Trifft } \\
\text { überhaupt nicht } \\
\text { zu }\end{array}$ \\
\hline $\begin{array}{l}\text { a) Ich scheine } \\
\text { etwas leichter } \\
\text { als andere krank } \\
\text { zu werden. }\end{array}$ & 1 & 2 & 3 & 4 & 5 \\
\hline $\begin{array}{l}\text { b) Ich bin } \\
\text { genauso gesund } \\
\text { wie alle anderen, } \\
\text { die ich kenne. }\end{array}$ & 1 & 2 & 3 & 4 & 5 \\
\hline $\begin{array}{l}\text { c) Ich erwarte, } \\
\text { dass meine } \\
\text { Gesundheit } \\
\text { nachlässt. }\end{array}$ & 1 & 2 & 3 & 4 & 5 \\
\hline $\begin{array}{l}\text { Ich erfreue mich } \\
\text { ausgezeichneter } \\
\text { Gesundheit }\end{array}$ & 1 & 2 & 3 & 4 & 5 \\
\hline
\end{tabular}




\subsubsection{Pain Catastrophizing Scale}

Die folgenden dreizehn Sätze beschreiben verschiedene Gedanken und Gefühle, die bei Schmerzen

auftreten können. Bitte markieren Sie auf der folgenden Skala bei jeder Frage, wie stark diese Gedanken und Gefühle auf Sie zutreffen, wenn Sie Schmerzen haben.

Bewertung:

$0=$ trifft überhaupt nicht zu, $1=$ trifft eher nicht zu, $2=$ teils-teils, $3=$ trifft eher zu, $4=$ trifft immer zu

1. Ich mache mir ständig Sorgen, ob die Schmerzen wohl jemals wieder aufhören werden? $\left|\_0 \_\right| \_1 \_\left|-2 \_\right| \_3-\left|\_4-\right|$

2. Ich denke, ich kann nicht mehr.

$\left|\_0-\right| \_1 \_\left|-2 \_\right|-3-\left|-4 \_\right|$

3. Der Zustand ist schrecklich und ich denke, dass es nie mehr besser wird.

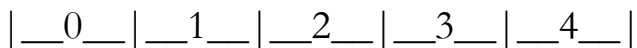

4. Der Zustand ist furchtbar und droht mich zu überwältigen.

$\mid$

5. Ich habe das Gefühl, ich halte es nicht mehr aus.

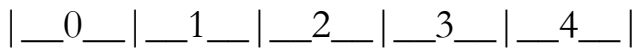

6. Ich bekomme Angst, dass die Schmerzen noch stärker werden.

$|-0-|-1-|-2-|{ }^{3}-|-4-|$

7. Ich denke ständig an andere Situationen, in denen ich Schmerzen hatte.

$\mid$

8. Ich wünsche mir verzweifelt, dass die Schmerzen weggehen.

$\mid$

9. Ich kann nicht aufhören, an die Schmerzen zu denken.

$\mid$

10. Ich denke ständig daran, wie sehr es schmerzt.

$\mid$

11. Ich denke ständig daran, wie sehr ich mir ein Ende der Schmerzen herbeiwünsche.

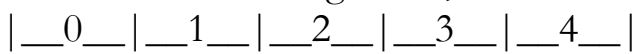

12. Es gibt nicht was ich tun kann, um die Schmerzen zu lindern.

$|-0-|-1-|-2-|-3-|-4-|$

13. Ich mache mir Sorgen, dass die Schmerzen auf etwas Schlimmes hindeuten.

$\mid{ }_{\text {Kathrin }} 0 \ldots$

Kathrin Meyer et. al. Cross-cultural adaption, reliability and validity of the German version of the Pain Catastrophizing Scale. Journal of

Psychosomatic Research 64 (2008): 469-478 


\subsubsection{TSK-DE}

Mit den nachfolgenden Fragen möchten wir untersuchen, wie Sie selbst zu Ihren Schmerzen stehen.

Bitte geben Sie an, in welchem Maß Sie der vorgegebenen Aussage zustimmen. Bitte kreuzen Sie dafür bei jeder Frage das entsprechende Kästchen an.

(Ein Kreuz pro Frage.)

1. Ich habe Angst davor, dass ich mich möglicherweise verletze, wenn ich Sport treibe.

$\square$ stimmt überhaupt nicht $\square$ eher nein $\square$ eher ja $\square$ stimmt völlig

2. Wenn ich versuchen würde, mich über die Schmerzen hinweg zu setzen, würden sie noch schlimmer.

$\square$ stimmt überhaupt nicht $\square$ eher nein $\quad \square$ eher ja $\square$ stimmt völlig

3. Mein Körper sagt mir, dass ich etwas sehr Schlimmes habe.

$\square$ stimmt überhaupt nicht $\square$ eher nein $\quad \square$ eher ja $\square$ stimmt völlig

4. Meine Schmerzen würden vermutlich gelindert werden, wenn ich Sport treiben würde.

$\square$ stimmt überhaupt nicht $\square$ eher nein $\quad \square$ eher ja $\quad \square$ stimmt völlig

5. Mein Gesundheitszustand wird von anderen nicht ernst genug genommen.

$\square$ stimmt überhaupt nicht $\quad \square$ eher nein $\quad \square$ eher ja $\quad \square$ stimmt völlig

6. Wegen des Schmerzproblems ist mein Körper für den Rest meines Lebens gefährdet.

$\square$ stimmt überhaupt nicht $\square$ eher nein $\quad \square$ eher ja $\square$ stimmt völlig

7. Schmerz bedeutet immer, dass ich mich verletzt habe.

$\square$ stimmt überhaupt nicht $\square$ eher nein $\quad \square$ eher ja $\square$ stimmt völlig

8. Nur weil etwas meine Schmerzen verstärkt, bedeutet das nicht, dass es gefährlich ist.

$\square$ stimmt überhaupt nicht $\square$ eher nein $\quad \square$ eher ja $\square$ stimmt völlig

9. Ich habe Angst, dass ich mich versehentlich verletzen könnte.

$\square$ stimmt überhaupt nicht $\square$ eher nein $\square$ eher ja $\square$ stimmt völlig 
10. Die sicherste Art, zu verhindern, dass meine Schmerzen schlimmer werden, ist einfach darauf zu achten, dass ich keine unnötigen Bewegungen mache.

$\square$ stimmt überhaupt nicht $\quad \square$ eher nein $\quad \square$ eher ja $\square$ stimmt völlig

11. Ich hätte nicht so viel Schmerzen, wenn nicht etwas Bedenkliches in meinem Körper vor sich ginge.

$\square$ stimmt überhaupt nicht $\square$ eher nein $\quad \square$ eher ja $\square$ stimmt völlig

12. Auch wenn ich Schmerzen habe, würde es mir besser gehen, wenn ich körperlich aktiv wäre.

$\square$ stimmt überhaupt nicht $\square$ eher nein $\quad \square$ eher ja $\square$ stimmt völlig

13. Meine Schmerzen sagen mir, wann ich mit dem Training aufhören muss, um mich nicht zu verletzen.

$\square$ stimmt überhaupt nicht $\quad \square$ eher nein $\quad \square$ eher ja $\quad \square$ stimmt völlig

14. Für jemand in meinem Gesundheitszustand ist es wirklich nicht ratsam, körperlich aktiv zu sein.

$\square$ stimmt überhaupt nicht $\square$ eher nein $\quad \square$ eher ja $\square$ stimmt völlig

15. Ich kann nicht all die Dinge tun, die gesunde Menschen machen, da ich mich zu leicht verletzen könnte.

$\square$ stimmt überhaupt nicht $\square$ eher nein $\quad \square$ eher ja $\square$ stimmt völlig

16. Auch wenn mir etwas starke Schmerzen bereitet, denke ich nicht, dass es tatsächlich gefährlich ist.

$\square$ stimmt überhaupt nicht $\square$ eher nein $\quad \square$ eher ja $\square$ stimmt völlig

17. Niemand sollte Sport treiben müssen, wenn er / sie Schmerzen hat.

$\square$ stimmt überhaupt nicht $\square$ eher nein $\quad \square$ eher ja $\square$ stimmt völlig

Tampa Scale of Kinesiophobia - Deutsche Version, () Abt. für Medizinische Psychologie, Ruhr-Universität Bochum 


\subsubsection{Von Korff Scale (VKS):}

(Von Korff et al. 1992)

\section{Frage 1:}

An ungefähr wie vielen Tagen konnten Sie in den letzten drei Monaten aufgrund Ihrer Schmerzen Ihren normalen Beschäftigungen (Beruf, Schule/Studium, Hausarbeit) nicht nachgehen?

\section{Tage}

In den folgenden Fragen 2 bis 4 geht es um die Stärke Ihrer Schmerzen.Sie können die Angaben jeweils auf einer Skala von 0 - 10 abstufen.

Der Wert 0 bedeutet, daß Sie keine Schmerzen haben/hatten, der Wert 10 bedeutet, daß die Schmerzen nicht schlimmer sein könnten. Mit den dazwischen liegenden Werten können Sie Abstufungen vornehmen.

Frage 2:

Wie würden Sie Ihre Schmerzen, wie sie in diesem Augenblick sind, einstufen?

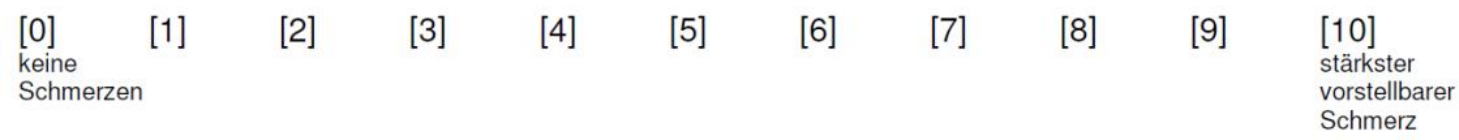

\section{Frage 3:}

Wenn Sie an die Tage denken, an denen Sie in den letzten drei Monaten Schmerzen hatten, wie würden Sie Ihre stärksten Schmerzen einstufen?

\begin{tabular}{|c|c|c|c|c|c|c|c|c|c|}
\hline $\begin{array}{l}{[0] \quad[1]} \\
\text { keine } \\
\text { Schmerzen }\end{array}$ & [2] & [3] & [4] & [5] & [6] & [7] & [8] & [9] & $\begin{array}{l}{[10]} \\
\text { stärkster } \\
\text { vorstellbarer } \\
\text { Schmerz }\end{array}$ \\
\hline
\end{tabular}

Frage 4:

Wenn Sie an die Tage denken, an denen Sie in den letzten drei Monaten Schmerzen hatten, wie würden Sie die durchschnittliche Stärke der Schmerzen einstufen?

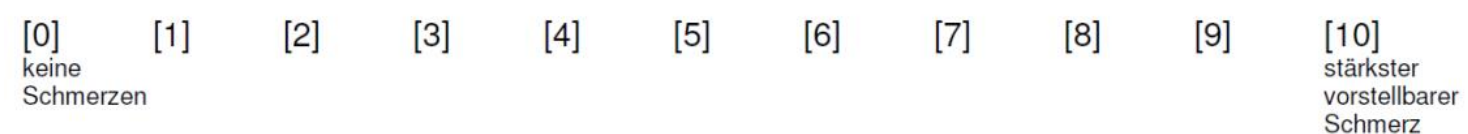


Im folgenden (Fragen 5 bis 7) geht es um die Beeinträchtigung von Aktivitäten durch Schmerzen. Sie können Ihre Angaben jeweils auf einer Skala von 0 bis 10 abstufen. Der Wert 0 bedeutet keine Beeinträchtigung, der Wert 10 bedeutet, dass Sie außerstande sind/waren, irgendetwas zu tun. Mit den dazwischen liegenden Werten können Sie Abstufungen vornehmen.

Frage 5:

Inwieweit haben Ihre Schmerzen Sie in den letzten drei Monaten bei Ihren alltäglichen Beschäftigungen beeinträchtigt?

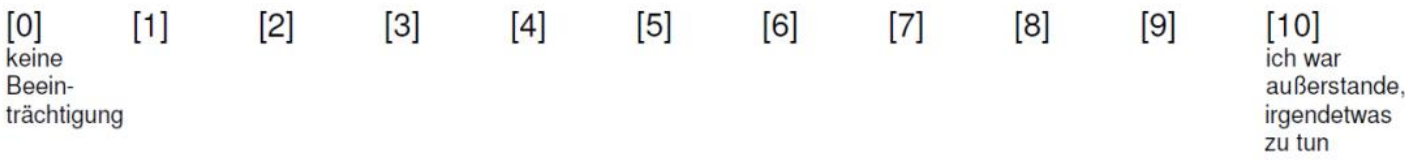

Frage 6:

Inwieweit haben in den letzten drei Monaten die Schmerzen Ihre Fähigkeit, an Familienoder Freizeitaktivitäten teilzunehmen, beeinträchtigt?

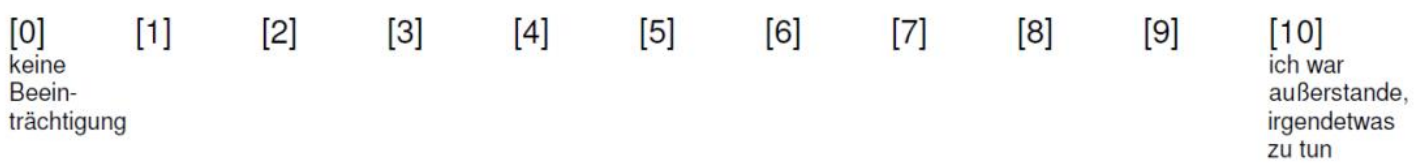

Frage 7:

Und inwieweit haben in den letzten drei Monaten die Schmerzen Ihre Fähigkeit beeinträchtigt, Ihre Arbeit/Hausarbeit zu verrichten?

$\begin{array}{llllllllll}{[0]} & {[1]} & {[2]} & {[3} & {[4]} & {[5]} & {[6]} & {[7]} & {[8]} & {[9] \quad \begin{array}{l}\text { [10] } \\ \text { ich war } \\ \text { aukerstande, } \\ \text { irgendetwas } \\ \text { zeine tun }\end{array}} \\ \begin{array}{l}\text { Beein- } \\ \text { trächtigung }\end{array}\end{array}$




\subsubsection{Depression, Anxiety, Stress Scales (DASS)}

0 Traf gar nicht auf mich zu

1 Traf bis zu einem gewissen Grad auf mich zu oder manchmal

2 Traf in beträchtlichem Maße auf mich zu oder ziemlich oft

3 Traf sehr stark auf mich zu oder die meiste Zeit

1. Ich fand es schwer, mich zu beruhigen.

2. Ich spürte, dass mein Mund trocken war.

3. Ich konnte überhaupt keine positiven Gefühle mehr erleben

4. Ich hatte Atemprobleme (z.B. übermäßig schnelles Atmen, Atemlosigkeit ohne körperliche Anstrengung).

5. Es fiel mir schwer, mich dazu aufzuraffen, Dinge zu erledigen.

6. Ich tendierte dazu, auf Situationen überzureagieren.

7. Ich zitterte (z.B. an den Händen).

8. Ich fand alles anstrengend.

9. Ich machte mir Sorgen über Situationen, in denen ich in Panik geraten und mich lächerlich machen könnte.

10. Ich hatte das Gefühl, dass ich mich auf nichts mehr freuen konnte.

11. Ich bemerkte, dass ich mich schnell aufregte.

12. Ich fand es schwierig, mich zu entspannen.

13. Ich fühlte mich niedergeschlagen und traurig.

14. Ich reagierte ungehalten auf alles, was mich davon abhielt, meine momentane Tätigkeit fortzuführen.

15. Ich fühlte mich einer Panik nahe.

16. Ich war nicht in der Lage, mich für irgendetwas zu begeistern.

17. Ich fühlte mich als Person nicht viel wert.

18. Ich fand mich ziemlich empfindlich.

19. Ich habe meinen Herzschlag gespürt, ohne dass ich mich körperlich

angestrengt hatte (z.B. Gefühl von Herzrasen oder Herzstolpern).

20. Ich fühlte mich grundlos ängstlich.

21. Ich empfand das Leben als sinnlos.

\begin{tabular}{|c|c|c|c|c|}
\hline 0 & 1 & 2 & 3 & $\mathrm{~S}$ \\
\hline 0 & 1 & 2 & 3 & $\mathrm{~A}$ \\
\hline 0 & 1 & 2 & 3 & $\mathrm{D}$ \\
\hline
\end{tabular}

\begin{tabular}{|l|l|l|l|l} 
& & & & \\
0 & 1 & 2 & 3 & A
\end{tabular}

\begin{tabular}{|l|l|l|l|l|}
\hline & 1 & 2 & 3 & $\mathrm{~A}$ \\
\hline 0 & 1 & 2 & 3 & $\mathrm{D}$ \\
\hline
\end{tabular}

\begin{tabular}{|l|l|l|l|l|}
0 & 1 & 2 & 3 & $\mathrm{~S}$ \\
\hline 0 & 1 & 2 & 3 & A \\
\hline
\end{tabular}

\begin{tabular}{l|l|l|l|l|}
0 & 1 & 2 & 3 & $\mathrm{~s}$ \\
\hline
\end{tabular}

\begin{tabular}{l|l|l|l|l}
0 & 1 & 2 & 3 & A
\end{tabular}

\begin{tabular}{|l|l|l|l|l}
\hline 0 & 1 & 2 & 3 & $\mathrm{D}$
\end{tabular}

\begin{tabular}{|c|c|c|c|c|}
\hline 0 & 1 & 2 & 3 & $\mathrm{~s}$ \\
\hline 0 & 1 & 2 & 3 & $\mathrm{~s}$ \\
\hline 0 & 1 & 2 & 3 & $\mathrm{D}$ \\
\hline
\end{tabular}

\begin{tabular}{l|l|l|l|l}
0 & 1 & 2 & 3 & $\mathrm{~s}$
\end{tabular}

\begin{tabular}{l|l|l|l|l}
0 & 1 & 2 & 3 & A
\end{tabular}

\begin{tabular}{l|l|l|l|l}
0 & 1 & 2 & 3 & D
\end{tabular}

\begin{tabular}{l|l|l|l|l} 
& 1 & 2 & 3 & $\mathrm{D}$ \\
\hline 0 & 1 & 2 & 3 & $\mathrm{D}$
\end{tabular}

DASS (C) Nilges, Korb, Essau 2012, Diese Zeile bitte nicht ausfüllen: D: 


\section{$7 \quad$ Literaturverzeichnis}

Altman R, Alarcón G, Appelrouth D, Bloch D, Borenstein D, Brandt K, Brown C, Cooke TD, Daniel W, et al. (1991): The American College of Rheumatology criteria for the classification and reporting of osteoarthritis of the hip. Arthritis Rheum $\underline{34}, 505-514$

Altman R, Abadie E, Avouac B, Bouvenot G, Branco J, Bruyere O, Calvo G, Devogelaer J-P, Dreiser RL, Herrero-Beaumont G, et al. (2005): Total joint replacement of hip or knee as an outcome measure for structure modifying trials in osteoarthritis. Osteoarthritis Cartilage 13, $13-19$

Amaro A, Amado F, Duarte JA, Appell H-J (2007): Gluteus Medius Muscle Atrophy is Related to Contralateral and Ipsilateral Hip Joint Osteoarthritis. Int J Sports Med 28, 10351039

Amris K, Jespersen A, Bliddal H (2010): Self-reported somatosensory symptoms of neuropathic pain in fibromyalgia and chronic widespread pain correlate with tender point count and pressure-pain thresholds. Pain 151, 664-669

Arendt-Nielsen L, Eskehave TN, Egsgaard LL, Petersen KK, Graven-Nielsen T, Hoeck HC, Simonsen O, Siebuhr AS, Karsdal M, Bay-Jensen AC (2014): Association Between Experimental Pain Biomarkers and Serologic Markers in Patients With Different Degrees of Painful Knee Osteoarthritis. Arthritis Rheumatol 66, 3317-3326

Arnoldi J-C, Djurhuus J, Heerfordt J, Karle A (1980): Intraosseous Phlebography, Intraosseous Pressure Measurements and 99mTc-Polyphosphate Scintigraphy in Patients with Various Painful Conditions in the hip and Knee, Acta Orthop Scand, 51, 19-28

Barbero M, Moresi F, Leoni D, Gatti R, Egloff M, Falla D (2015): Test-retest reliability of pain extent and pain location using a novel method for pain drawing analysis. Eur J Pain 19, 1129-1138

Bartley EJ, King CD, Sibille KT, Cruz-Almeida Y, Riley JL, Glover TL, Goodin BR, Sotolongo AS, Herbert MS, Bulls HW, et al. (2016): Enhanced Pain Sensitivity Among Individuals With Symptomatic Knee Osteoarthritis: Potential Sex Differences in Central Sensitization. Arthritis Care Res 68, 472-480

Bennell KL, Rini C, Keefe F, French S, Nelligan R, Kasza J, Forbes A, Dobson F, Abbott JH, Dalwood A, et al. (2015): Effects of Adding an Internet-Based Pain Coping Skills Training Protocol to a Standardized Education and Exercise Program for People With Persistent Hip Pain (HOPE Trial): Randomized Controlled Trial Protocol. Phys Ther 25, 1408-1422

Bergman S, Herrström P, Jacobsson LT, Petersson IF (2002): Chronic widespread pain: a three year followup of pain distribution and risk factors. J Rheumatol 22, 818-825

Binder A, Tomforde M, Baron R (2013): Neuropathischer Schmerz. In: Baron R, Koppert W, Strumpf M, Willweber-Strumpf A: Praktische Schmerztherapie. 3. Auflage; Springer Medizin Verlag, Heidelberg 2013, 363-378 
Biurrun Manresa JA, Neziri AY, Curatolo M, Arendt-Nielsen L, Andersen OK (2011): Testretest reliability of the nociceptive withdrawal reflex and electrical pain thresholds after single and repeated stimulation in patients with chronic low back pain. Eur J Appl Physiol 111, 8392

Bobic V (1999): The utilisation of osteochondral autologous grafts in the treatment of articular cartilage lesions. Orthop 28, 19-25

Borys C, Lutz J, Strauss B, Altmann U (2015): Effectiveness of a Multimodal Therapy for Patients with Chronic Low Back Pain Regarding Pre-Admission Healthcare Utilization. PLoS ONE 10(11)

Brabant T, Stichtenoth D (2005): Medikamentöse Arthrosetherapie im Alter. Rheumatol 64, $467-472$

Breivik H, Collett B, Ventafridda V, Cohen R, Gallacher D (2006): Survey of chronic pain in Europe: Prevalence, impact on daily life, and treatment. Eur J Pain 10, 287-287

Breivik H, Eisenberg E, O’Brien T (2013): The individual and societal burden of chronic pain in Europe: the case for strategic prioritisation and action to improve knowledge and availability of appropriate care. BMC Public Health 13, 1229

Bruyère $\mathrm{O}$, Cooper $\mathrm{C}$, Arden N, Branco J, Brandi ML, Herrero-Beaumont G, Berenbaum F, Dennison E, Devogelaer J-P, Hochberg M, et al. (2015): Can We Identify Patients with High Risk of Osteoarthritis Progression Who Will Respond to Treatment? A Focus on Epidemiology and Phenotype of Osteoarthritis. Drugs Aging 32, 179-187

Bryner P (1994): Extent measurement in localised low-back pain: a comparison of four methods. Pain 59, 281-285

Bullinger M, Kirchberger I, Ware J (1995): Der deutsche SF-36 Health Survey Übersetzung und psychometrische Testung eines krankheitsübergreifenden Instruments zur Erfassung der gesundheitsbezogenen Lebensqualität. Gesundheitswissenschaften J Public Health 3, 21-36

Butler S, Landmark T, Glette M, Borchgrevink P, Woodhouse A (2016): Chronic widespread pain - the need for a standard definition. Pain 157, 541-543

Chappell AS, Ossanna MJ, Liu-seifert H, Iyengar S, Skljarevski V, Li LC, Bennett RM, Collins $\mathrm{H}$ (2009): Duloxetine, a centrally acting analgesic, in the treatment of patients with osteoarthritis knee pain: A 13-week, randomized, placebo-controlled trial. Pain 146, 253-260

Chiarotto A, Fernandez-de-las-Peñas C, Castaldo M, Negrini S, Villafañe JH (2013):

Widespread pressure pain hypersensitivity in elderly subjects with unilateral thumb carpometacarpal osteoarthritis. Hand 8, 422-429

Clauw DJ, Hassett AL (2017): The role of centralised pain in osteoarthritis. Clin Exp Rheumatol 35 Suppl 107, 79-84

Courtney CA, Lewek MD, Witte PO, Chmell SJ, Hornby TG (2009): Heightened Flexor Withdrawal Responses in Subjects With Knee Osteoarthritis. J Pain 10, 1242-1249 
Cross M, Smith E, Hoy D, Nolte S, Ackerman I, Fransen M, Bridgett L, Williams S, Guillemin F, Hill CL, et al. (2014): The global burden of hip and knee osteoarthritis: estimates from the Global Burden of Disease 2010 study. Ann Rheum Dis 73, 1323-1330

Cuesta-Vargas AI, Neblett R, Chiarotto A, Kregel J, Nijs J, van Wilgen CP, Pitance L, Knezevic A, Gatchel RJ, Mayer TG, et al. (2018): Dimensionality and Reliability of the Central Sensitization Inventory (CSI) in a Pooled Multi-Country Sample. J Pain 19, 317-329

D'Aubigne RM, Postel M (1954): Functional results of hip arthroplasty with acrylic prosthesis. J Bone Joint Surg 36-A, 451-475

de Kruijf M, Peters MJ, C. Jacobs L, Tiemeier H, Nijsten T, Hofman A, Uitterlinden AG, Huygen FJPM, van Meurs JBJ (2015): Determinants for Quantitative Sensory Testing and the Association with Chronic Musculoskeletal Pain in the General Elderly Population. Pain Pract $16,831-841$

Dhondt W, Willaeys T, Verbruggen LA, Oostendorp RA, Duquet W (1999): Pain threshold in patients with rheumatoid arthritis and effect of manual oscillations. Scand J Rheumatol 28, 88-93

D’Mello R, Dickenson AH (2008): Spinal cord mechanisms of pain. Br J Anaesth 101, 8-16

Duncan R, Peat G, Thomas E, Hay E, McCall I, Croft P (2007): Symptoms and radiographic osteoarthritis: not as discordant as they are made out to be? Ann Rheum Dis 66, 86-91

Dunlop DD, Manheim LM, Yelin EH, Song J, Chang RW (2003): The costs of arthritis. Arthritis Care Res 49, 101-113

Dye SF, Vaupel GL, Dye CC (1998): Conscious Neurosensory Mapping of the Internal Structures of the human knee without intraarticular anesthesia. Am J Sports Med 26, 773-777

Ellert U, Kurth BM (2013): Health related quality of life in adults in Germany: results of the German Health Interview and Examination Survey for Adults (DEGS1) Bundesgesundheitsbl Gesundheitsforsch, Gesundheitsschutz 56, 643-649

Emery CF, Keefe FJ, France CR, Affleck G, Waters S, Fondow MDM, McKee DC, France JL, Hackshaw KV, Caldwell DS, Stainbrook D (2006): Effects of a Brief Coping Skills Training Intervention on Nociceptive Flexion Reflex Threshold in Patients Having Osteoarthritic Knee Pain: A Preliminary Laboratory Study of Sex Differences. J Pain Symptom Manage 31, 262-269

Eriksen JR, Poornoroozy P, Jørgensen LN, Jacobsen B, Friis-Andersen HU, Rosenberg J (2009): Pain, quality of life and recovery after laparoscopic ventral hernia repair. Hernia J Hernias Abdom Wall Surg 13, 13-21

Erlenwein J, Müller M, Falla D, Przemeck M, Pfingsten M, Budde S, Quintel M, Petzke F (2017): Clinical relevance of persistent postoperative pain after total hip replacement - a prospective observational cohort study. J Pain Res 10, 2183-2193

Falla D, Hodges PW (2017): Individualized Exercise Interventions for Spinal Pain. Exerc Sport Sci Rev 45, 105-115 
Felson DT, Chaisson CE, Hill CL, Totterman SM, Gale ME, Skinner KM, Kazis L, Gale DR (2001): The association of bone marrow lesions with pain in knee osteoarthritis. Ann Intern Med 134, 541-549

Fillingim RB, King CD, Ribeiro-Dasilva MC, Rahim-Williams B, Riley JL (2009): Sex, Gender, and Pain: A Review of Recent Clinical and Experimental Findings. J Pain 10, 447-485

Finan PH, Buenaver LF, Bounds SC, Hussain S, Park RJ, Haque UJ, Campbell CM, Haythornthwaite JA, Edwards RR, Smith MT (2013): Discordance Between Pain and Radiographic Severity in Knee Osteoarthritis. Arthritis Rheum 65

Fischer AA (1987): Pressure algometry over normal muscles. Standard values, validity and reproducibility of pressure threshold. Pain 30, 115-126

Fontana A, Bistolfi A, Crova M, Rosso F, Massazza G (2012): Arthroscopic Treatment of Hip Chondral Defects: Autologous Chondrocyte Transplantation Versus Simple Debridement-A Pilot Study. Arthroscopy 28, 322-329

Freynhagen R, Baron R, Gockel U, Tölle TR (2006): painDETECT: a new screening questionnaire to identify neuropathic components in patients with back pain. Curr Med Res Opin 22, 1911-1920

Freynhagen R, Tölle TR, Gockel U, Baron R (2016): The painDETECT project - far more than a screening tool on neuropathic pain. Curr Med Res Opin 1-109

Fruhstorfer H, Lindblom U, Schmidt WC (1976): Method for quantitative estimation of thermal thresholds in patients. J Neurol Neurosurg Psychiatry 39, 1071-1075

Gasser H (1941): The Classification of Nerve Fibers. Ohio J of Science 41, 145-159

George SZ, Bishop MD, Bialosky JE, Zeppieri G, Robinson ME (2006): Immediate effects of spinal manipulation on thermal pain sensitivity: an experimental study. BMC Musculoskelet Disord 7, 68

Gerbershagen HJ, Ozgür E, Straub K, Dagtekin O, Gerbershagen K, Petzke F, Heidenreich A, Lehmann KA, Sabatowski R (2008): Prevalence, severity, and chronicity of pain and general health-related quality of life in patients with localized prostate cancer. Eur J Pain 12, 339-350

Gerbershagen HJ, Pogatzki-Zahn E, Aduckathil S, Peelen LM, Kappen TH, Wijck AJM van, Kalkman CJ, Meissner W (2014): Procedure-specific Risk Factor Analysis for the Development of Severe Postoperative Pain. Anesthesiol J 120, 1237-1245

Gerecz-Simon EM, Tunks ER, Heale J-A, Kean WF, Buchanan WW (1989): Measurement of pain threshold in patients with rheumatoid arthritis, osteoarthritis, ankylosing spondylitis, and healthy controls. Clin Rheumatol 8, 467-474

Giesecke T, Gracely RH, Williams DA, Geisser ME, Petzke FW, Clauw DJ (2005): The relationship between depression, clinical pain, and experimental pain in a chronic pain cohort. Arthritis Rheum 52, 1577-1584 
Goulston LM, Kiran A, Javaid MK, Soni A, White KM, Hart DJ, Spector TD, Arden NK (2011): Does obesity predict knee pain over fourteen years in women, independently of radiographic changes? Arthritis Care Res 63, 1398-1406

Gracely RH, Petzke F, Wolf JM, Clauw DJ (2002): Functional magnetic resonance imaging evidence of augmented pain processing in fibromyalgia. Arthritis Rheum 46, 1333-1343

Grafe P (2008): Nozizeption und Schmerz. In: Speckmann E-J, Hescheler J, Köhling R: Physiologie 5. Auflage Urban und Fischer/Elsevier Verlag, München/Jena 2008, 68-80

Granges G, Littlejohn G (1993): Pressure pain threshold in pain-free subjects, in patients with chronic regional pain syndromes, and in patients with fibromyalgia syndrome. Arthritis Rheum 36, 642-646

Graven-Nielsen T, Wodehouse T, Langford RM, Arendt-Nielsen L, Kidd BL (2012): Normalization of widespread hyperesthesia and facilitated spatial summation of deep-tissue pain in knee osteoarthritis patients after knee replacement. Arthritis Rheum 64, 2907-2916

Grönblad M, Liesi P, Korkala O, Karaharju E, Polak J (1984): Innervation of human bone periosteum by peptidergic nerves. Anat Rec 209, 297-299

Gupta A, Silman AJ, Ray D, Morriss R, Dickens C, MacFarlane GJ, Chiu YH, Nicholl B, McBeth J (2007): The role of psychosocial factors in predicting the onset of chronic widespread pain: results from a prospective population-based study. Rheumatol 46, 666-671

Gureje O, Von Korff M, Kola L, Demyttenaere K, He Y, Posada-Villa J, Lepine JP, Angermeyer MC, Levinson D, De Girolamo G, et al. (2008): The relation between multiple pains and mental disorders: Results from the World Health Surveys. Pain 135, 82-91

Harris EC, Coggon D (2015): Hip Osteoarthritis and work. Clin Rheumatol 29, 462

Häuser W, Jung E, Erbslöh-Möller B, Gesmann M, Kühn-Becker H, Petermann F, Langhorst J, Weiss T, Winkelmann A, Wolfe F (2012): Validation of the Fibromyalgia Survey Questionnaire within a Cross-Sectional Survey. PLOS ONE 7

Häuser W, Neugebauer E, Petzke F (2015): Healthcare services research on pain in Germany : A survey. Schmerz 29, 469-478

Hochman JR, Davis AM, Elkayam J, Gagliese L, Hawker GA (2013): Neuropathic pain symptoms on the modified painDETECT correlate with signs of central sensitization in knee osteoarthritis. Osteoarthritis Cartilage 21, 1236-1242

Hootman JM, Helmick CG (2006): Projections of US prevalence of arthritis and associated activity limitations. Arthritis Rheum 54, 226-229

Horstmann T, Heitkamp H-C, Haupt G, Merk J, Mayer F, Dickhuth H-H (2001): Possibilities and limitations of sports therapy in patients with osteoarthrosis and prothesis of the hip. Sportmed 52, 274-278

Hunt I (1999): The prevalence and associated features of chronic widespread pain in the community using the „Manchester“ definition of chronic widespread pain. Rheumatology 38 , 275-279 
International Association For The Study Of Pain (IASP) - Taxonomy. http://www.iasp-pain.org/Taxonomy; Zugriff am 12.10.2015

Im H-J, Kim J, Li X, Kotwal N, Sumner DR, van Wijnen AJ, Davis FJ, Yan D, Levine B, Henry JL, et al. (2010): Alteration of Sensory Neurons and Spinal Response To An Experimental Osteoarthritis Pain Model. Arthritis Rheum 62, 2995-3005

Jaschinski T, Pieper D, Eikermann M, Steinhausen S, Linke C, Heitmann T, Pani M, Neugebauer E (2014): Aktueller Status der Hüft- und Knieendoprothetik in Deutschland Ergebnisse einer bundesweiten Umfrage. Orthop Unfallchirurgie 152, 455-461

Jinks C, Jordan K, Croft P (2007): Osteoarthritis as a public health problem: the impact of developing knee pain on physical function in adults living in the community: (KNEST 3). Rheumatology 46, 877-881

Karsdal MA, Christiansen C, Ladel C, Henriksen K, Kraus VB, Bay-Jensen AC (2014): Osteoarthritis - a case for personalized health care? Osteoarthritis Cartilage 22, 7-16

Kavchak AJE, Fernández-de-las-Peñas C, Rubin LH, Arendt-Nielsen L, Chmell SJ, Durr RK, Courtney CA (2012): Association Between Altered Somatosensation, Pain, and Knee Stability in Patients With Severe Knee Osteoarthrosis: Clin J Pain 28, 589-594

Kellgren JH, Lawrence JS (1957): Radiological Assessment of Osteo-Arthrosis. Rheum Dis 16, 494-502

Khan AM, McLoughlin E, Giannakas K, Hutchinson C, Andrew JG (2004): Hip osteoarthritis: where is the pain? Ann R Coll Surg Engl 86, 119-121

Kim C, Nevitt MC, Niu J, Clancy MM, Lane NE, Link TM, Vlad S, Tolstykh I, Jungmann PM, Felson DT, Guermazi A (2015): Association of hip pain with radiographic evidence of hip osteoarthritis: diagnostic test study. BMJ 351,

Kirwan JR, Currey HLF, Freeman M, Snow S, Young PJ (1994): Overalllong-term impact of total hip and knee joint replacement surgery on patients with osteoarthritis and rheumatoid arthritis. Rheumatology 33, 357-360

Klasen BW, Hallner D, Schaub C, Willburger R, Hasenbring M (2004): Validation and reliability of the German version of the Chronic Pain Grade questionnaire in primary care back pain patients. GMS Psycho-Soc Med 1, 1-12

Kosek E, Ordeberg G (2000a): Abnormalities of somatosensory perception in patients with painful osteoarthritis normalize following successful treatment. Eur J Pain 4, 229-238

Kosek E, Ordeberg G (2000b): Lack of pressure pain modulation by heterotopic noxious conditioning stimulation in patients with painful osteoarthritis before, but not following, surgical pain relief. Pain $88,69-78$

Lavand'homme P (2017): 'Why me?' The problem of chronic pain after surgery. Br J Pain 11, 162-165

Lee YC, Lu B, Bathon JM, Haythornthwaite JA, Smith MT, Page GG, Edwards RR (2011): Pain Sensitivity and Pain Reactivity in Osteoarthritis. Arthritis Care Res 63, 320-327 
Lluch Girbes E, Nijs J, Torres-Cueco R, Lopez Cubas C (2013): Pain Treatment for Patients With Osteoarthritis and Central Sensitization. Phys Ther 23, 842-851

Locher H: Inhibitorische Systeme. In: Locher H, Casser H-R, Strohmeier M, Grifka J: Spezielle Schmerztherapie der Halte- und Bewegungsorgane. Georg Thieme Verlag, Stuttgart 2011, 61-65

Loeser RF, Goldring SR, Scanzello CR, Goldring MB (2012): Osteoarthritis: A Disease of the Joint as an Organ. Arthritis Rheum 64, 1697

Loggia ML, Berna C, Kim J, Cahalan CM, Martel M-O, Gollub RL, Wasan AD, Napadow V, Edwards RR (2015): The lateral prefrontal cortex mediates the hyperalgesic effects of negative cognitions in chronic pain patients. J Pain 16, 692-699

Louw A, Diener I, Butler DS, Puentedura EJ (2011): The Effect of Neuroscience Education on Pain, Disability, Anxiety, and Stress in Chronic Musculoskeletal Pain. Arch Phys Med Rehabil 22, 2041-2056

Lundblad H, Kreicbergs A, Jansson KA (2008): Prediction of persistent pain after total knee replacement for osteoarthritis. J Bone Joint Surg Br 20, 166-171

Magerl W, Krumova EK, Baron R, Tölle T, Treede R-D, Maier C (2010): Reference data for quantitative sensory testing (QST): Refined stratification for age and a novel method for statistical comparison of group data. Pain 151, 598-605

Malfait AM, Schnitzer TJ (2013): Towards a mechanism-based approach to pain management in osteoarthritis. Nat Rev Rheumatol 2, 654-664

Margolis RB, Tait RC, Krause SJ (1986): A rating system for use with patient pain drawings. Pain 24, 57-65

Mayer TG, Neblett R, Cohen H, Howard KJ, Choi YH, Williams MJ, Perez Y, Gatchel RJ (2012): The Development and Psychometric Validation of the Central Sensitization Inventory. Pain Pract 12, 276-285

Mease PJ, Hanna S, Frakes EP, Altman RD (2011): Pain Mechanisms in Osteoarthritis: Understanding the Role of Central Pain and Current Approaches to Its Treatment. J Rheumatol 38, 1546-1551

Meeus M, Nijs J, Van de Wauwer N, Toeback L, Truijen S (2008): Diffuse noxious inhibitory control is delayed in chronic fatigue syndrome: An experimental study. Pain 139, 439-448

Melzack R, Wall PD (1965): Pain mechanisms: A new theory. Science 150, 971-9

Meßlinger K (2010): Somatoviszerale Sensibilität. In: Klinke R, Silbernagl S, Pape HC, Bauer C, Brenner B, Bruggencate G: Physiologie. 6., vollst. überarb. Auflage; Thieme Verlag, Stuttgart 2010, 644-669

Meyer K, Sprott H, Mannion AF (2008): Cross-cultural adaptation, reliability, and validity of the German version of the Pain Catastrophizing Scale. J Psychosom Res 64, 469-478 
Murray DW, Fitzpatrick R, Rogers K, Pandit H, Beard DJ, Carr AJ, Dawson J (2007): The use of the Oxford hip and knee scores. Bone Jt J 82-B, 1010-1014

Naal FD, Sieverding M, Impellizzeri FM, von Knoch F, Mannion AF, Leunig M (2009): Reliability and Validity of the Cross-Culturally Adapted German Oxford Hip Score. Clin Orthop 467, 952-957

Naal FD, Impellizzeri FM, Lenze U, Wellauer V, Eisenhart-Rothe R von, Leunig M (2015): Clinical improvement and satisfaction after total joint replacement: a prospective 12-month evaluation on the patients' perspective. Qual Life Res 24, 2917-2925

Neogi T, Felson D, Niu J, Nevitt M, Lewis CE, Aliabadi P, Sack B, Torner J, Bradley L, Zhang Y (2009): Association between radiographic features of knee osteoarthritis and pain: results from two cohort studies. BMJ 339, b2844

Neogi T, Li S, Peloquin C, Misra D, Zhang Y (2018): Effect of bisphosphonates on knee replacement surgery. Ann Rheum Dis 77 , 92-97

Nijs J, Torres-Cueco R, van Wilgen CP, Girbes EL, Struyf F, Roussel N, van Oosterwijck J, Daenen L, Kuppens K, Vanwerweeen L, et al. (2014): Applying modern pain neuroscience in clinical practice: criteria for the classification of central sensitization pain. Pain Physician 17, $447-457$

Nilges P, Köster B, Schmidt CO (2007): Pain acceptance - concept and validation of a German version of the chronic pain acceptance questionnaire. Schmerz 21, 57-58

Nilges P, Essau C (2015): Die Depressions-Angst-Stress-Skalen. Schmerz 6, 649-657

O'Driscoll SL, Jayson MIV (1974): Pain Threshold Analysis in Patients with Osteoarthrosis of Hip. Br Med J 3, 714

OECD (2013): Health at a Glance

http://www.oecd.org/publications/health-at-a-glance-2013-health-glance-2013-en.htm; Zugriff am 04.08.2018

O’Leary H, Smart KM, Moloney NA, Doody CM (2016): Nervous System Sensitization as a Predictor of Outcome in the Treatment of Peripheral Musculoskeletal Conditions: A Systematic Review. Pain Pract 17, 249-266

O’Neill S, Manniche C, Graven-Nielsen T, Arendt-Nielsen L (2007): Generalized deep-tissue hyperalgesia in patients with chronic low-back pain. Eur J Pain 11, 415-420

Parks EL, Geha PY, Baliki MN, Katz J, Schnitzer TJ, Apkarian AV (2011): Brain activity for chronic knee osteoarthritis: dissociating evoked pain from spontaneous pain. Eur J Pain 15, 843

Pavlaković G, Petzke F (2010): The role of quantitative sensory testing in the evaluation of musculoskeletal pain conditions. Curr Rheumatol Rep 12, 455-461

Peeters G, Edwards KL, Brown WJ, Barker AL, Arden N, Redmond AC, Conaghan PG, Cicuttini F, Mishra GD (2017): Potential effect modifiers of the association between physical activity patterns and joint symptoms in middle aged women. Arthritis Care Res I, 1012-1021 
Perkins FM, Kehlet H (2000): Chronic Pain as an Outcome of Surgery, A Review of Predictive Factors. Anesthesiol J 23, 1123-1133

Petzke F, Harris RE, Williams DA, Clauw DJ, Gracely RH (2005): Differences in unpleasantness induced by experimental pressure pain between patients with fibromyalgia and healthy controls. Eur J Pain 2, 325-335

Pfau DB, Rolke R, Nickel R, Treede R-D, Daublaender M (2009): Somatosensory profiles in subgroups of patients with myogenic temporomandibular disorders and Fibromyalgia Syndrome. Pain 147, 72-83

Pfau DB, Geber C, Birklein F, Treede R-D (2012): Quantitative sensory testing of neuropathic pain patients: potential mechanistic and therapeutic implications. Curr Pain Headache Rep 16, 199-206

Pfingsten M, Leibing E, Harter W, Kröner-Herwig B, Hempel D, Kronshage U, Hildebrandt J (2001): Fear-avoidance behavior and anticipation of pain in patients with chronic low back pain: a randomized controlled study. Pain Med 2, 259-266

Pfingsten M, Nagel B, Emrich O, Seemann H, Lindena G (2006): Handbuch Deutscher Schmerz-Fragebogen. Deutsche Schmerzgesellschaft e.V., Berlin 2006

Pfingsten M, Flor H, Nilges P (2015): Psychological approaches to pain in Germany : Review and outlook. Schmerz 29, 544-549

Quintana JM, Arostegui I, Escobar A, Azkarate J, Goenaga JI, Lafuente I (2008): Prevalence of knee and hip osteoarthritis and the appropriateness of joint replacement in an older population. Arch Intern Med 168, 1576-1584

Rabenberg M (2013): Gesundheitsberichterstattung des Bundes: Arthrose. Hrsg. Robert KochInstitut, Berlin 2013, Heft 54

Rehart S, Lehnert H (2008): Aktuelle Aspekte zur Arthrose. Rheumatol 67, 305-314

Reyes C, Leyland KM, Peat G, Cooper C, Arden NK, Prieto-Alhambra D (2016): Association between overweight and obesity and risk of clinically diagnosed knee, hip, and hand osteoarthritis: a population-based cohort study. Arthritis Rheumatol 68, 1869

Rice D, Mehta S, Shapiro A, Pope J, Harth M, Morley-Forster P, Sequeira K, Teasell R (2016): Psychological Distress in Out-Patients Assessed for Chronic Pain Compared to Those with Rheumatoid Arthritis. Pain Res Manag 2016, 1-7

Riley JL, Robinson ME, Wise EA, Myers CD, Fillingim RB (1998): Sex differences in the perception of noxious experimental stimuli: a meta-analysis. Pain 74, 181-187

Ritter MA, Carr K, Herbst SA, Eizember LE, Keating EM, Faris PM, Meding JB (1996): Outcome of the contralateral hip following total hip arthroplasty for osteoarthritis. J Arthroplasty 11, 242-246

Rolke R, Andrews Campbell K, Magerl W, Treede R-D (2005): Deep pain thresholds in the distal limbs of healthy human subjects. Eur J Pain 2, 39-48 
Rolke R, Magerl W, Campbell KA, Schalber C, Caspari S, Birklein F, Treede R-D (2006a): Quantitative sensory testing: a comprehensive protocol for clinical trials. Eur J Pain 10, 77-77

Rolke R, Baron R, Maier C, Tölle TR, Treede R-D, Beyer A, Binder A, Birbaumer N, Birklein F, Bötefür IC, et al. (2006b): Quantitative sensory testing in the German Research Network on Neuropathic Pain (DFNS): standardized protocol and reference values. Pain 123, 231-243

Rusu AC, Kreddig N, Hallner D, Hülsebusch J, Hasenbring MI (2014): Fear of movement/(Re)injury in low back pain: confirmatory validation of a German version of the Tampa Scale for Kinesiophobia. Musculoskelet Disord 15, 280

Salaffi F, Carotti M, Stancati A, Grassi W (2005): Health-related quality of life in older adults with symptomatic hip and knee osteoarthritis: a comparison with matched healthy controls. Aging Clin Exp Res 17, 255-263

Sandkühler J (2000): Learning and memory in pain pathways. Pain 88, 113-118

Sandrini G, Serrao M, Rossi P, Romaniello A, Cruccu G, Willer JC (2005): The lower limb flexion reflex in humans. Prog Neurobiol 77, 353-395

Sanzarello I, Merlini L, Rosa MA, Perrone M, Frugiuele J, Borghi R, Faldini C (2016): Central sensitization in chronic low back pain: A narrative review. J Back Musculoskelet Rehabil 22, 625-633

Schäfer AGM, Joos LJ, Roggemann K, Waldvogel-Röcker K, Pfingsten M, Petzke F (2017): Pain experiences of patients with musculoskeletal pain + central sensitization: A comparative Group Delphi Study. PLoS ONE 12

Seminowicz DA, Shpaner M, Keaser ML, Krauthamer GM, Mantegna J, Dumas JA, Newhouse PA, Filippi C, Keefe FJ, Naylor MR (2013): Cognitive behavioral therapy increases prefrontal cortex gray matter in patients with chronic pain. J Pain 14, 1573-1584

Shpaner M, Kelly C, Lieberman G, Perelman H, Davis M, Keefe FJ, Naylor MR (2014): Unlearning chronic pain: A randomized controlled trial to investigate changes in intrinsic brain connectivity following Cognitive Behavioral Therapy. NeuroImage Clin 5, 365-376

Sluka KA, Berkley KJ, O'Connor MI, Nicolella DP, Enoka RM, Boyan BD, Hart DA, Resnick E, Kwoh CK, Tosi LL, et al. (2012): Neural and psychosocial contributions to sex differences in knee osteoarthritic pain. Biol Sex Differ 3, 26

Smart KM, Blake C, Staines A, Doody C (2010a): Clinical indicators of „nociceptive“, „peripheral neuropathic“ and „central“ mechanisms of musculoskeletal pain. A Delphi survey of expert clinicians. Man Ther 15, 80-87

Smart KM, Curley A, Blake C, Staines A, Doody C (2010b): The reliability of clinical judgments and criteria associated with mechanisms-based classifications of pain in patients with low back pain disorders: a preliminary reliability study. J Man Manip Ther 18, 102-110

Smith MD, Triantafillou S, Parker A, Youssef PP, Coleman M (1997): Synovial membrane inflammation and cytokine production in patients with early osteoarthritis. J Rheumatol 24, $365-371$ 
Smith MT, Finan PH, Buenaver LF, Robinson M, Haque U, Quain A, McInrue E, Han D, Leoutsakis J, Haythornthwaite JA (2015): Cognitive-Behavioral Therapy for Insomnia in Knee Osteoarthritis: A Randomized, Double-Blind, Active Placebo-Controlled Clinical Trial. Arthritis Rheumatol 67, 1221-1233

Staud R (2011): Evidence for Shared Pain Mechanisms in Osteoarthritis, Low Back Pain, and Fibromyalgia. Curr Rheumatol Rep 13, 513

Stucki G, Meier D, Stucki S, Michel BA, Tyndall AG, Dick W, Theiler R (1996): Evaluation of a German version of WOMAC (Western Ontario and McMaster Universities) Arthrosis Index. Rheumatol 55, 40-49

Suokas AK, Walsh DA, McWilliams DF, Condon L, Moreton B, Wylde V, Arendt-Nielsen L, Zhang W (2012): Quantitative sensory testing in painful osteoarthritis: a systematic review and meta-analysis. Osteoarthritis Cartilage 20, 1075-1085

Tesarz J, Eich W, Treede R, Gerhardt A (2016): Altered pressure pain thresholds and increased wind-up in adult patients with chronic back pain with a history of childhood maltreatment: a quantitative sensory testing study. Pain 157, 1799-1809

Traue H C, Horn A B, Kessler H, Jerg-Bretzke L (2013): Psyche und Schmerz. In: Baron R, Koppert W, Strumpf M, Willweber-Strumpf A: Praktische Schmerztherapie. 3. Auflage; Springer Medizin Verlag, Heidelberg 2013, 117-128

Treede R-D (2013a): Quantitativ-sensorische Testung. In: Baron R, Koppert W, Strumpf M, Willweber-Strumpf A: Praktische Schmerztherapie. 3. Auflage; Springer Medizin Verlag, Heidelberg 2013, 87-94

Treede, R-D (2013b): Entstehung der Schmerzchronifizierung. In: Baron R, Koppert W, Strumpf M, Willweber-Strumpf A: Praktische Schmerztherapie. 3. Auflage; Springer Medizin Verlag, Heidelberg 2013, 3-13

Treede R-D, Rief W, Barke A, Aziz Q, Bennett MI, Benoliel R, Cohen M, Evers S, Finnerup NB, First MB, et al. (2015): A classification of chronic pain for ICD-11. Pain 156, 1003-1007

Van Hecke O, Kamerman PR, Attal N, Baron R, Bjornsdottir G, Bennett DLH, Bennett MI, Bouhassira D, Diatchenko L, Freeman R, et al. (2015): Neuropathic pain phenotyping by international consensus (NeuroPPIC) for genetic studies: a NeuPSIG systematic review, Delphi survey, and expert panel recommendations. Pain 156, 2337-2353

Verdugo R, Ochoa JL (1992): Quantitative Somatosensory Thermotest: A Key Method for Functional Evaluation of Small Calibre Afferent Channels. Brain 115, 893-913

Von Dincklage F, Send K, Hackbarth M, Rehberg B, Baars JH (2009a): Comparison of the nociceptive flexion reflex threshold and the bispectral index as monitors of movement responses to noxious stimuli under propofol mono-anaesthesia. Br J Anaesth 102, 244-250 Von Dincklage F, Hackbarth M, Schneider M, Baars JH, Rehberg B (2009b): Introduction of a continual RIII reflex threshold tracking algorithm. Brain Res 1260, 24-29

Von Korff M, Ormel J, Keefe FJ, Dworkin SF (1992): Grading the severity of chronic pain. Pain 50, 133-149 
Von Korff M, Miglioretti D (2005): A prognostic approach to defining chronic pain. Pain 117, 304-313

Vonsy JL, Ghandehari J, Dickenson AH (2009): Differential analgesic effects of morphine and gabapentin on behavioural measures of pain and disability in a model of osteoarthritis pain in rats. Eur J Pain 13, 786-793

Wengler A, Nimptsch U, Mansky T (2014): Hip and Knee Replacement in Germany and the USA: Analysis of Individual Inpatient Data From German and US Hospitals for the Years 2005 to 2011. Dtsch Ärztebl Int 111, 407

WMA: World Medical Association General Assembly (2013): Declaration of Helsinki. Ethical Principles for Medical Research Involving Human Subjects. Fortaleza, Brazil. http://www.wma.net/en/30publications/10policies/b3/; Zugriff am 23.10.2015

Wolff R, Clar C, Lerch C, Kleijnen J (2011): Epidemiologie von nicht tumorbedingten chronischen Schmerzen in Deutschland. Schmerz 25, 26-44

Woolf CJ (2011): Central sensitization: Implications for the diagnosis and treatment of pain. Pain 152, S2-15

Wylde V, Blom AW, Whitehouse SL, Taylor AH, Pattison GT, Bannister GC (2009): PatientReported Outcomes After Total Hip and Knee Arthroplasty: Comparison of Midterm Results. J Arthroplasty 24, 210-216

Wylde V, Sayers A, Odutola A, Gooberman-Hill R, Dieppe P, Blom A w. (2017): Central sensitization as a determinant of patients' benefit from total hip and knee replacement. Eur J Pain 21, 357-365

Ziegler EA, Magerl W, Meyer RA, Treede R-D (1999): Secondary hyperalgesia to punctate mechanical stimuliCentral sensitization to A-fibre nociceptor input. Brain 122, 2245-2257 